\title{
Biomedical Optical Imaging
}

\author{
Academic Staff \\ Professor James G. Fujimoto \\ Research Staff, Visiting Scientists and Visiting Students \\ Dr. Bernhard Baumann, Martin Kraus, Dr. Ben Potsaid, Dr. Chao Zhou

\section{Scientific Collaborators} \\ Dr. Jay S. Duker, M.D., Varsha Manjunath, Lauren Branchini (New England Eye Center) \\ Dr. Joel Schuman, M.D. (University of Pittsburgh Medical Center) \\ Dr. Edward Feener, Dr. Allen Clermont (Joslin Diabetes Center) \\ Dr. Hiroshi Mashimo, M.D., Ph.D. (VA Medical Center) \\ Dr. Joseph Schmitt, Dr. Desmond Adler (LightLab Imaging) \\ Dr. David A. Boas, Lana Ruvinskaya, Dr. Anna Devor, Dr. Vivek J. Srinivasan (MGH) \\ Alex Cable, Dr. James Jiang, Dr. Ben Potsaid (Thorlabs, Inc.)

\section{Graduate Students} \\ Woo Jhon Choi, Hsiang-Chieh Lee, Jonathan J. Liu, Tsung-Han Tsai \\ Technical and Support Staff \\ Dorothy A. Fleischer, Donna L. Gale
}

\section{Research Areas and Projects}

1. Optical coherence tomography (OCT) technology

1.1 Overview of Optical Coherence Tomography

1.2 Spectral / Fourier domain OCT Imaging

1.3 Swept source / Fourier domain OCT Imaging using Swept Lasers

2. OCT in Ophthalmology

2.1 OCT in Ophthalmology

2.2 Ultrahigh Speed Spectral / Fourier Domain OCT Retinal Imaging

2.3 Swept Source / Fourier domain OCT Imaging at $1050 \mathrm{~nm}$ Wavelengths

2.4 Doppler OCT Imaging

2.5 Small Animal Retinal Imaging

2.6 Clinical OCT Studies

3. Motion Correction and Image Enhancement

4. High Speed Three-Dimensional OCT Endoscopic Imaging

3.1 OCT Imaging of the GI Tract

3.2 Assessment of Ablation Therapies in the GI Tract

5. Optical Coherence Tomography and Microscopy for Imaging in the Pathology Laboratory

4.1 Integrated OCT and OCM Imaging of Human Thyroid Pathology

4.2 Integrated OCT and OCM Imaging of Breast Pathology

4.3 Integrated OCT and OCM Imaging of Human Kidney Pathology

6. Functional Brain Imaging with OCT

7. Photothermal modulation for functional OCT 


\section{Optical Coherence tomography (OCT) Technology}

\section{Sponsors}

National Institutes of Health - R01-CA075289-12, R01-EY11289-24

Air Force Office of Scientific Research - FA9550-040-1-0011, FA9550-040-1-0046

Center for Integration of Medicine and Innovation Technology (CIMIT)

\section{Project Staff}

Prof. James G. Fujimoto, Dr. Bernhard Baumann, Dr. Ben Potsaid, Dr. Chao Zhou, WooJhon Choi, Martin Kraus, Hsiang-Chieh Lee, Jonathan J. Liu, Tsung-Han Tsai

Optical coherence tomography (OCT) is an emerging medical imaging and diagnostic technology developed by our research group and collaborators in 1991 [1]. OCT is analogous to ultrasound, measuring the intensity of backreflected or backscattered infrared light, rather than acoustical waves. Figure 1.1 shows a schematic of how OCT images are generated. OCT can perform cross sectional and three dimensional, micron scale imaging of tissue structure. OCT is attractive for biomedical research and clinical imaging for several reasons. Imaging can be performed in real time, allowing tissue microstructure to be visualized without the need to excise and process specimens as in conventional excisional biopsy and histopathology. Image resolutions are 1 to 15 microns, enabling visualization of tissue architectural morphology. OCT can be performed with a wide range of instruments including ophthalmoscopes, small endoscopes, catheters, probes, needles, or surgical instruments. OCT has had a dramatic impact in ophthalmology, where it has become a standard diagnostic for retinal disease and glaucoma [2]. In addition, OCT is an emerging technology for intravascular imaging, where it can identify unstable plaques that are prone to rupture and guide treatment [3]. OCT research combines multiple technologies including photonics, high speed electronics and signal processing, imaging processing, medical device development, biomedical engineering, biomedical research and clinical studies [4]. Our group and collaborators have a vertically integrate research program which combines fundamental science, design and engineering in order to develop new technology for biomedical and clinical studies.

$1 \mathrm{D}$

Axial (Z) Scanning
2 D

Axial (Z) Scanning Transverse (X) Scanning
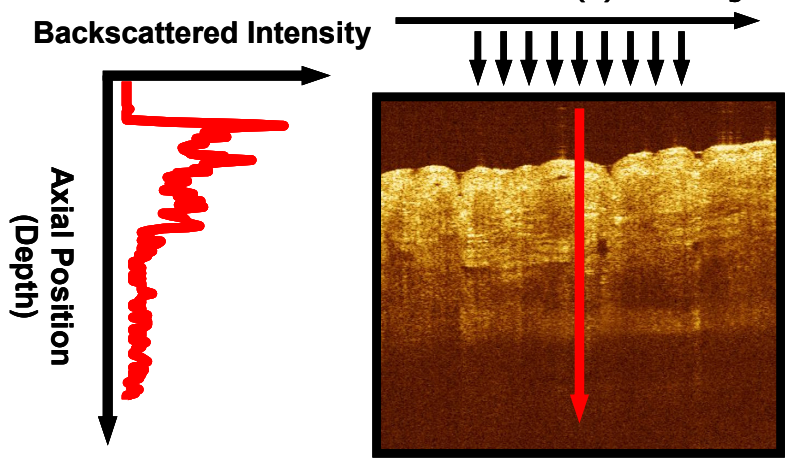

3D

Axial (Z) Scanning XY Scanning

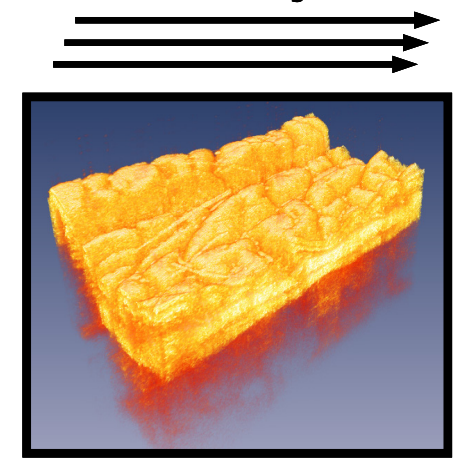

Figure 1.1. Principles of OCT imaging. OCT measures the magnitude and echo time delay of light to generate cross-sectional and three-dimensional images of tissue microstructure and architectural morphology in vivo and in real time. 


\subsection{Overview of Optical Coherence Tomography}

OCT images the internal structure in biological tissue or materials by measuring the magnitude and echo time delay of backreflected or backscattered light. OCT measures echoes of light using interferometry. In general, an OCT system is composed of a light source, an interferometer with a reference mirror and a sample beam path, and a detector, as shown in Figure 1.2. Early OCT systems detected echoes of light based on low coherence interferometry with time domain detection techniques. A low coherence light source is directed into an interferometer and split into a signal and reference beam. Interference between the two beams will only occur when the signal and reference path lengths are matched to within a coherence length of the light. Echoes of light from a sample can be measured by interfering the backreflected or backscattered beam with the reference light, while scanning the reference path length. The interference output of the interferometer is detected as a function of time and demodulated to generate an axial scan. This method detects echoes of light sequentially as the reference path is scanned. The axial scan is a measurement of the light signal versus depth or echo time delay. Cross sectional or volumetric images are generated by scanning the OCT beam in the transverse direction and acquiring multiple axial scan measurements as shown in Figure 1.1.

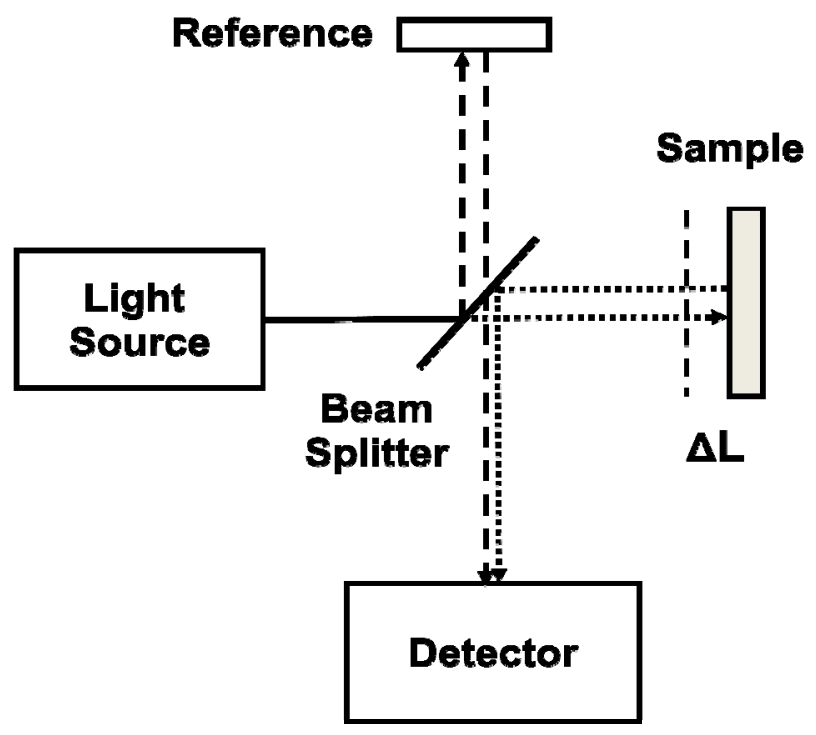

Figure 1.2. Schematic of OCT system. Echoes of light are measured using interferometry. The system consists of a light source, interferometer with sample and reference paths, and a detector.

\subsection{Spectral / Fourier Domain OCT Imaging}

Recent advances in OCT technology have achieved dramatic increases in performance. These new techniques measure the echo time delay of light by Fourier transforming the interference spectrum of the light signal and are therefore known as Fourier domain OCT detection. Different echo time delays of light produce different frequencies of fringes in the interference spectrum. Fourier domain detection techniques with spectrometer based systems (spectral / Fourier domain OCT) or frequency swept laser based systems (swept source / Fourier domain OCT) enable OCT imaging to be performed with unprecedented sensitivities and speeds [5-8]. High speed enables three dimensional OCT imaging (3D-OCT), generating volumetric data sets which contain comprehensive structural information. High speeds also enable the rapid survey of large areas of tissue as well as increased imaging throughput. In addition, high speeds allow multi-frame averaging as well as the acquisition of high-pixel-density images, which can be used to improve image quality or reduce speckle. Fourier domain OCT detection also has the advantage of providing direct access to the interference spectrum, enabling a wide range of phase sensitive applications such as Doppler imaging of blood flow [9-12]. Finally, Fourier domain OCT techniques are also well-suited for numerical dispersion compensation, supporting broad bandwidths and enabling ultrahigh axial image resolutions $[13,14]$. 
Spectral / Fourier domain detection techniques measure the echo time delay of light by using an interferometer with a broadband light source and a spectrometer with high speed linescan camera. Figure 1.3 shows the schematic diagram of a spectral / Fourier-domain OCT system. A broadband light source is coupled into an interferometer with a sample and a reference arm. Backscattered or backreflected light from the sample interferes with light from the reference arm and is detected by the spectrometer and line scan camera. The spectrum at the interferometer output is measured and processed. Light echoes from the sample at different delays generate oscillations or fringes in the interference spectrum, as shown in Figure 1.3B. An increase in delay results in higher oscillation frequency in the interference spectrum. The magnitude and delay of the backscattered light can be measured by Fourier transforming the interference spectrum, as shown in Figure 1.3C.

A

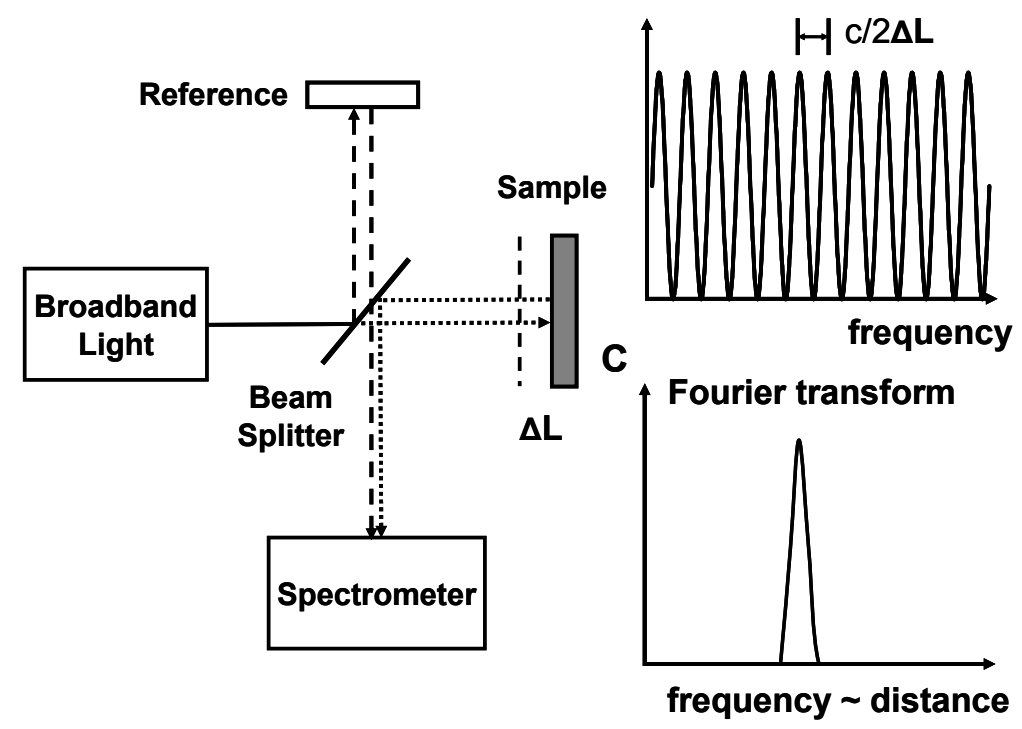

Figure 1.3. Schematic showing spectral / Fourier domain OCT detection. (A) A broadband light source is directed to a Michelson interferometer with a sample and a reference arm. (B) Light from the sample interferes with the reference light and the interference spectrum is recorded by a spectrometer. (C) Light echoes from different delays produce interference in the spectral or Fourier domain. The magnitude and time delay of the light echoes is measured by Fourier transforming the interference spectrum to obtain an axial scan.

Spectral / Fourier domain detection achieves a dramatic increase in sensitivity because it can measure all of the echoes of light from different depths simultaneously [5-7]. In both time domain and Fourier domain detection, the sensitivity is proportional to the exposure time or inversely proportional to acquisition speed. With spectral / Fourier domain detection, the exposure time is approximately the axial scan time. However, with time domain OCT, during the same axial scan time, the reference path must be scanned over the axial depth. This required $M$ different measurements, where $M$ is the number of resolvable elements in one axial scan. This means that for a given axial scan time, the exposure time in time domain detection is decreased by a factor of $M$. Depending on the axial resolution and scan depth, the number of pixels (or resolvable points) in an axial scan is typically 100 to 1000. Therefore, spectral / Fourier domain detection typically provides a 20 to $30 \mathrm{~dB}$ increase in sensitivity compared with time domain detection. Furthermore, since image acquisition speed trades off against sensitivity and the incident power is limited by safety considerations, this enables a 100 to 1000 times increase in imaging speed. 


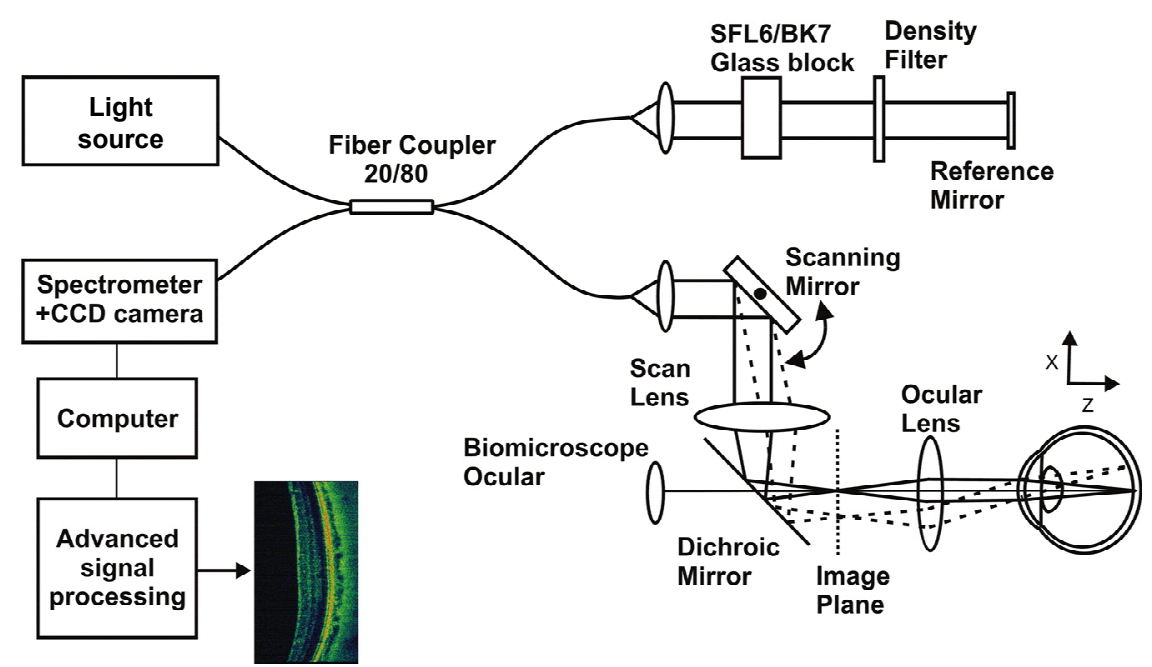

Figure 1.4. Schematic diagram of a high speed ophthalmic OCT system using spectral / Fourier domain detection.

Figure 1.4 shows a schematic of a typical high speed, ultrahigh resolution spectral / Fourier domain OCT system. The light source can be either a femtosecond laser or a broadband superluminescent diode (SLD). Our earlier studies used a femtosecond Ti:Sapphire laser for ultrahigh-resolution imaging, however modern multiplexed SLD light sources can now generate very broad bandwidths and achieve axial resolutions of 3 to 3.5 um [13]. SLD light sources are attractive because they are relatively inexpensive and significantly simpler to operate than femtosecond lasers, enabling easier operation in the clinic. The interferometer is implemented using a fiber optical coupler with the sample arm going to a patient interface. The patient interface consists of two galvanometer-controlled steering mirrors which scan the angle of the OCT beam. The beam is relay imaged onto the retina such that it pivots about the pupil of the eye as the beam is scanned. The scan pattern is controlled by computer. The transverse image resolution is determined by the transverse spot size on the retina and is typically $\sim 20 \mathrm{um}$. The incident optical power on the eye is $750 \mathrm{uW}$, the same exposure used in commercial ophthalmic OCT systems and consistent with the ANSI safety standards. The light backscattered or backreflected from the retina and the light reflected by the reference mirror is combined by the coupler to create an interference spectrum that is detected by the spectrometer. The spectrometer uses a high efficiency transmission grating, imaging lens, and high-speed line CCD or CMOS scan camera. The recorded spectral interferometer signal is rescaled from wavelength into frequency (or k-space) and dispersion corrected before being Fourier transformed to create axial scan data and OCT images.

Figure 1.5 shows a comparison of standard 10 um resolution (from a commercial Zeiss StratusOCT instrument) and high-speed, ultrahigh 3.5 um resolution (from our research prototype instrument) images from a 68-year-old woman with a stage 2 full-thickness macular hole. Both the standard resolution (Fig. 1.5b) and the ultrahigh resolution OCT image (Fig. 1.5c) depict a macular hole. However, the highdefinition, ultrahigh resolution OCT image enables better visualization of the intraretinal layers and cysts, as well as the photoreceptor outer segments. Ultrahigh resolution OCT provides detailed information about the photoreceptor layer and its integrity. 

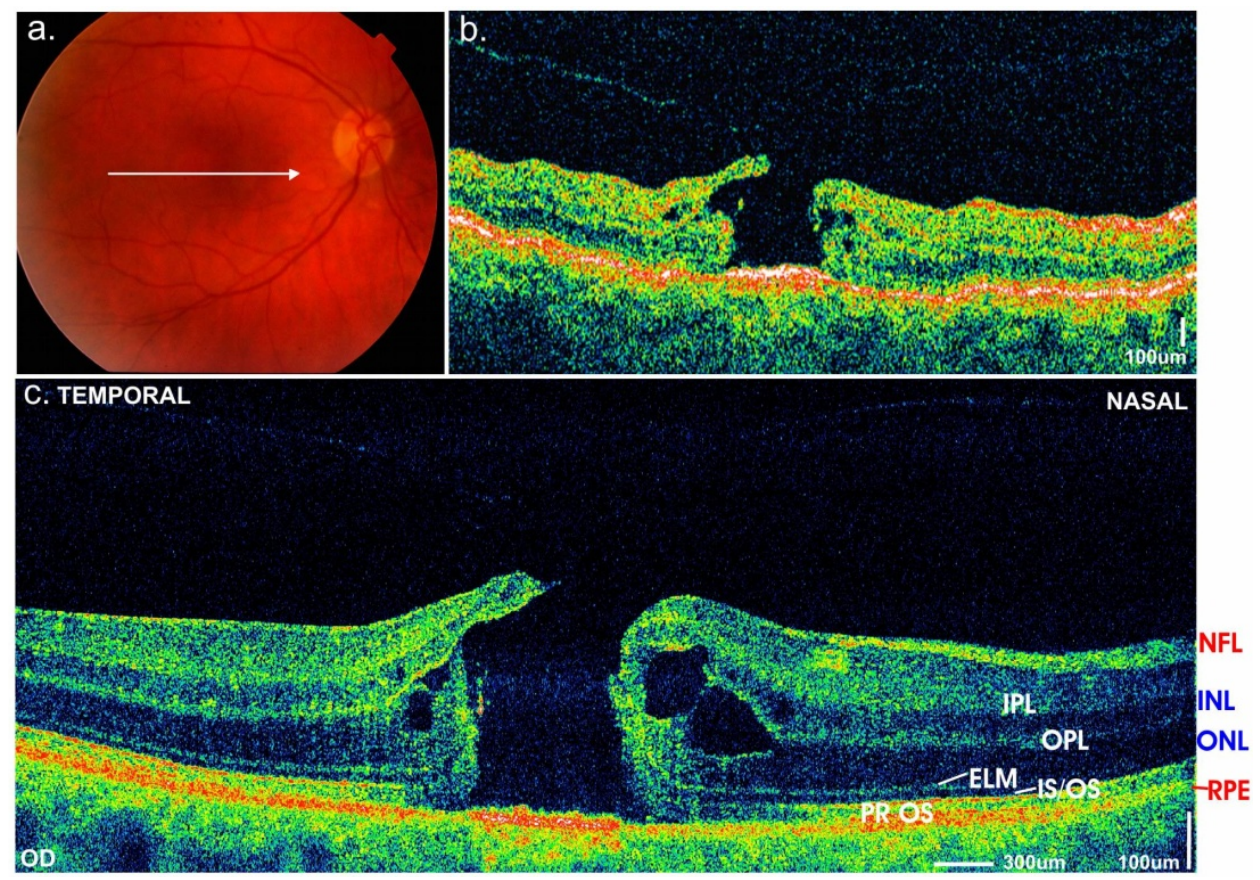

Figure 1.5. High-speed, ultrahigh resolution retinal imaging using spectral / Fourier domain OCT. (A) A 68-year-old woman diagnosed with a stage 2 full-thickness macular hole. (B) OCT image from previous generation commercial Zeiss (Stratus OCT) instrument and (C) high-speed, ultrahighresolution OCT. The image from the spectral / Fourier domain instrument has 3 um axial resolution and is 8192 transverse pixels across. Both images clearly show a macular hole and intraretinal cysts. The high-definition OCT image enables better visualization of cystic changes and photoreceptor impairment. (NFL - nerve fiber layer, IPL - inner plexiform layer, INL - inner nuclear layer, OPL - outer plexiform layer, ONL - outer nuclear layer, ELM - external limiting membrane, IS/OS - photoreceptor inner segment/outer segment junction, PR OS - photoreceptor outer segments, RPE - retinal pigment epithelium)

\subsection{Swept Source / Fourier Domain OCT Imaging using Swept Lasers}

Swept source / Fourier domain OCT is a complementary approach to spectral / Fourier domain OCT. Spectral / Fourier domain OCT uses a broadband light source and a spectrometer to measure interference as a function of wavelength or frequency. In contrast, swept source / Fourier domain OCT uses a swept light source that generates narrow band light whose optical frequency is swept in time. Swept source OCT use a high speed detector and does not require a spectrometer or line scan camera. Figure 1.6 shows a schematic of a swept source / Fourier domain OCT system. Backreflected or backscattered light signals are measured using an interferometer with swept light source. Optical delays produce a beat frequency in the interference signal which is detected and Fourier transformed to measure the optical delay. Swept source OCT has a similar sensitivity advantage to spectral OCT and enables very high imaging speeds.

Swept source OCT has important advantages over spectral / Fourier domain OCT. Swept source OCT uses high speed detectors and does not require a spectrometer or line scan camera. Conventional silicon CCD or CMOS line scan cameras are sensitive only up to $1000 \mathrm{~nm}$ wavelengths. Although InGaAs line scan cameras are available and enable spectral OCT at longer wavelengths, these cameras have limited numbers of pixels and are relatively expensive. Swept source OCT can work at longer wavelengths, such as $1050 \mathrm{~nm}$ or $1300 \mathrm{~nm}$, which are beyond the range of conventional line scan cameras. Imaging at long wavelengths is important for OCT applications in tissues other than the eye, where optical scattering limits image penetration. 
Swept source OCT also has several engineering advantages. Spectrometers have loss, are sensitive to alignment and are bulky. Since swept source OCT uses photo detectors rather than a spectrometer, it can be more compact and have higher sensitivity than spectral / Fourier domain OCT. Since imaging speeds are limited by sensitivity, swept source OCT could ultimately image faster than spectral / Fourier domain OCT. The frequency or wavelength resolution in a swept source system is determined by the laser linewidth and can be significantly better than a spectrometer based spectral / Fourier domain OCT system. This allows imaging to be performed with a greater depth range and higher number of axial pixels than possible with spectral / Fourier domain OCT. Finally, swept source OCT enables rapid imaging of larger fields of view than spectral / Fourier domain OCT because it does not have the fringe wash out problems which occur with rapid beam scanning in spectrometer based detection.

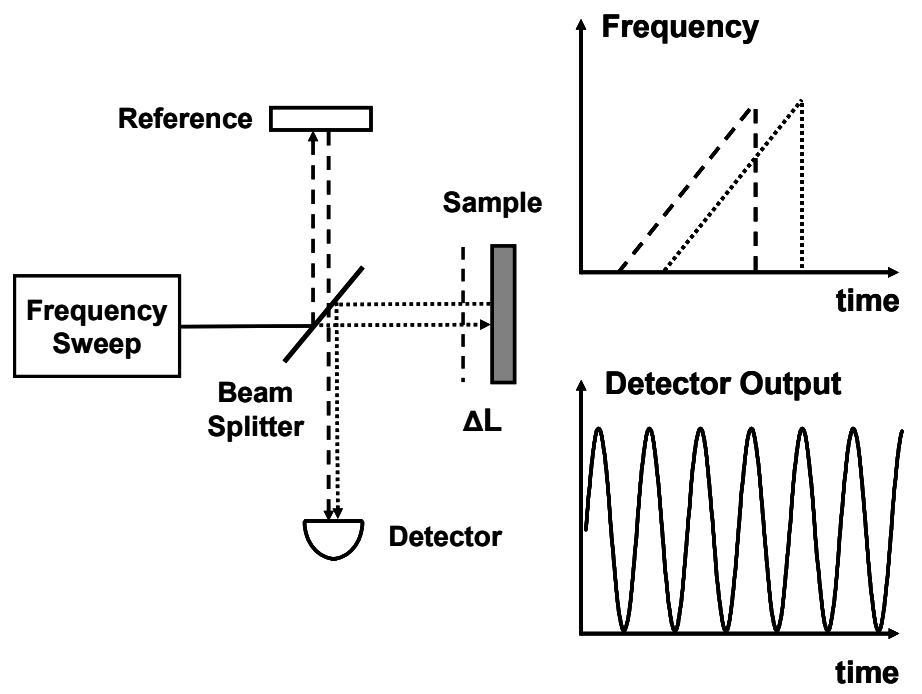

Figure 1.6. Swept source / Fourier domain OCT detection. A frequency swept light source is directed to an interferometer with a sample and reference path. Delayed light from the sample interferes with the reference beam and produces a beat signal which is measured by a high speed detector. The magnitude and time delay of the light can be measured by Fourier transforming the beat signal.

Figure 1.7 shows a schematic of a swept source / Fourier domain OCT instrument. A high efficiency, dual-balanced Michelson interferometer detects the OCT signal. In this configuration, intensity noise from the laser source is cancelled out and the interferometric signal level is doubled by subtracting two out-ofphase interference fringes. The sample beam is scanned over the sample by a two galvanometercontrolled mirrors. A small portion ( $5 \%)$ of the laser light source is coupled to a calibration Mach-Zehnder interferometer in order to measure optical frequency intervals during the laser sweep. This information is used to process the interference fringes so they are uniformly spaced in optical frequency, correcting for nonlinearities in the laser frequency sweep. An A/D card that is synchronized to the laser sweep records the Mach-Zehnder calibration and OCT signals. A computer re-samples the OCT interference fringe signal to be linear in optical frequency, takes the Fourier transform and generates the axial scan.

Swept lasers are a key technology for swept source OCT. The laser sweep repetition rate determines the axial scan repetition rate, the sweep tuning bandwidth determines the axial image resolution and the instantaneous linewidth governs the maximum imaging range. Therefore, swept laser development has become an important area of investigation. Very early studies performed by our group in the 1990s used bulk optics laser designs consisting of a laser cavity and intracavity tuning element, which had slow scan speeds $[15,16]$. Other groups later recognized that swept sources with very high sweep rates were possible. A laser using a fiber coupled semiconductor amplifier gain element and a fiber Fabry-Perot tunable filter within a fiber ring cavity was demonstrated at a sweep rates of $\mathrm{kHz}$ in 2003 [6]. Later, hybrid bulk optic and fiber cavity designs using a fiber-coupled semiconductor amplifier and a tuning element consisting of a diffraction grating and rotating mirror scanner were developed and demonstrated to 
achieve tens to hundreds of $\mathrm{kHz}$ sweep speeds [17-19].

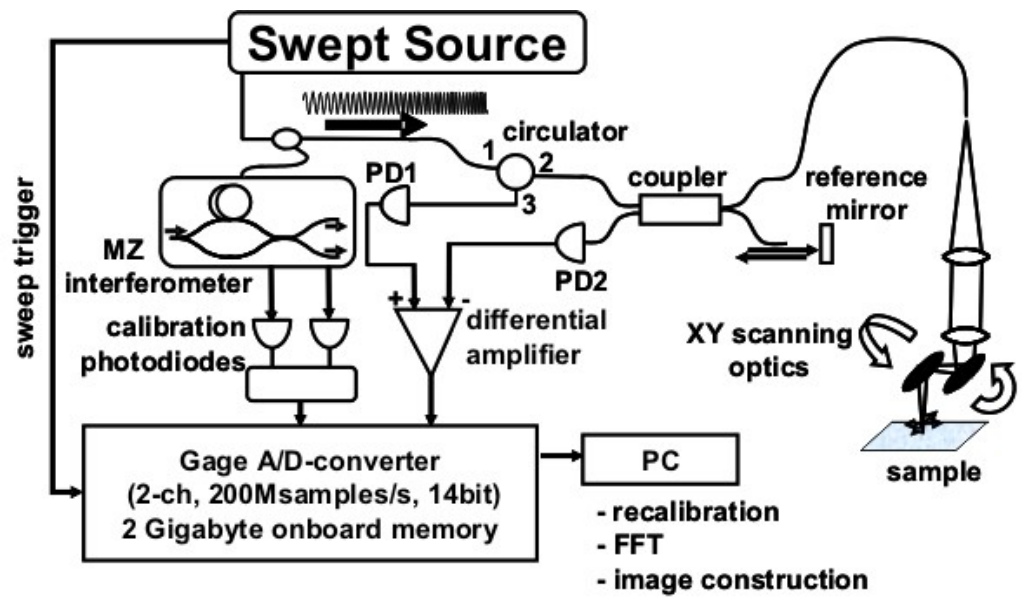

Figure 1.7. Schematic diagram of a swept source / Fourier domain OCT system. The system requires a high speed swept laser and system performance depends directly on the laser performance. Imaging generation requires high speed detection and signal processing. Swept source / Fourier domain OCT utilizes many aspects of photonic communications technologies.

The maximum tuning speed of a laser is limited by the time required to build up lasing from spontaneous emission as the intracavity filter is tuned [20]. In 2005, our group developed a new class of swept laser sources called Fourier-domain modelocked lasers (FDML) [21, 22]. These lasers operate by using a long intracavity fiber delay line and synchronize the sweep repetition rate of the intracavity tuning element, typically an fiber Fabry Perot FFP filter, to the optical roundtrip time of the cavity. Each wavelength in the sweep returns to the filter when it is tuned to the correct wavelength, thus allowing all wavelengths within the sweep to build up and lase simultaneously within the cavity. The FDML laser essentially stores and regenerates the entire sweep in the cavity. This results in an effective quasi-continuous operation and achieves an unprecedented combination of sweep speed, narrow instantaneous linewidth and tuning range. In comparison with traditional swept sources, for FDML operation the lasing does not need to build up from the gain medium each time while the filter is shifting. This non-continuous operation in standard swept lasers forces a tradeoff between sweep speed and other performance characteristics. Axial scan rates can be increased even further by using multiplexing or buffering techniques where laser frequency sweeps are delayed and recombined using optical fiber delays. Using FDML swept lasers and multiplexing techniques, our group achieved axial scan speeds of $370 \mathrm{kHz}$ in research OCT systems [22]. We also demonstrated ultrahigh speed ophthalmic swept source OCT imaging at $1050 \mathrm{~nm}$ [23]. Recently, other groups have used FDML techniques in combination with multiplexing to achieve record axial scan speeds of greater than $1 \mathrm{MHz}$ [24].

Figure 1.8 shows an example of swept source OCT imaging showing 3D-OCT imaging of the Drosophila at 42,000 A-scans per second. Drosophila is a widely accepted model in the study of developmental biology. Due to the high speed of the OCT system, the rapid dynamics of the Drosophila heart could be imaged and assessed. In addition to three-dimensional OCT images, sequential cross-sectional OCT images can be continuously acquired to analyze the structural dynamics of the heart. 


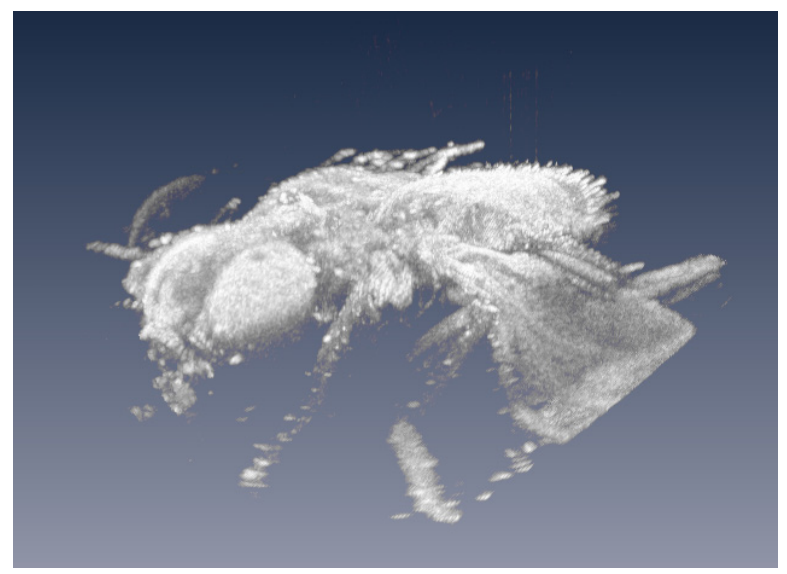

Figure 1.8. 3D-OCT imaging of the Drosophila using a high-speed, swept-source / Fourier domain OCT system.

\section{References}

[1] D. Huang, E. A. Swanson, C. P. Lin, J. S. Schuman, W. G. Stinson, W. Chang, M. R. Hee, T. Flotte, K. Gregory, C. A. Puliafito, and J. G. Fujimoto, "Optical Coherence Tomography," Science, vol. 254, pp. 1178-1181, 1991

[2] J. S. Schuman, C. A. Puliafito, and J. G. Fujimoto, Optical coherence tomography of ocular diseases, 2nd edition. Thorofare, NJ: Slack Inc., 2004.

[3] E. Regar, A. M. G. J. van Leeuwen, and P. W. Serruys, Optical Coherence Tomography in Cardiovascular Research: Taylor and Francis, 2007.

[4] W. Drexler and J. G. Fujimoto, Optical Coherence Tomography Technology and Applications. Berlin Heidelberg New York: Springer, 2008.

[5] R. Leitgeb, C. K. Hitzenberger, and A. F. Fercher, "Performance of Fourier domain vs. time domain optical coherence tomography," Optics Express, vol. 11, pp. 889-894, 2003

[6] M. A. Choma, M. V. Sarunic, C. H. Yang, and J. A. Izatt, "Sensitivity advantage of swept source and Fourier domain optical coherence tomography," Optics Express, vol. 11, pp. 2183-2189, 2003

[7] J. F. de Boer, B. Cense, B. H. Park, M. C. Pierce, G. J. Tearney, and B. E. Bouma, "Improved signal-to-noise ratio in spectral-domain compared with time-domain optical coherence tomography," Optics Letters, vol. 28, pp. 2067-2069, 2003

[8] M. Wojtkowski, T. Bajraszewski, P. Targowski, and A. Kowalczyk, "Real-time in vivo imaging by high-speed spectral optical coherence tomography," Optics Letters, vol. 28, pp. 1745-1747, 2003

[9] R. A. Leitgeb, L. Schmetterer, W. Drexler, A. F. Fercher, R. J. Zawadzki, and T. Bajraszewski, "Real-time assessment of retinal blood flow with ultrafast acquisition by color Doppler Fourier domain optical coherence tomography," Optics Express, vol. 11, pp. 3116-3121, 2003

[10] B. R. White, M. C. Pierce, N. Nassif, B. Cense, B. H. Park, G. J. Tearney, B. E. Bouma, T. C. Chen, and J. F. de Boer, "In vivo dynamic human retinal blood flow imaging using ultra-highspeed spectral domain optical Doppler tomography," Optics Express, vol. 11, pp. 3490-3497, 2003

[11] B. J. Vakoc, S. H. Yun, J. F. de Boer, G. J. Tearney, and B. E. Bouma, "Phase-resolved optical frequency domain imaging," Optics Express, vol. 13, pp. 5483-5493, 2005

[12] S. Makita, Y. Hong, M. Yamanari, T. Yatagai, and Y. Yasuno, "Optical coherence angiography," Optics Express, vol. 14, pp. 7821-7840, 2006

[13] M. Wojtkowski, V. J. Srinivasan, T. H. Ko, J. G. Fujimoto, A. Kowalczyk, and J. S. Duker, "Ultrahigh-resolution, high-speed, Fourier domain optical coherence tomography and methods for dispersion compensation," Optics Express, vol. 12, pp. 2404-2422, 2004

[14] N. Nassif, B. Cense, B. H. Park, S. H. Yun, T. C. Chen, B. E. Bouma, G. J. Tearney, and J. F. de Boer, "In vivo human retinal imaging by ultrahigh-speed spectral domain optical coherence tomography," Opt Lett, vol. 29, pp. 480-2, 2004 
[15] B. Golubovic, B. E. Bouma, G. J. Tearney, and J. G. Fujimoto, "Optical frequency-domain reflectometry using rapid wavelength tuning of a $\mathrm{Cr} 4+$ :forsterite laser," Optics Letters, vol. 22, pp. 1704-1706, 1997

[16] S. R. Chinn, E. A. Swanson, and J. G. Fujimoto, "Optical coherence tomography using a frequency-tunable optical source," Optics Letters, vol. 22, pp. 340-342, 1997

[17] S. H. Yun, G. J. Tearney, B. E. Bouma, B. H. Park, and J. F. de Boer, "High-speed spectraldomain optical coherence tomography at $1.3 \mathrm{mu}$ m wavelength," Optics Express, vol. 11, pp. 3598-3604, 2003

[18] S. H. Yun, G. J. Tearney, J. F. de Boer, N. Iftimia, and B. E. Bouma, "High-speed optical frequency-domain imaging," Optics Express, vol. 11, pp. 2953-2963, 2003

[19] W. Y. Oh, S. H. Yun, G. J. Tearney, and B. E. Bouma, "115 kHz tuning repetition rate ultrahighspeed wavelength-swept semiconductor laser," Optics Letters, vol. 30, pp. 3159-3161, 2005

[20] R. Huber, M. Wojtkowski, K. Taira, J. G. Fujimoto, and K. Hsu, "Amplified, frequency swept lasers for frequency domain reflectometry and OCT imaging: design and scaling principles," Optics Express, vol. 13, pp. 3513-3528, 2005

[21] R. Huber, M. Wojtkowski, and J. G. Fujimoto, "Fourier Domain Mode Locking (FDML): A new laser operating regime and applications for optical coherence tomography," Optics Express, vol. 14, pp. 3225-3237, 2006

[22] R. Huber, D. C. Adler, and J. G. Fujimoto, "Buffered Fourier domain mode locking: unidirectional swept laser sources for optical coherence tomography imaging at 370,000 lines/s," Optics Letters, vol. 31, pp. 2975-2977, 2006

[23] V. J. Srinivasan, D. C. Adler, Y. Chen, I. Gorczynska, R. Huber, J. S. Duker, J. S. Schuman, and J. G. Fujimoto, "Ultrahigh-speed optical coherence tomography for three-dimensional and en face imaging of the retina and optic nerve head," Invest Ophthalmol Vis Sci, vol. 49, pp. 5103-10, 2008

[24] W. Wieser, B. R. Biedermann, T. Klein, C. M. Eigenwillig, and R. Huber, "Multi-Megahertz OCT: High quality 3D imaging at 20 million A-scans and 4.5 GVoxels per second," Optics Express, vol. 18 , pp. $14685-14704,2010$ 


\section{Ophthalmic OCT}

\section{Sponsors}

National Institutes of Health-RO1-EY11289-23, R01-EY13178-07, P30-EY008098

National Science Foundation-BES-0522845

Air Force Office of Scientific Research-FA9550-07-1-0101 and FA9550-07-1-0014

\section{Project Staff}

Prof. James G. Fujimoto, Dr. Bernhard Baumann, Dr. Ben Potsaid, WooJhon Choi, Martin Kraus, Jonathan J. Liu (MIT)

Laurel Vuong, Alan Sull, Joseph Ho, Dr. Jay S. Duker (New England Eye Center)

Dr. Joel Schuman (University of Pittsburgh Medical Center)

Dr. Allen Clermont, Dr. Edward Feener (Joslin Diabetes Center)

\subsection{OCT in Ophthalmology}

Optical coherence tomography (OCT) has had the largest clinical impact in ophthalmology. OCT is noncontact and can perform micron scale, cross sectional and 3D imaging of tissue morphology in real time. In ophthalmology, OCT provides information that is impossible to obtain by any other non-invasive technique. OCT has become a standard diagnostic tool in ophthalmology, enabling more sensitive diagnosis of disease, elucidating disease pathogenesis, progression and response to treatment in major retinal diseases such as age-related macular degeneration, diabetic retinopathy and glaucoma.

Our group and collaborators were responsible for the invention and initial development of OCT. The first report of OCT was published in Science in 1991 and demonstrated ex vivo imaging of the human retina and atherosclerotic plaque [1]. Our initial studies focused on ophthalmic imaging [2-4]. We developed a prototype clinical OCT system and in 1993 began clinical OCT imaging studies in collaboration with investigators Drs. Carmen Puliafito, M.D. and Joel Schuman, M.D. at the New England Eye Center (NEEC). Several thousand patients were imaged at the New England Eye Center using our prototype instrument during the 1990s. Our group performed many of the first cross-sectional and longitudinal studies of a wide range of ophthalmic diseases. For example, our group performed the first studies that suggested OCT measurements of nerve fiber layer (NFL) thickness will be useful for diagnosis and monitoring of glaucoma [5-7]. We also performed the first studies demonstrating OCT mapping of macular thickness in retinal edema [8]. We developed imaging protocols that became clinical standards used in commercial OCT instruments (StratusOCT from Carl Zeiss Meditec) [2-4] OCT technology was patented and our group in collaboration with Dr. Carmen Puliafito, M.D., (now Dean of the Keck School of Medicine at USC) and Eric Swanson (co-founder of Sycamore Networks) transferred the technology to Carl Zeiss Meditec. The first commercial clinical OCT instrument was introduced into the ophthalmic market in 1996. OCT gained widespread clinical acceptance with the introduction of the third generation instrument, the Stratus OCT (Carl Zeiss Meditec) in 2002 and is now a standard imaging method in ophthalmology.

Recent advances in OCT technology using spectral / Fourier domain detection techniques have dramatically increased imaging speeds and performance. These new developments achieved an increase of imaging speeds by a factor of $\sim 50-100$ and enabled the acquisition of $3 \mathrm{D}$ volumetric images within only a few seconds [9-18]. Early studies demonstrated the generation of en face OCT fundus images from raster scanned 3D-OCT data sets which enable precise registration of cross sectional images to fundus features $[16,19] .3 \mathrm{D}$ rendering and visualization of the normal retina and retinal pathologies $[18,20]$ and thickness mapping of retinal nerve fiber layer and intra-retinal layers has also been demonstrated [18]. In 2006, several companies introduced spectral / Fourier domain OCT instruments for ophthalmology. Today, commercial clinical OCT instruments based on spectral / Fourier domain OCT detection operate at $\sim 20,000-50,000$ axial scans per second, more than 50 times faster than previous generation instruments.

Previous OCT systems were based on time-domain detection, where a low coherence light source is used with a Michelson interferometer with a sample arm and a reference arm with a scanning delay. Measurements of the echo time delay of backscattered or backreflected light are performed by 
mechanically scanning the optical reference path (Figure 2.1A) [1]. Time-domain OCT has made a significant clinical impact in the field of ophthalmology and lead to the Stratus OCT (Carl Zeiss Meditec, Dublin, CA) system for retinal OCT imaging which in widespread clinical use.

Fourier domain OCT measures echo time delays of light by Fourier transforming the spectrum of the interferometer output signal. Different echo time delays of light produce different frequencies of fringes in the interference spectrum. This can also be understood by recognizing that a Michelson interferometer with a path delay acts like a frequency filter, whose periodicity depends on the path length mismatch. Fourier domain detection can be implemented in two ways: either by recording the entire interference spectrum simultaneously using a spectrometer with a multichannel analyzer (spectral / Fourier domain OCT, Fig. 2.1b) [11, 16, 17, 21, 22] or by using a rapidly tunable laser source and a photodiode or a pair of photodiodes (swept source / Fourier domain OCT, Fig. 2.1c) [23-26]. The retinal scanning module in the patient interface is the same for all three OCT detection technologies and shown in Fig. 2.1d.
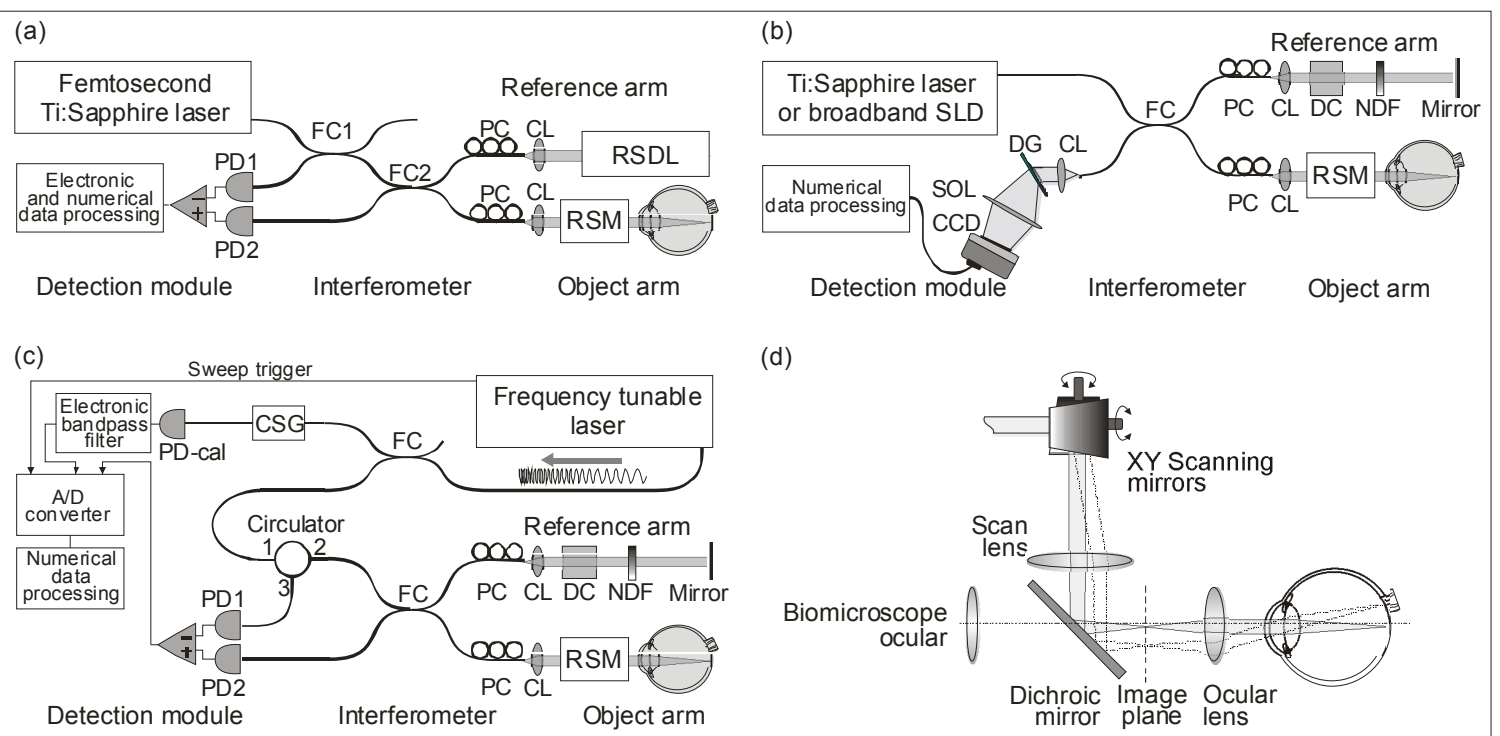

Figure 2.1. Schematic of OCT technologies used for ophthalmic imaging. (A) Time-domain OCT. (B) Spectral / Fourier domain OCT. (C) Swept source / Fourier domain OCT. (D) Retinal scanning module common to all three methods of OCT. Fourier domain detection methods (B-C) enable dramatic increases in sensitivity and speed, enabling three dimensional OCT imaging.

Our group developed ultrahigh resolution OCT for retinal imaging using broadband light sources and spectral / Fourier domain detection. In 2004, two years before the commercial introduction of spectral / Fourier domain OCT instruments, we began studies at the ophthalmology clinic of the New England Eye Center NEEC (Dr. Jay S. Duker, M.D., Chairman of Ophthalmology at the Tufts University School of Medicine) and the Eye Center of the University of Pittsburgh (Dr. Joel Schuman, M.D., Chairman of Ophthalmology at the University of Pittsburgh Medical School) using two prototype ultrahigh resolution spectral / Fourier domain instruments. Several thousand patients have been imaged to date and we have published many of the first studies using spectral / Fourier domain OCT in ophthalmology [18, 27-34].

In this report, we present an overview of our recent developments in ophthalmic OCT, with emphasis on technological advances which enhance speed and imaging performance. We present retinal imaging using ultrahigh speed spectral / Fourier domain OCT at $800 \mathrm{~nm}$ and $1050 \mathrm{~nm}$ wavelengths for imaging with ultrahigh resolution of $\sim 3 \mathrm{um}$ and enhanced penetration into the choroid and optic nerve (section 2.2). Recent results using swept source / Fourier domain OCT at $1050 \mathrm{~nm}$ wavelengths for retinal imaging with record speeds of 100,000 - 400,000 axial scans per second are presented in section 2.3. Ultrahigh speed Doppler OCT was developed and employed for 3D imaging of retinal blood flow (section 2.4). 
Another active area of research is structural and functional OCT imaging in the murine (rat and mouse) eye model (section 2.5). Finally, we give an overview of clinical studies conducted with our collaborators (section 2.6).

\subsection{Ultrahigh Speed Spectral / Fourier Domain OCT Retinal Imaging}

Early ophthalmic OCT instruments used time domain detection and because the image acquisition speeds were limited, it was only possible to acquire multiple cross-sectional images of the retina [1, 2, 35]. However, with the development of spectral / Fourier domain OCT [11, 21] and swept source / Fourier domain OCT [23,36], improvements in sensitivity and speed enabled the acquisition of three dimensional volumetric data sets using raster scanning. However, the current generation of commercial ophthalmic OCT instruments using spectral / Fourier domain detection operate at 25,000-29,000 axial scan per second imaging speeds $[37,38]$ which is still too slow to enable the acquisition of dense volumetric OCT data sets because the image acquistion times are limited by ocular motion and/or blinking. Higher imaging speeds are therefore important both to reduce the effects of eye motion as well as to obtain larger data sets with higher pixel resolutions.

Our group is investigating ultrahigh speed OCT for ophthalmic applications. Using spectral / Fourier domain detection, we have developed ultrahigh speed OCT research prototype instruments which can acquire high density volumetric data sets at speeds of $100,000-312,500$ axial scans per second at 850 $\mathrm{nm}$ using new CMOS line scan camera [39] and 47,000 axial scans per second at $1050 \mathrm{~nm}$ using InGaAs line scan cameras. These ultrahigh imaging speeds reduce eye motion artifacts and enable high transverse sampling density. Fig. 2.2a, shows an example of 3D OCT data of the macula obtained using a prototype $850 \mathrm{~nm}$ instrument operating at 250,000 axial scans per second. The resulting data sets represent the retinal morphology over large areas of the retina, facilitating precise measurement of retinal structures and correlation of data from visit to visit. Cross-sectional images, the current diagnostic standard, can be extracted from the 3D volume at arbitrary position and orientation for improved visualization of retinal features and pathologies. The dense transverse sampling within the volume also enables the generation of en face, OCT fundus images by axially summing the axial scans over the layer depth of interest at each transverse pixel in the image. These en face OCT fundus images can be used to visualize nerve fibers, retinal vessels, capillary networks and choroid vasculature, which cannot be easily visualized with cross-sectional images alone. As shown in Fig. 2.2b, densely sampled volumetric data acquired from the optic nerve head allows en face visualization of the nerve bundles within the lamina cribrosa, which may be an important marker for glaucoma.

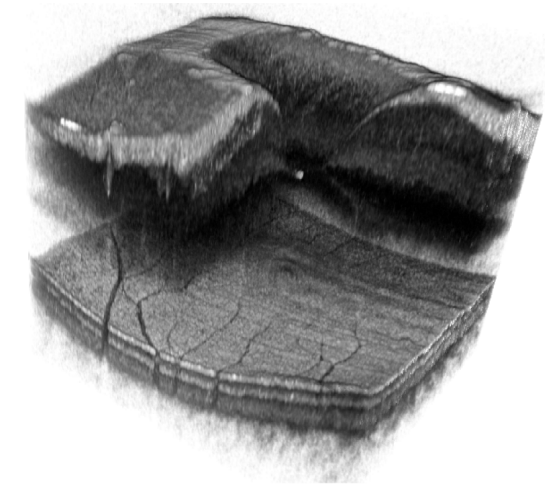

(a)

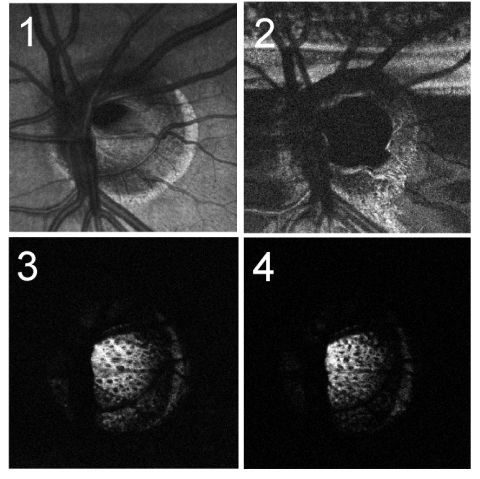

(b)

Figure 2.2. (a) $3 \mathrm{D}$ volumetric rendering of macula data consisting of $512 \times 512$ transverse pixels acquired at 250,000 axial scans per second using a spectral / Fourier domain OCT system at 850 $\mathrm{nm}$. (b) En face images generated from a 3D data set of the optic nerve head obtained at 106,000 axial scans per second at $850 \mathrm{~nm}$ showing (b1) OCT fundus view and en face visualization at different depths (b2)-(b4), with the lamina cribrosa in the optic nerve head visible at increase depths (b3)-(b4). 
Ultrahigh speed also facilitates the visualization of small features by reducing image distortion and blurring due to drift eye motion and micro-saccades. Fig. 2.3a shows a small volume of 3D data covering a $\sim 200$ um by $\sim 250$ um area of the retina acquired at 250,000 axial scans per second. Retinal capillaries located around the INL can be seen within the volume as shown in Fig. 2.3b, and the capillary network can be seen in an en face image generated by summing axial scans at the depth of the INL. Such 3D and en face OCT visualization techniques may enable new and more sensitive methods for diagnosis and assessing disease progression. Improved structural visualization promises to reduce the time span over which retinal changes and/or response to therapy can be detected and quantified.

Imaging at longer $1050 \mathrm{~nm}$ wavelengths improves image depth penetration because scattering is reduced compared with $850 \mathrm{~nm}$. Long wavelengths increase image penetration into the choroid and optic nerve head, as well as reduce signal attenunation in patients with occular opacities and cataracts [40, 41]. Figs. 2.3d-h show images obtained with our prototype $1050 \mathrm{~nm}$ spectral / Fourier domain system operating at 47,000 axial scans per second. A 3D rendering of the optic nerve head shows deep penetration into the choroid and optic nerve head, as shown in Fig. 2.3d. The OCT fundus view shows minimal motion artifacts in the transvers direction at these imaging speeds. The high transverse sample density in the volumes allows averaging of adjacent B-scans to reduce speckle and improve image contrast, as shown in Figs. 2.3f-g. OCT cross-sectional images with improved contrast and signal to noise can be generated at arbitrary positions and orientations by interpolating and averaging the volumetric data set. Fig. 2.3h. compares images generated by averaging adjacent B-scans within a volume to images generated by averaging cross-sectional images acquired by repeated scanning the same retinal position.

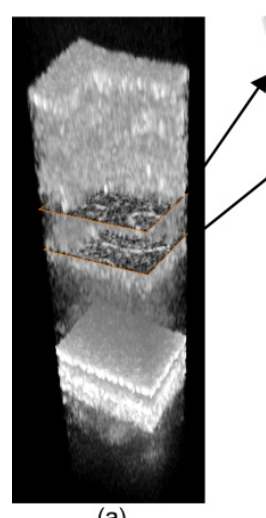

(a)

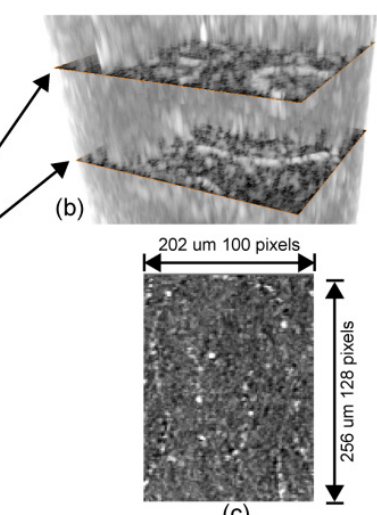

(c)
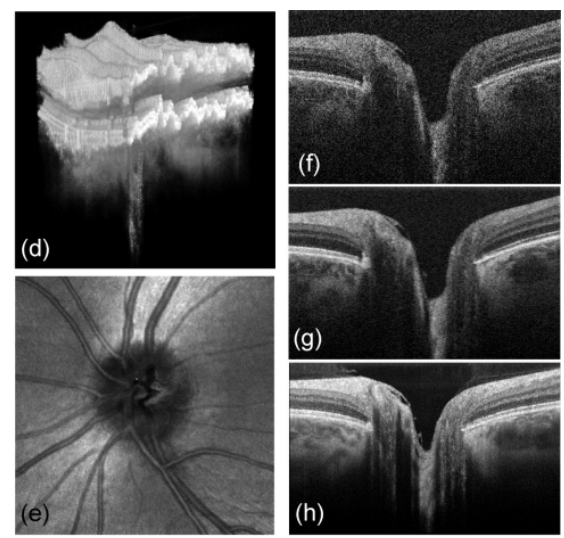

Figure 2.3. (a) 3D volumetric rendering of retinal layers acquired at 250,000 axial scans per second $(850 \mathrm{~nm})$. (b) Retinal capillaries shown above and below the INL. (c) En face visualization of the retinal capillaries showing blood flow by subtracting successive images from a repeated volume sequence. (d) 3D volumetric rendering of optic nerve head consisting of $400 \times 400$ transverse pixels acquired at 47,000 axial scans per second $(1050 \mathrm{~nm})$. (e) OCT fundus view of data shown in (d). (f) single cross sectional image extracted from (d). (g) Average of 4 adjacent Bscans from the volume shown in (d). (h) Average of 8 images acquired with a rapidly repeated single line scan protocol consisting of 2000 pixels acquired at 47,000 axial scans per second (1050 $\mathrm{nm})$.

Two prototype spectral / Fourier domain OCT imaging systems were developed for clinical use and deployed at the Tufts University / Tufts Medical New England Eye Center. Figure 2.4 shows images obtained with an $850 \mathrm{~nm}$ and a $1050 \mathrm{~nm}$ OCT system. The instrument operating at $850 \mathrm{~nm}$ can achieve ultrahigh axial image resolutions of $\sim 3 \mathrm{um}$. The figure shows images from patient $A$ (right eye of a 67 year old subject diagnosed with multifocal choroiditis), which are extracted from 3D data set of a 400x400 axial scan volume acquired in only 2.2 seconds. The instrument operating at $1050 \mathrm{~nm}$ achieved deep image penetration, but has a standard $\sim 7$ um axial resolution. The figure shows images from patient $B$ (Left eye of a 61 year old subject with wet age related macular degeneration), which are extracted from a 300x300 axial scan volume acquired in 2.3 seconds. 

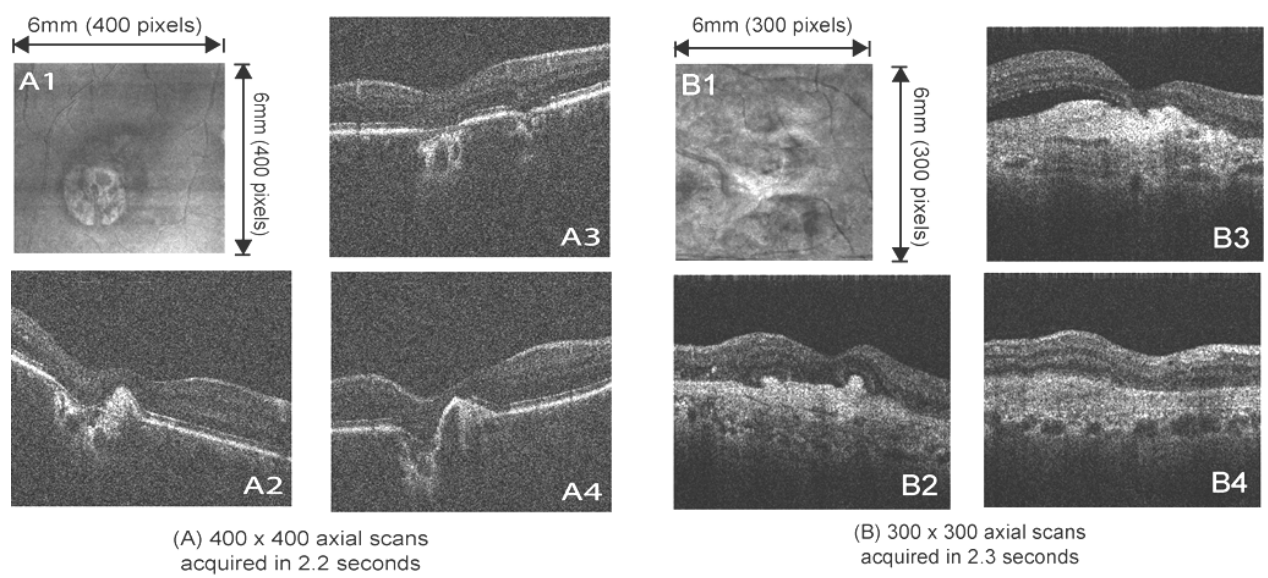

Figure 2.4. (A) Pathology from patient $A$ imaged with the $91 \mathrm{kHz}$ axial scan rate $850 \mathrm{~nm}$ wavelength CMOS linescan camera based spectral / Fourier domain instrument. (A1) OCT fundus image of patient $A$ diagnosed with multifocal choroiditis. (A2) $Y-Z$ and (A3)-(A4) X-Z cross sectional images of patient $A$ extracted from a $400 \times 400$ axial scan volume. (B) Pathology from patient $B$ imaged with the $47 \mathrm{kHz}$ axial scan rate $1050 \mathrm{~nm}$ wavelength InGaAs linescan camera based spectral / Fourier domain instrument. (B1) OCT fundus image of patient $B$ diagnosed with wet age-related macular degeneration. (B2) $\mathrm{Y}-\mathrm{Z}$ and (B3)-(B4) $\mathrm{X}-\mathrm{Z}$ cross sectional images of patient $\mathrm{B}$ extracted from a $300 \times 300$ axial scan volume.

The results of these studies suggest that ultrahigh speed OCT and dense volumetric data sets can achieve a significant improvement in performance for ophthalmic imaging. Volumetric data promises to have a powerful impact in clinical applications, improving early diagnosis, reproducibility of quantitative measurements and enabling more sensitive assessment of disease progression or response to therapy.

\subsection{Swept Source / Fourier domain OCT Imaging at $1050 \mathrm{~nm}$ Wavelengths}

Current commercial ophthalmic OCT retinal imaging systems use spectral/Fourier domain detection with spectrometers with CCD cameras operating at imaging speeds of $\sim 25-52 \mathrm{kHz}$ axial scan rate. Broadband SLD light sources at $800 \mathrm{~nm}$ are used to achieve $\sim 5-7 \mathrm{um}$ axial resolution in tissue. Swept source/Fourier domain OCT uses a wavelength swept laser source with photodiodes and a high speed A/D converter [23, 36]. Swept source/Fourier domain OCT has advantages over spectral/Fourier domain OCT including: reduced fringe washout, longer imaging range (reduced sensitivity roll off with depth), higher detection efficiencies because there are no spectrometer losses and the ability to perform dual balanced detection to reduce noise.

Our group is developing ultrahigh speed, swept source/Fourier domain OCT systems for ophthalmic imaging of the retina and anterior segment using short cavity swept laser technology. We are also working in collaboration with Thorlabs and Praevium Research to develop and validate the next generation of swept laser technology which will improve imaging speeds and imaging range. The short round trip times of short cavities enable a significant increase in sweep speeds over conventional swept laser technology because the sweep speed is governed by the time needed to build up lasing from spontaneous emission noise to saturate the gain medium as the laser is dynamically tuned [4].

Using a data acquisition clock signal derived from the optical sweep signal, the A/D converter can be clocked at variable rate, such that the OCT fringe is automatically sampled to be linear in $\mathrm{k}$ (wavenumber) or frequency, even with nonlinear sweeps and/or sweep-to-sweep variation [5,6]. Figure 2.5A shows an interferometric OCT fringe from a shallow and deep mirror reflection, with phase indicated by the curve in red. When the same fringe is sampled using the optical clocking scheme, the phase is linearized at acquisition. This has the important advantage that numerical processing to re-sample the fringe signal is not required and data processing speeds can be significantly enhanced. Figure 2.5B-D show the 
sensitivity roll-off performance of the OCT systems using optical clocking, fixed 400 MSPS and fixed 1 GSPS sampling. The maximum imaging ranges are long and the long coherence length of the short cavity laser results in superior sensitivity roll-off when compare to spectral/Fourier domain and conventional swept source OCT technology.
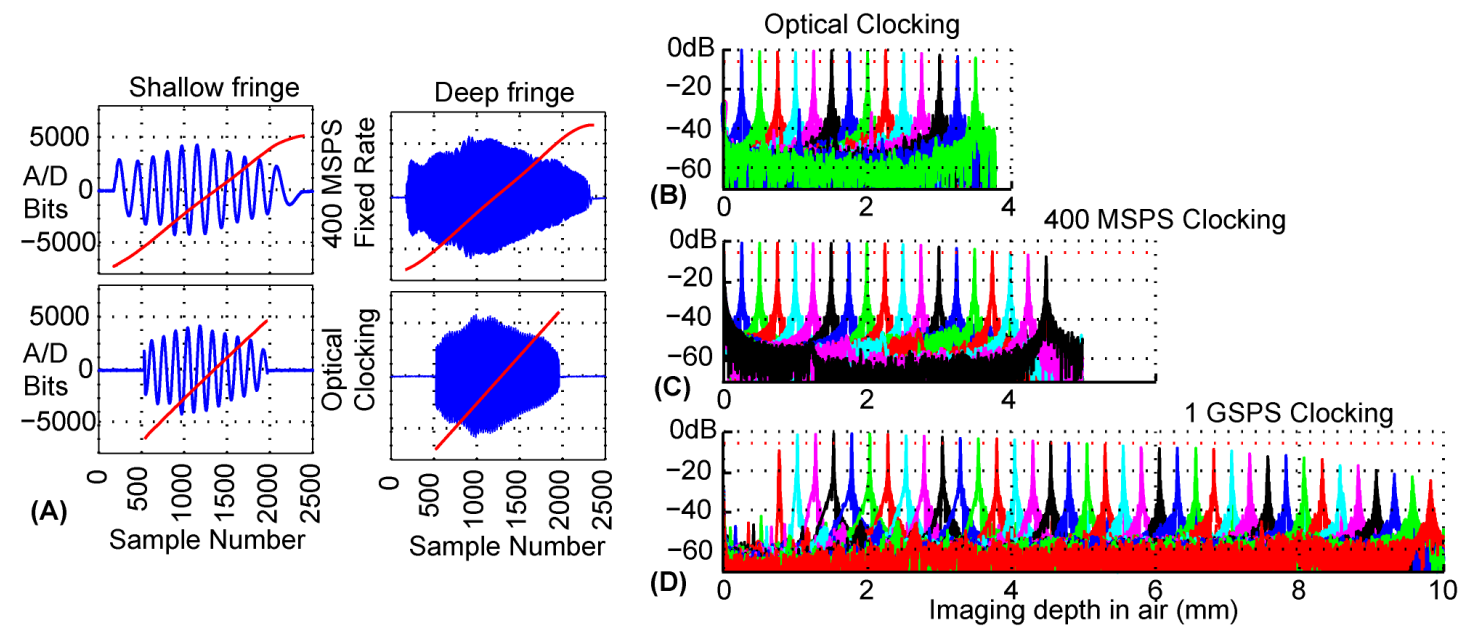

Figure 2.5. (A) OCT fringe data from a mirror at a shallow depth (left) and deep position (right) acquired with a fixed 400 MSPS rate (top) or optical clocking (bottom). Sensitivity roll-off performance for (A) optical clocking, (B) fixed 400 MSPS rate, and (C) fixed 1 GSPS rate acquisition.

Imaging speeds of $100 \mathrm{kHz}$ to $200 \mathrm{kHz}$ axial scan rate enable the acquisition of densely sampled 3D OCT data sets with minimal motion artifacts. Fig. 2.6A shows a 500x500 axial scan data set of the optic nerve head acquired at $100 \mathrm{kHz}$. Cross sectional OCT images of the macula (Fig. 2.6B) and disc (Fig. 2.6C) show deep penetration into the choroid and optic nerve head, enabled by the reduced optical scattering in tissues at $1050 \mathrm{~nm}$ wavelengths. The $5.3 \mathrm{um}$ axial resolution enables delineation of fine retinal layers. At $200 \mathrm{kHz}$ axial scan rate, large $12 \times 12 \mathrm{~mm} 3 \mathrm{D}$ data sets including both the macula and disc can be acquired (Figs. 2.6D, 2.6E, and 2.6F). This data set contains $30 \mathrm{X}$ more axial scans than a standard $200 \times 200$ volumetric cube acquired by commercial instruments and shows both the macula and optic disc in the same image.

Small area zoom imaging at $200 \mathrm{kHz}$ shows individual cone photoreceptors in certain regions of the retina (Fig. 2.7A-B). The high speeds enable visualization of small features by reducing image distortion and blurring due to motion. Imaging of the anterior eye can also be performed. Fig. 2.7C shows a cross sectional OCT image of the anterior angle with deep image penetration achieved at $1050 \mathrm{~nm}$ wavelengths. Outflow features, such as the tribecular meshwork and Schlemm's canal can be visualized. Figure 2D shows an extended imaging range configuration of the instrument with $7.5 \mathrm{~mm}$ depth range in tissue (10 $\mathrm{mm}$ in air) achieved by using a 1 MSPS sampling rate for the data acquisition. The cornea, iris, and front of the lens can be visualized in a single image using this long imaging range configuration.

3D volumetric data acquisition rates can be increased further by imaging using two beams, two interferometers and two balanced detectors in parallel. Figure 2.8 shows results obtained at 400,000 axial scan rate, which we believe is the fastest acquisition rate reported for in vivo for the human retina at this time. A large $12 \mathrm{~mm} \times 10 \mathrm{~mm}$ volume consisting of $1100 \times 930$ transverse pixels was acquired in 2.9 seconds, enabling comprehensive visualization of the optic disc and fovea within the same acquisition and within a reasonable acquisition time. 

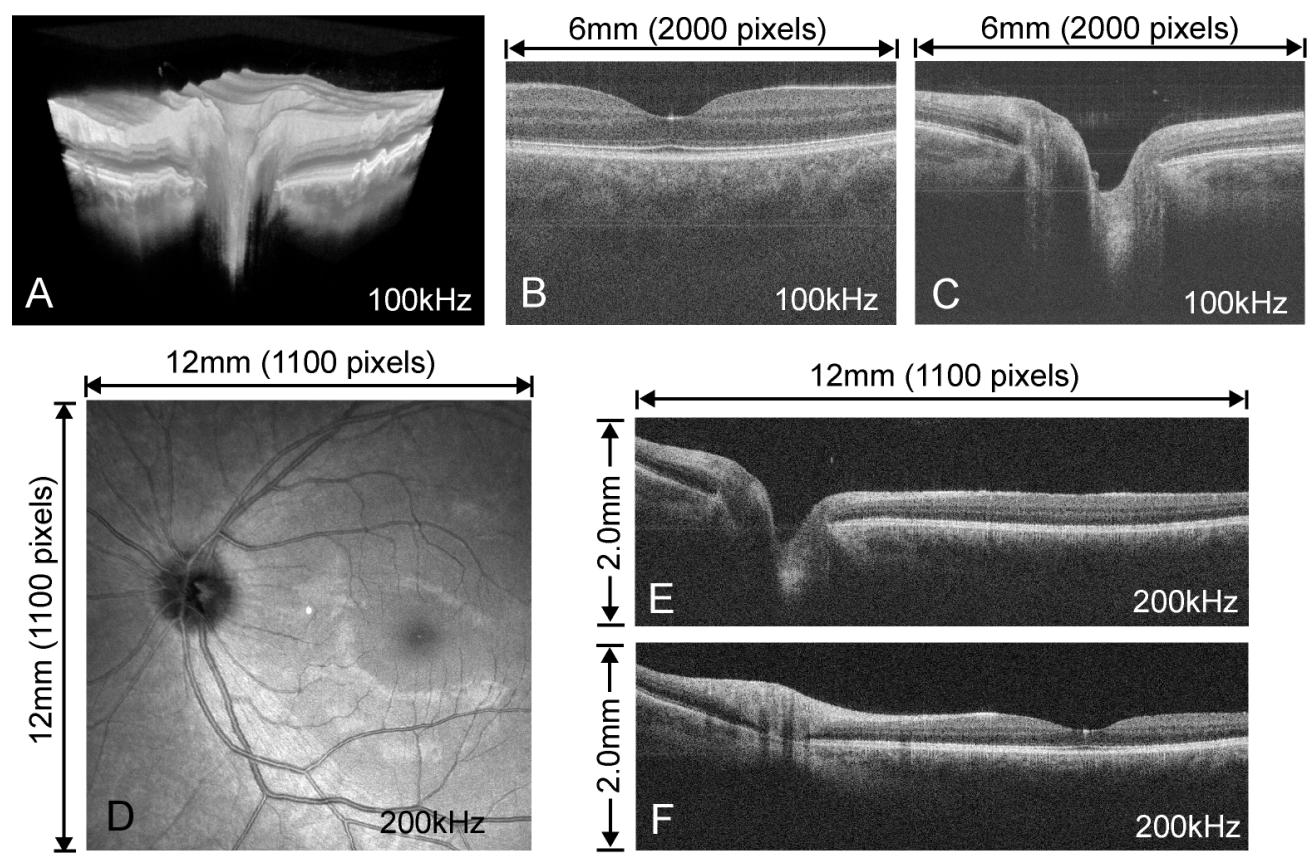

Figure 2.6. (A) 3D volumetric OCT data set of optic nerve head consisting of 500x500 transverse pixels acquired at $100 \mathrm{kHz}$ axial scan rate in 2.6 seconds. (B) Macula and (C) optic nerve head cross sectional OCT images acquired at $100 \mathrm{kHz}$ consisting of 2000 axial scans over $6 \mathrm{~mm}$. (D) OCT fundus view of large $12 \times 12 \mathrm{~mm} 3 \mathrm{D}$ data set acquired at $200 \mathrm{kHz}$ consisting of $1100 \times 1100$ axial scans. (E) and (F) cross sectional OCT images extracted from the volume shown in (D).
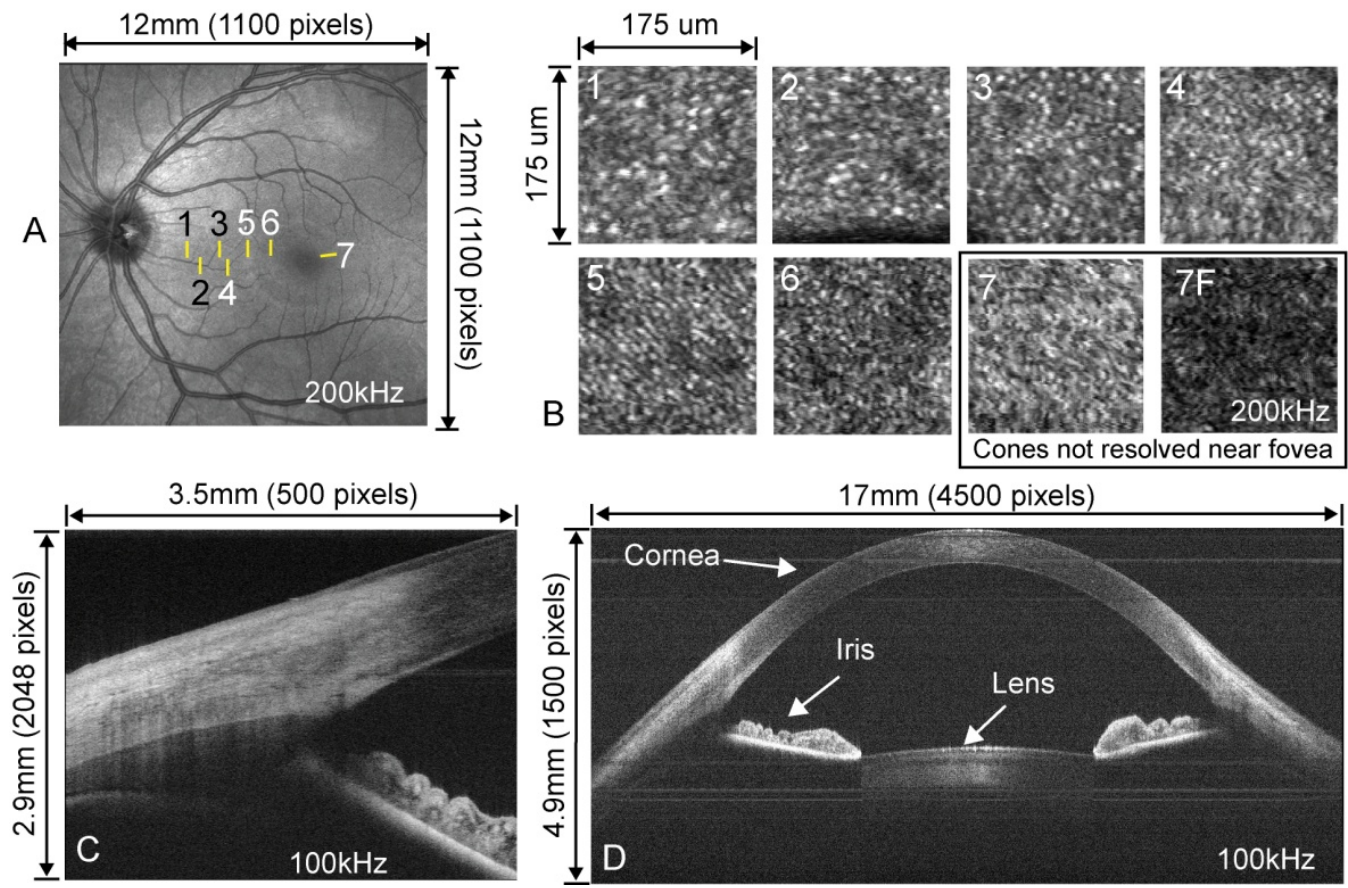

Figure 2.7. (A) $12 \mathrm{~mm} \times 12 \mathrm{~mm}$ OCT fundus image showing regions 1-7 where zoom imaging was performed. (B) En face images from regions 1-7 obtained by summing linearly scaled intensity data over the photoreceptor depth range. Decreasing cone size and spacing can be observed moving from the disc to fovea. (C) OCT cross sectional image of the anterior angle (average of 2 images). (D) A long imaging range system configuration with $7.5 \mathrm{~mm}$ range enables viewing the anterior segment, including the cornea, iris and front of the lens. 


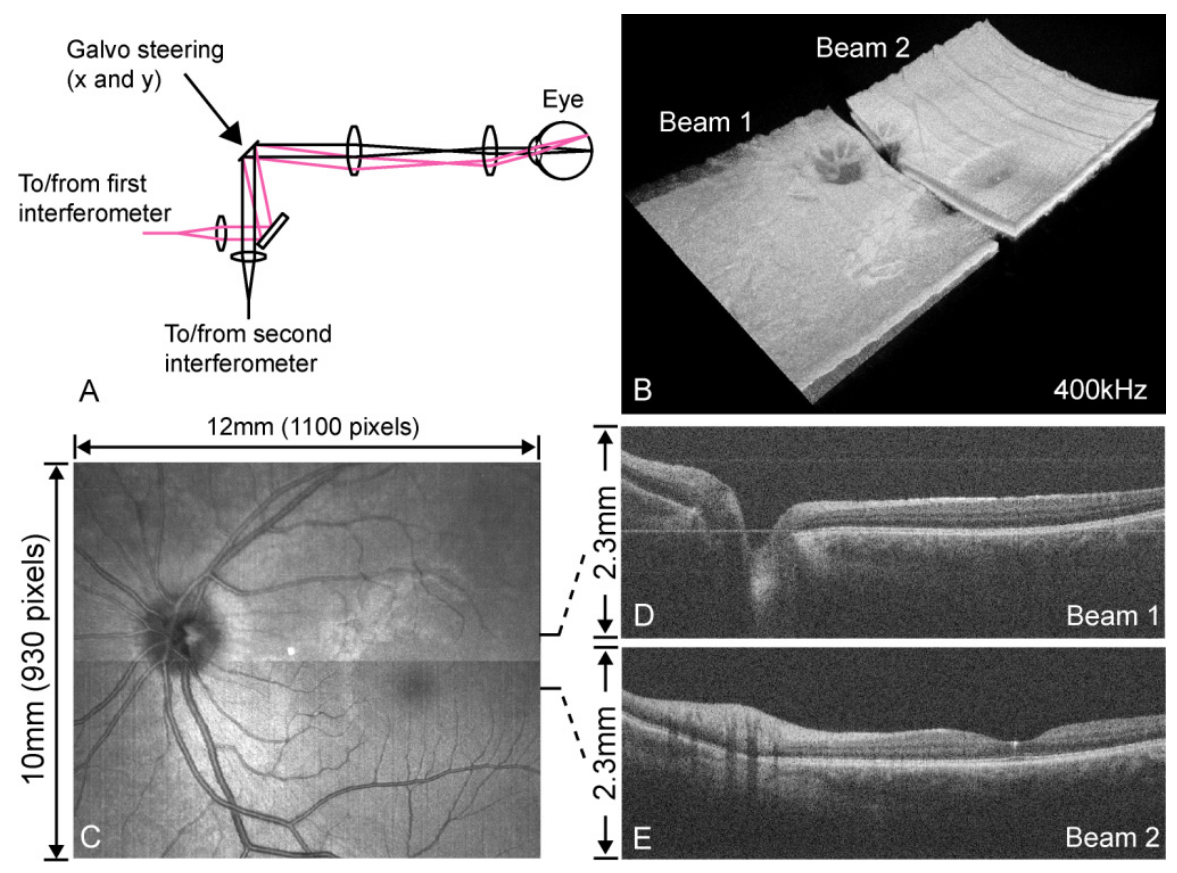

Figure 2.8. (A) Dual beam OCT imaging at $400 \mathrm{kHz}$ axial scan rate. (A) Dual beam patient interface. (B) $3 \mathrm{D}$ rendering showing the two independent volumes acquired in parallel. (C) OCT fundus image of registered 3D date. (D) Cross sectional OCT image from the first beam. (E) Cross sectional OCT image from the second beam.

The improvements in imaging speed and depth range with swept source / Fourier domain OCT and short cavity lasers provide important advantages for ophthalmic imaging. The ability to rapidly acquire 3D-OCT data over a wide field of view promises to simplify examination protocols. The ability to image fine structures can provide detailed information on focal pathologies. The large imaging range and improved image penetration at $1050 \mathrm{~nm}$ wavelengths promises to improve performance for instrumentation which images both the retina and anterior eye. These advantages suggest that swept source OCT at $1050 \mathrm{~nm}$ wavelengths will play an important role in future ophthalmic instrumentation. Additionally, sweep buffering and multibeam OCT acquisition promise to be important enabling technologies for ultrahigh speed OCT imaging in ophthalmology and other applications.

\subsection{Doppler OCT Imaging}

Doppler optical coherence tomography (OCT) or optical Doppler tomography is a functional extension of OCT [42-45]. In addition to OCT's ability to perform high-speed, three-dimensional (3D) imaging of transparent or translucent structures with micrometer resolution, Doppler OCT enables volumetric imaging of retinal blood flow [46, 47]. Abnormalities in retinal blood flow are related to several ocular diseases such as glaucoma, diabetic retinopathy and age-related macular degeneration. Accurate knowledge of ocular perfusion is important not only for treatment, but also for understanding the pathophysiology of these diseases, which are the most common causes of blindness in the industrialized world. Hence, there is great demand for methods to assess of ocular blood flow in vivo.

Similar to color Doppler imaging using ultrasound, Doppler OCT relies on the Doppler effect [42]. Moving scatterers induce a phase shift in the OCT signal. The magnitude of this Doppler shift is proportional to the flow velocity component in direction of the OCT beam. Two- and three-dimensional images of retinal blood flow can be generated by evaluating these Doppler shifts in the OCT data [42-47]. Doppler OCT methods for assessment of absolute flow velocities and volumetric flow values usually require knowledge of vascular geometry, modification of the OCT system's optics or specialized scan patterns, since only the flow velocity component in probe beam direction can be measured [48-50] . 
In this study, a novel approach is presented to assess absolute flow velocities as well as total retinal blood flow in vivo. Unlike previous methods, knowledge of the Doppler angle, i.e. the angle between vessel and probe beam, is not required. Absolute flow values can be computed from 3D Doppler OCT data by integrating the flow components in a plane bisecting vessels perpendicular to the probe beam, i.e., in an en-face plane [51]. In most parts of the retina, the blood vessels are almost parallel to the retinal surface. If blood flow is to be determined using this approach, the central retinal vessels in the optic disk must be imaged. In the optic disk, the vessels are almost parallel to the OCT beam, and all vessels can be covered by a volumetric data cube. However, the flow velocity components in OCT beam direction are high (up to $\sim 50 \mathrm{~mm} / \mathrm{s}$ ), therefore high axial scan rate and deep penetration into the optic nerve is required.

An ultrahigh-speed, swept-source OCT system at $1050 \mathrm{~nm}$ was developed which has multiple advantages for quantitative imaging of rapid retinal blood flow [52]. Imaging at $1050 \mathrm{~nm}$ wavelengths provides enhanced penetration into deep ocular structures [40,53,54]. Second, high axial scan rates enable detection of high flow velocities even in the central retinal vessels. Third, swept-source OCT is less sensitive to phase washout compared to spectrometer-based Fourier domain systems [55, 56]. In our prototype system, the sweep rate of the laser light source was $\sim 100 \mathrm{kHz}$. The laser emitted light only during the forward sweep, which results in a duty cycle of $\sim 50 \%$. The laser sweep rate was doubled to $\sim 200 \mathrm{kHz}$ by buffering with a fiber delay of $\sim 5$ us. With an A/D sampling rate of $400 \mathrm{MHz}$, the image range was $3.8 \mathrm{~mm}$ in tissue. The power of the OCT light beam at the cornea was $1.8 \mathrm{~mW}$ and the sensitivity of the system was $95 \mathrm{~dB}$.

Figure 2.9 shows results of retinal blood flow measurements using the ultrahigh-speed OCT system. 3D data sets of $250 \times 2000$ axial scans covering an area of $3 \mathrm{~mm} \times 3 \mathrm{~mm}$ around the optic disk were recorded in the eyes of healthy volunteers. Doppler images were computed between every other (100 $\mathrm{kHz}$ ) and every axial scan (200 kHz). 3D renderings are shown in Figs. 2.9A and $\mathbf{B}$ for $100 \mathrm{kHz}$ and 200 $\mathrm{kHz}$, respectively. The treelike structure of the central retinal vessels branches can be appreciated nicely in these images. Even in the central retinal arteries deep in the papilla, blood flow can be observed when imaging at $200 \mathrm{kHz}$. Figs. 2.9B and $\mathbf{D}$ show examples of Doppler B-scan images at $100 \mathrm{kHz}$ and $200 \mathrm{kHz}$. Note the different velocity ranges for the two images. At $100 \mathrm{kHz}$ axial scan rates, there is still phase wrapping in the central retinal vessels where the flow velocities are high. However, no such artifacts are observed at $200 \mathrm{kHz}$. Total flow was measured in the optic disk by integrating over the vessels in an en face projection. Flow values were $11.6 \mathrm{uL} / \mathrm{min}, 24.4 \mathrm{uL} / \mathrm{min}$, and $47.5 \mathrm{uL} / \mathrm{min}$ in the retinal arteries $\mathrm{A} 1, \mathrm{~A} 2$, and $A 3$ in the en-face image of Fig. 2.9E.
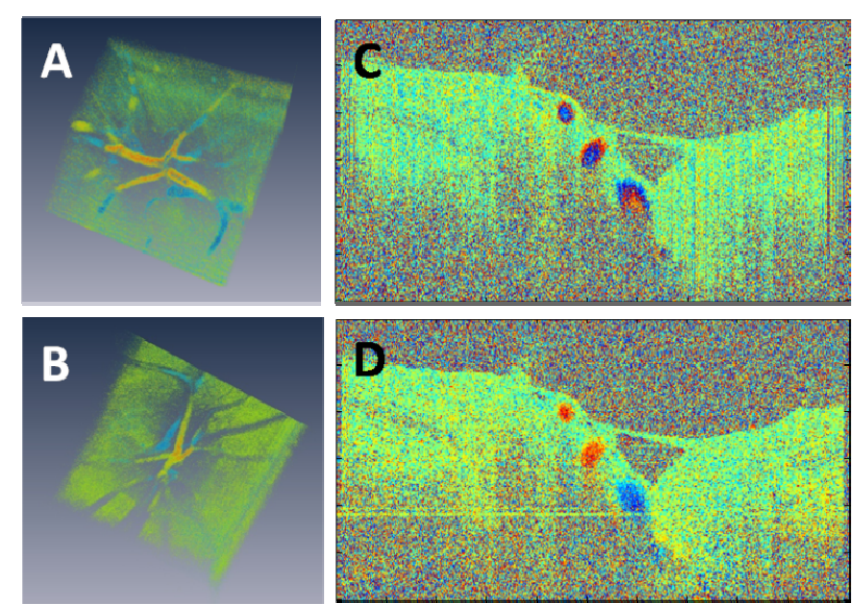

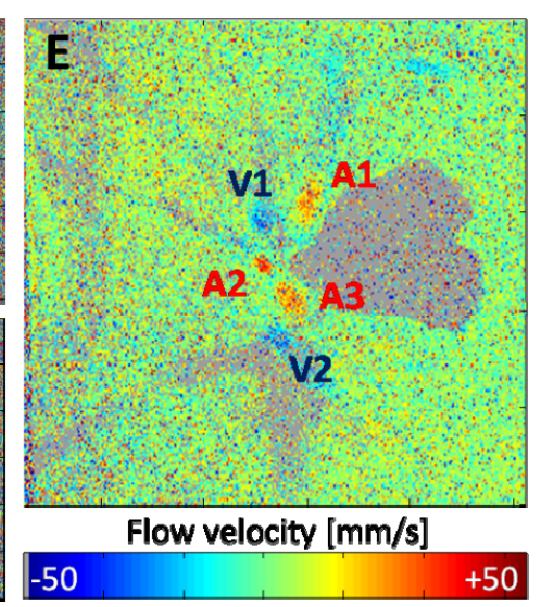

Figure 2.9. Ultrahigh-speed Doppler OCT images of the optic disk. (A) Volume rendering at 100 $\mathrm{kHz}$ showing the vasculature. (B) Volume rendering at $200 \mathrm{kHz}$ allows for visualization of the central retinal arteries' branching. (C) Doppler B-scan image at $100 \mathrm{kHz}$. (D) Doppler B-scan image at $200 \mathrm{kHz}$. (E) En-face image showing cross sections of central retinal arteries $(A)$ and veins $(V)$. The color scale in $(A)$ and $(C)$ ranges from -25 to $+25 \mathrm{~mm} / \mathrm{s}$. 
These results demonstrate the ability of ultrahigh-speed swept-source OCT for retinal blood flow imaging. Swept source OCT promises to be a powerful tool for in vivo assessment of blood flow in healthy and diseased eyes.

\subsection{Small Animal Retinal Imaging}

Because it can perform non-invasive, three dimensional imaging of retinal structures with ultrahigh resolution, optical coherence tomography is well suited for ophthalmic imaging in animals. Animal models are valuable for ocular research due to the inherent limitations on studying pathophysiologic processes in the human eye. Since the Murine (rat and mouse) eye has similaritie to the human eye and there are multiple transgenic mouse models of retinal disease, murine models of ocular disease provide powerful tools for the analysis and characterization of disease pathogenesis and response to treatment. While enucleation and histology provide the gold standard to characterize microstructural changes in animals, non-invasive structural imaging can be performed repeatedly to track disease progression and has the potential to reduce the need for sacrifice and histology in many studies [57].

We developed an ultrahigh resolution spectral / Fourier domain OCT prototype instrument for murine retinal imaging using new, high-speed CMOS imaging technology. This instrument can achieve imaging speeds $>70,000$ axial scans per second and axial image resolutions of $\sim 3$ um [39]. A microscope delivery system is used to focus and scan the OCT beam in the animal eye. In addition to 3D-OCT data sets of the rat retina, Doppler OCT analysis of blood flow in the rat retina can be performed. Doppler OCT provides non-invasive in vivo quantitative measurements of retinal blood flow properties [44, 46, 58], and it may benefit studies involving glaucoma and diabetic retinopathy. Ultrahigh-resolution spectral/Fourier-domain OCT promises to enable novel protocols to measure retinal structure and retinal blood flow in small animals.

Working in collaboration with Dr. Edward Feener and Alan Clermont from the Joslin Diabetes Center, we developed and apply new technology for high-speed, ultrahigh resolution optical coherence tomography (OCT) technology to image and quantitatively assess retinal edema in animal models as the result of vascular permeability changes. In addition to hardware development, we have developed software to generate retinal thickness maps from data acquired with commercial OCT devices for small animal imaging, such as the Bioptigen SDOCT imaging system.
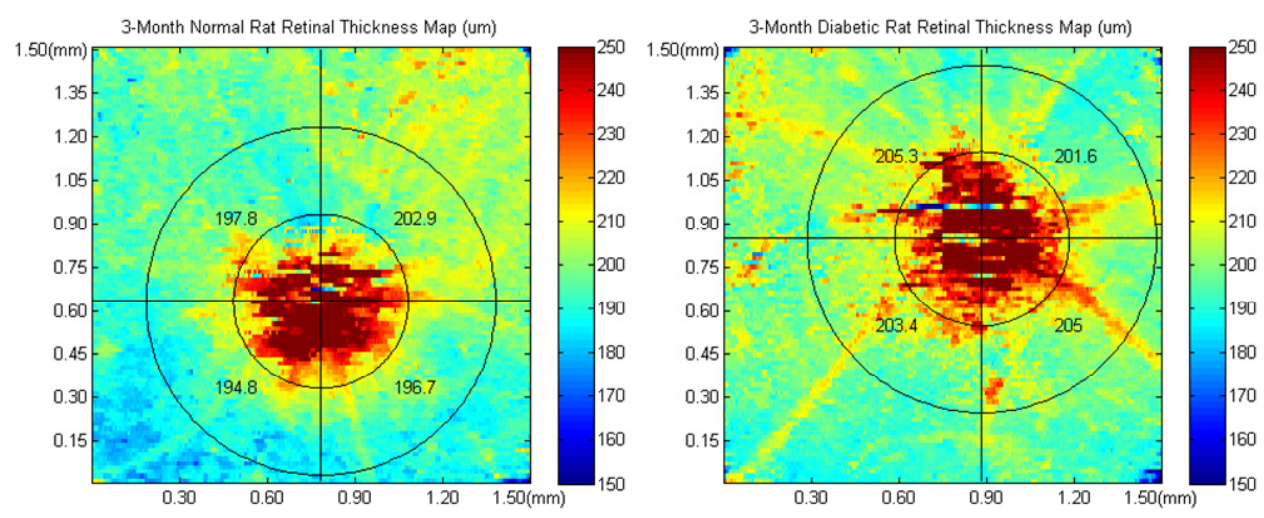

Figure 2.10. Examples of topgraphic retinal thickness maps obtained from 3D volumetric data. Thickness map from normal rat (left) compared with diabetic rat (right) which exhibits increased retinal thickness (edema) from changes in vascular permeability.

\subsection{Clinical OCT Studies}

Ultrahigh resolution spectral / Fourier domain OCT provides significantly increased sensitivities, imaging speed, and image quality. The high speeds enable raster scanning, which improved retinal coverage, 
thereby avoiding sampling errors in the detection of focal pathologies, and enabled three-dimensional (3D) volumetric imaging while preserving retinal topography [27]. 3D-OCT datasets provide comprehensive information about retinal structure and can be used to extract retinal layer thickness maps, retinal nerve fiber layer thickness maps, and optic nerve head contour information. OCT fundus images that show features such as blood vessels can also be created by using 3D-OCT data. Individual cross-sectional OCT images are automatically registered to the OCT fundus image. This enables correlation of 3D-OCT findings with standard ophthalmic examination.

Clinical studies using several generations of prototype OCT instruments have been performed at the New England Eye Center, the University of Pittsburgh Medical Center, and collaborating institutions in the past 15 years. Our high-speed ultrahigh resolution OCT prototype provides new information about retinal structure that complements standard diagnostic techniques such as fluorescein angiography (FA), indocyanine green angiography (ICG) or fundus photography by providing cross-sectional images of micron-scale pathological changes. In order to take full advantage of this ability in ophthalmology, systematic cross-sectional and longitudinal studies of pathologies are necessary. These studies illustrate features that are characteristic of various retinal pathologies and aid ophthalmologists in the interpretation of OCT images.

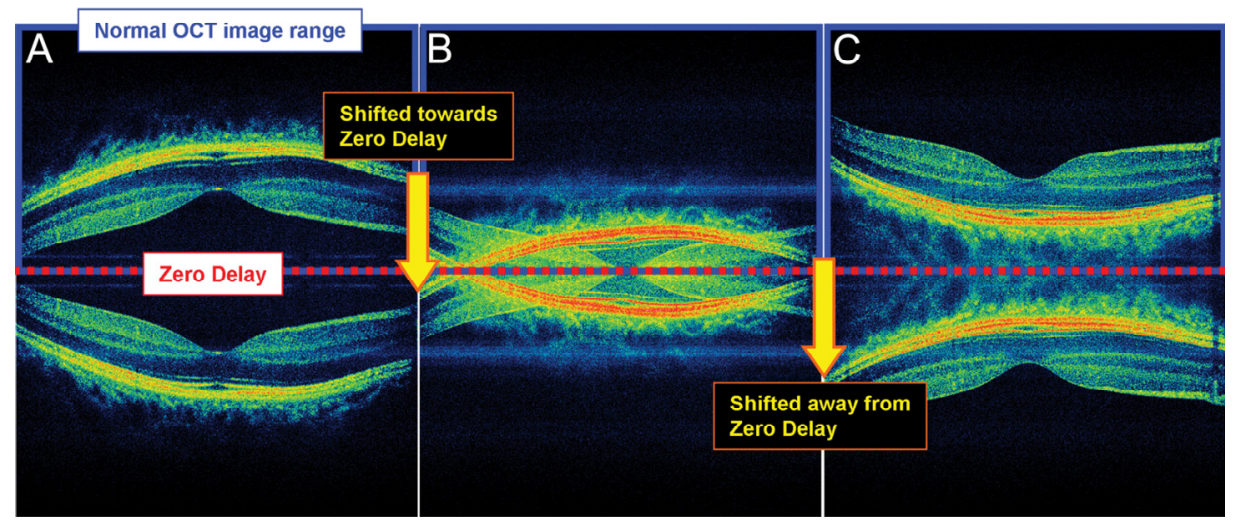

Figure 2.11. Mirror artifacts in SD/FD-OCT imaging. (A) SD/FD-OCT images are symmetrical about the zero-delay line after Fourier transformation. Half of the image is usually removed during image processing. The inner retina of both portions of the symmetric image is closer to the zero-delay line than is the choroid. (B) When part of the visible image crosses the zero-delay line, the other half of the symmetric image (usually cropped out) simultaneously crosses into the visible half of the scan box. This is when the mirror artifact is most apparent. (C) When the OCT image continues to be moved towards the zero-delay line, the choroid in both halves of the symmetric image is closer to the zero-delay line than is the inner retina.

Commercial SD/FD-OCT instruments were introduced to the market in 2006. Presently, there are more than 7 companies in the ophthalmic market. Standard commercial instruments now achieve speeds of 25,000 axial scans per second with 5 to 7 um axial image resolution. We have reported normal macular thickness measurements and assessed reproducibility of retinal thickness measurements acquired by a time-domain OCT (Stratus, Carl Zeiss Meditec, Inc., Dublin, CA) and three commercially available pectral/Fourier domain OCT instruments (Cirrus HD-OCT, Carl Zeiss Meditec, Inc.; RTVue-100, Optovue, Inc., Fremont, CA; 3D OCT-1000, Topcon, Inc., Paramus, NJ) [59]. We have also reported the frequency of OCT scan artifacts and compared macular thickness measurements, interscan reproducibility, and interdevice agreeability across the 3 SD-OCT systems [60]. We further investigated the characteristics of a spectral-domain optical coherence tomography (SD-OCT) image phenomenon known as the mirror artifact and found that this image artifact was often associated with patients with moderate to high myopia [61]. In a separate study, we examined techniques to make TD-OCT circular scan RNFL thickness measurements comparable with those from 3D SD-OCT volumes. We found that scan location matching is important in RNFL thickness measurements with TD-OCT circular scan data and 3D SD-OCT scan data to provide follow-up comparability across the two generations of OCTs [62]. In another study, we 
examined choroidal thickness and area in healthy eyes using SD-OCT. Choroidal thickness across the macula demonstrates a thin choroid nasally, thickest subfoveally, and again thinner temporally, and a trend toward decreasing choroidal thickness with age [63].

\section{References}

[1] D. Huang, E. A. Swanson, C. P. Lin, J. S. Schuman, W. G. Stinson, W. Chang, M. R. Hee, T. Flotte, K. Gregory, C. A. Puliafito, and J. G. Fujimoto, "Optical Coherence Tomography," Science, vol. 254, pp. 1178-1181, 1991

[2] E. A. Swanson, J. A. Izatt, M. R. Hee, D. Huang, C. P. Lin, J. S. Schuman, C. A. Puliafito, and J. G. Fujimoto, "In vivo retinal imaging by optical coherence tomography," Optics Letters, vol. 18, pp. 1864-1866, 1993

[3] M. R. Hee, J. A. Izatt, E. A. Swanson, D. Huang, J. S. Schuman, C. P. Lin, C. A. Puliafito, and J. G. Fujimoto, "Optical coherence tomography of the human retina," Archives of Ophthalmology, vol. 113, pp. 325-332, 1995

[4] C. A. Puliafito, M. R. Hee, C. P. Lin, E. Reichel, J. S. Schuman, J. S. Duker, J. A. Izatt, E. A. Swanson, and J. G. Fujimoto, "Imaging of macular diseases with optical coherence tomography," Ophthalmology, vol. 102, pp. 217-229, 1995

[5] J. S. Schuman, M. R. Hee, A. V. Arya, T. Pedut-Kloizman, C. A. Puliafito, J. G. Fujimoto, and E. A. Swanson, "Optical coherence tomography: a new tool for glaucoma diagnosis," Current Opinion in Ophthalmology, vol. 6, pp. 89-95, 1995

[6] J. S. Schuman, M. R. Hee, C. A. Puliafito, C. Wong, T. Pedut-Kloizman, C. P. Lin, E. Hertzmark, J. A. Izatt, E. A. Swanson, and J. G. Fujimoto, "Quantification of nerve fiber layer thickness in normal and glaucomatous eyes using optical coherence tomography," Archives of Ophthalmology, vol. 113, pp. 586-596, 1995

[7] J. S. Schuman, T. PedutKloizman, L. Pieroth, E. Hertzmark, M. R. Hee, J. R. Wilkins, J. G. Coker, C. A. Puliafito, J. G. Fujimoto, and E. A. Swanson, "Quantitation of nerve fiber layer thickness loss over time in the glaucomatous monkey model using optical coherence tomography," Investigative Ophthalmology \& Visual Science, vol. 37, pp. 5255-5255, 1996

[8] M. R. Hee, C. A. Puliafito, J. S. Duker, E. Reichel, J. G. Coker, J. R. Wilkins, J. S. Schuman, E. A. Swanson, and J. G. Fujimoto, "Topography of diabetic macular edema with optical coherence tomography," Ophthalmology, vol. 105, pp. 360-370, 1998

[9] M. A. Choma, M. V. Sarunic, C. H. Yang, and J. A. Izatt, "Sensitivity advantage of swept source and Fourier domain optical coherence tomography," Optics Express, vol. 11, pp. 2183-2189, 2003

[10] J. F. de Boer, B. Cense, B. H. Park, M. C. Pierce, G. J. Tearney, and B. E. Bouma, "Improved signal-to-noise ratio in spectral-domain compared with time-domain optical coherence tomography," Optics Letters, vol. 28, pp. 2067-2069, 2003

[11] A. F. Fercher, C. K. Hitzenberger, G. Kamp, and S. Y. Elzaiat, "Measurement of Intraocular Distances by Backscattering Spectral Interferometry," Optics Communications, vol. 117, pp. 43-48, 1995

[12] R. Leitgeb, C. K. Hitzenberger, and A. F. Fercher, "Performance of Fourier domain vs. time domain optical coherence tomography," Optics Express, vol. 11, pp. 889-894, 2003

[13] R. A. Leitgeb, W. Drexler, A. Unterhuber, B. Hermann, T. Bajraszewski, T. Le, A. Stingl, and A. F. Fercher, "Ultrahigh resolution Fourier domain optical coherence tomography," Optics Express, vol. 12, pp. 2156-2165, 2004

[14] N. Nassif, B. Cense, B. H. Park, S. H. Yun, T. C. Chen, B. E. Bouma, G. J. Tearney, and J. F. de Boer, "In vivo human retinal imaging by ultrahigh-speed spectral domain optical coherence tomography," Opt Lett, vol. 29, pp. 480-2, 2004

[15] N. A. Nassif, B. Cense, B. H. Park, M. C. Pierce, S. H. Yun, B. E. Bouma, G. J. Tearney, T. C. Chen, and J. F. de Boer, "In vivo high-resolution video-rate spectral-domain optical coherence tomography of the human retina and optic nerve," Optics Express, vol. 12, pp. 367-376, 2004

[16] M. Wojtkowski, T. Bajraszewski, I. Gorczynska, P. Targowski, A. Kowalczyk, W. Wasilewski, and C. Radzewicz, "Ophthalmic imaging by spectral optical coherence tomography," Am J Ophthalmol, vol. 138, pp. 412-9, 2004 
[17] M. Wojtkowski, R. Leitgeb, A. Kowalczyk, T. Bajraszewski, and A. F. Fercher, "In vivo human retinal imaging by Fourier domain optical coherence tomography," Journal of Biomedical Optics, vol. 7, pp. 457-463, 2002

[18] M. Wojtkowski, V. Srinivasan, J. G. Fujimoto, T. Ko, J. S. Schuman, A. Kowalczyk, and J. S. Duker, "Three-dimensional retinal imaging with high-speed ultrahigh-resolution optical coherence tomography," Ophthalmology, vol. 112, pp. 1734-46, 2005

[19] S. Jiao, R. Knighton, X. Huang, G. Gregori, and C. A. Puliafito, "Simultaneous acquisition of sectional and fundus ophthalmic images with spectral-domain optical coherence tomography," Optics Express, vol. 13, 2005

[20] U. Schmidt-Erfurth, R. A. Leitgeb, S. Michels, B. Povazay, S. Sacu, B. Hermann, C. Ahlers, H. Sattmann, C. Scholda, A. F. Fercher, and W. Drexler, "Three-dimensional ultrahigh-resolution optical coherence tomography of macular diseases," Invest Ophthalmol Vis Sci, vol. 46, pp. 3393402, 2005

[21] G. Hausler and M. W. Linduer, "'Coherence radar" and "spectral radar"-new tools for dermatological diagnosis," Journal of Biomedical Optics, vol. 3, pp. 21-31, 1998

[22] M. Wojtkowski, T. Bajraszewski, P. Targowski, and A. Kowalczyk, "Real-time in vivo imaging by high-speed spectral optical coherence tomography," Optics Letters, vol. 28, pp. 1745-1747, 2003

[23] S. R. Chinn, E. A. Swanson, and J. G. Fujimoto, "Optical coherence tomography using a frequency-tunable optical source," Optics Letters, vol. 22, pp. 340-342, 1997

[24] B. Golubovic, B. E. Bouma, G. J. Tearney, and J. G. Fujimoto, "Optical frequency-domain reflectometry using rapid wavelength tuning of a $\mathrm{Cr} 4+$ :forsterite laser," Optics Letters, vol. 22, pp. 1704-1706, 1997

[25] R. Huber, M. Wojtkowski, K. Taira, J. G. Fujimoto, and K. Hsu, "Amplified, frequency swept lasers for frequency domain reflectometry and OCT imaging: design and scaling principles," Optics Express, vol. 13, pp. 3513-3528, 2005

[26] S. H. Yun, C. Boudoux, G. J. Tearney, and B. E. Bouma, "High-speed wavelength-swept semiconductor laser with a polygon-scanner-based wavelength filter," Optics Letters, vol. 28, pp. 1981-1983, 2003

[27] V. J. Srinivasan, M. Wojtkowski, A. J. Witkin, J. S. Duker, T. H. Ko, M. Carvalho, J. S. Schuman, A. Kowalczyk, and J. G. Fujimoto, "High-definition and 3-dimensional imaging of macular pathologies with high-speed ultrahigh-resolution optical coherence tomography," Ophthalmology, vol. 113, pp. 2054-2065, 2006

[28] V. Christopoulos, L. Kagemann, G. Wollstein, H. R. Ishikawa, M. L. Gabriele, M. Wojtkowski, V. Srinivasan, J. G. Fujimoto, J. S. Duker, D. K. Dhaliwal, and J. S. Schuman, "In vivo corneal highspeed, ultra-high-resolution optical coherence tomography," Archives of Ophthalmology, vol. 125, pp. $1027-1035,2007$

[29] A. J. Witkin, M. Wojtkowski, E. Reichel, V. J. Srinivasan, J. G. Fujimoto, J. S. Schuman, and J. S. Duker, "Photoreceptor disruption secondary to posterior vitreous detachment as visualized using high-speed ultrahigh-Resolution optical coherence tomography," Archives of Ophthalmology, vol. 125, pp. 1579-1580, 2007

[30] M. K. Yoon, R. W. Chen, T. R. Hedges, V. J. Srinivasan, W. Gorczynska, J. G. Fujimoto, M. Wojtkowski, J. S. Schuman, and J. S. Duker, "High-speed, Ultrahigh resolution optical coherence tomography of the retina in hunter syndrome," Ophthalmic Surgery Lasers \& Imaging, vol. 38, pp. 423-428, 2007

[31] M. L. Gabriele, H. Ishikawa, G. Wollstein, R. A. Bilonick, K. A. Townsend, L. Kagemann, M. Wojtkowski, V. J. Srinivasan, J. G. Fujimoto, J. S. Duker, and J. S. Schuman, "Optical coherence tomography scan circle location and mean retinal nerve fiber layer measurement variability," Invest Ophthalmol Vis Sci, vol. 49, pp. 2315-21, 2008

[32] T. Mumcuoglu, G. Wollstein, M. Wojtkowski, L. Kagemann, H. Ishikawa, M. L. Gabriele, V. Srinivasan, J. G. Fujimoto, J. S. Duker, and J. S. Schuman, "Improved visualization of glaucomatous retinal damage using high-speed ultrahigh-resolution optical coherence tomography," Ophthalmology, vol. 115, pp. 782-789 e2, 2008

[33] V. J. Srinivasan, B. K. Monson, M. Wojtkowski, R. A. Bilonick, I. Gorczynska, R. Chen, J. S. Duker, J. S. Schuman, and J. G. Fujimoto, "Characterization of outer retinal morphology with high-speed, ultrahigh-resolution optical coherence tomography," Invest Ophthalmol Vis Sci, vol. 49, pp. 15719, 2008 
[34] I. Gorczynska, V. J. Srinivasan, L. N. Vuong, R. W. S. Chen, J. J. Liu, E. Reichel, M. Wojtkowski, J. S. Schuman, J. S. Duker, and J. G. Fujimoto, "Projection OCT fundus imaging for visualising outer retinal pathology in non-exudative age-related macular degeneration," British Journal of Ophthalmology, vol. 93, pp. 603-609, 2009

[35] W. Drexler, U. Morgner, R. K. Ghanta, F. X. Kärtner, J. S. Schuman, and J. G. Fujimoto, "Ultrahigh-resolution ophthalmic optical coherence tomography," Nature Medicine, vol. 7, pp. 502507, 2001

[36] S. H. Yun, G. J. Tearney, J. F. de Boer, N. Iftimia, and B. E. Bouma, "High-speed optical frequency-domain imaging," Optics Express, vol. 11, pp. 2953-2963, 2003

[37] M. Wojtkowski, V. J. Srinivasan, T. H. Ko, J. G. Fujimoto, A. Kowalczyk, and J. S. Duker, "Ultrahigh-resolution, high-speed, Fourier domain optical coherence tomography and methods for dispersion compensation," Optics Express, vol. 12, pp. 2404-2422, 2004

[38] B. Cense, N. Nassif, T. C. Chen, M. C. Pierce, S. Yun, B. H. Park, B. Bouma, G. Tearney, and J. F. de Boer, "Ultrahigh-resolution high-speed retinal imaging using spectral-domain optical coherence tomography," Optics Express, vol. 12, pp. 2435-2447, 2004

[39] B. Potsaid, I. Gorczynska, V. J. Srinivasan, Y. L. Chen, J. Jiang, A. Cable, and J. G. Fujimoto, "Ultrahigh speed Spectral/Fourier domain OCT ophthalmic imaging at 70,000 to 312,500 axial scans per second," Optics Express, vol. 16, pp. 15149-15169, 2008

[40] Y. Yasuno, Y. J. Hong, S. Makita, M. Yamanari, M. Akiba, M. Miura, and T. Yatagai, "In vivo highcontrast imaging of deep posterior eye by 1-mu m swept source optical coherence tomography and scattering optical coherence angiography," Optics Express, vol. 15, pp. 6121-6139, 2007

[41] B. Povazay, B. Hofer, C. Torti, B. Hermann, A. R. Tumlinson, M. Esmaeelpour, C. A. Egan, A. C. Bird, and W. Drexler, "Impact of enhanced resolution, speed and penetration on threedimensional retinal optical coherence tomography," Optics Express, vol. 17, pp. 4134-4150, 2009

[42] J. A. Izatt, M. D. Kulkami, S. Yazdanfar, J. K. Barton, and A. J. Welch, "In vivo bidirectional color Doppler flow imaging of picoliter blood volumes using optical coherence tomography," Optics Letters, vol. 22, pp. 1439-41, 1997

[43] R. A. Leitgeb, L. Schmetterer, W. Drexler, A. F. Fercher, R. J. Zawadzki, and T. Bajraszewski, "Real-time assessment of retinal blood flow with ultrafast acquisition by color Doppler Fourier domain optical coherence tomography," Optics Express, vol. 11, pp. 3116-3121, 2003

[44] B. R. White, M. C. Pierce, N. Nassif, B. Cense, B. H. Park, G. J. Tearney, B. E. Bouma, T. C. Chen, and J. F. de Boer, "In vivo dynamic human retinal blood flow imaging using ultra-highspeed spectral domain optical Doppler tomography," Optics Express, vol. 11, pp. 3490-3497, 2003

[45] Z. Chen, T. E. Milner, D. Dave, and J. S. Nelson, "Optical Doppler tomographic imaging of fluid flow velocity in highly scattering media," Optics Letters, vol. 22, pp. 64-6, 1997

[46] S. Makita, Y. Hong, M. Yamanari, T. Yatagai, and Y. Yasuno, "Optical coherence angiography," Optics Express, vol. 14, pp. 7821-7840, 2006

[47] A. Szkulmowska, M. Szkulmowski, D. Szlag, A. Kowalczyk, and M. Wojtkowski, "Threedimensional quantitative imaging of retinal and choroidal blood flow velocity using joint Spectral and Time domain Optical Coherence Tomography," Optics Express, vol. 17, pp. 10584-10598, 2009

[48] S. Makita, T. Fabritius, and Y. Yasuno, "Quantitative retinal-blood flow measurement with threedimensional vessel geometry determination using ultrahigh-resolution Doppler optical coherence angiography," Optics Letters, vol. 33, pp. 836-838, 2008

[49] R. K. Wang, S. L. Jacques, Z. Ma, S. Hurst, S. R. Hanson, and A. Gruber, "Three dimensional optical angiography," Optics Express, vol. 15, pp. 4083-4097, 2007

[50] R. M. Werkmeister, N. Dragostinoff, M. Pircher, E. Gotzinger, C. K. Hitzenberger, R. A. Leitgeb, and L. Schmetterer, "Bidirectional Doppler Fourier-domain optical coherence tomography for measurement of absolute flow velocities in human retinal vessels," Optics Letters, vol. 33, pp. 2967-2969, 2008

[51] V. J. Srinivasan, S. Sakadzic, I. Gorczynska, S. Ruvinskaya, W. C. Wu, J. G. Fujimoto, and D. A. Boas, "Quantitative cerebral blood flow with Optical Coherence Tomography," Optics Express, vol. 18, pp. 2477-2494, 2010 
[52] B. Potsaid, B. Baumann, D. Huang, S. Barry, A. E. Cable, J. S. Schuman, J. S. Duker, and J. G. Fujimoto, "Ultrahigh speed 1050nm swept source / Fourier domain OCT retinal and anterior segment imaging at 100,000 to 400,000 axial scans per second," Optics Express, vol. 18, pp. 20029-20048, 2010

[53] B. Povazay, K. Bizheva, B. Hermann, A. Unterhuber, H. Sattmann, A. F. Fercher, W. Drexler, C. Schubert, P. K. Ahnelt, M. Mei, R. Holzwarth, W. J. Wadsworth, J. C. Knight, and P. S. Russel, "Enhanced visualization of choroidal vessels using ultrahigh resolution ophthalmic OCT at 1050 nm," Optics Express, vol. 11, pp. 1980-1986, 2003

[54] A. Unterhuber, B. Povazay, B. Hermann, H. Sattmann, A. Chavez-Pirson, and W. Drexler, "In vivo retinal optical coherence tomography at $1040 \mathrm{~nm}$-enhanced penetration into the choroid," Optics Express, vol. 13, pp. 3252-3258, 2005

[55] S. H. Yun, G. J. Tearney, J. F. de Boer, and B. E. Bouma, "Motion artifacts in optical coherence tomography with frequency-domain ranging," Optics Express, vol. 12, pp. 2977-2998, 2004

[56] J. Walther, A. Kruger, M. Cuevas, and E. Koch, "Effects of axial, transverse, and oblique sample motion in FD OCT in systems with global or rolling shutter line detector," Journal of the Optical Society of America a-Optics Image Science and Vision, vol. 25, pp. 2791-2802, 2008

[57] V. J. Srinivasan, T. H. Ko, M. Wojtkowski, M. Carvalho, A. Clermont, S. E. Bursell, Q. H. Song, J. Lem, J. S. Duker, J. S. Schuman, and J. G. Fujimoto, "Noninvasive volumetric Imaging and morphometry of the rodent retina with high-speed, ultrahigh-resolution optical coherence tomography," Investigative Ophthalmology \& Visual Science, vol. 47, pp. 5522-5528, 2006

[58] R. A. Leitgeb, L. Schmetterer, C. K. Hitzenberger, A. F. Fercher, F. Berisha, M. Wojtkowski, and T. Bajraszewski, "Real-time measurement of in vitro flow by Fourier-domain color Doppler optical coherence tomography," Opt Lett, vol. 29, pp. 171-3, 2004

[59] A. C. Sull, L. N. Vuong, L. L. Price, V. J. Srinivasan, I. Gorczynska, J. G. Fujimoto, J. S. Schuman, and J. S. Duker, "Comparison of spectral/Fourier domain optical coherence tomography instruments for assessment of normal macular thickness," Retina, vol. 30, pp. 235-45, 2010

[60] J. Ho, A. C. Sull, L. N. Vuong, Y. Chen, J. Liu, J. G. Fujimoto, J. S. Schuman, and J. S. Duker, "Assessment of Artifacts and Reproducibility across Spectral- and Time-Domain Optical Coherence Tomography Devices," Ophthalmology, vol. 116, pp. 1960-1970, 2009

[61] J. Ho, D. P. E. Castro, L. C. Castro, Y. L. Chen, J. Liu, C. Mattox, C. Krishnan, J. G. Fujimoto, J. S. Schuman, and J. S. Duker, "Clinical Assessment of Mirror Artifacts in Spectral-Domain Optical Coherence Tomography," Investigative Ophthalmology \& Visual Science, vol. 51, pp. 3714-3720, 2010

[62] J. S. Kim, H. Ishikawa, M. L. Gabriele, G. Wollstein, R. A. Bilonick, L. Kagemann, J. G. Fujimoto, and J. S. Schuman, "Retinal Nerve Fiber Layer Thickness Measurement Comparability between Time Domain Optical Coherence Tomography (OCT) and Spectral Domain OCT," Investigative Ophthalmology \& Visual Science, vol. 51, pp. 896-902, 2010

[63] V. Manjunath, M. Taha, J. G. Fujimoto, and J. S. Duker, "Choroidal thickness in normal eyes measured using Cirrus HD optical coherence tomography," Am J Ophthalmol, vol. 150, pp. 325329 e1, 2010 


\section{Motion Correction and Image Enhancement}

\section{Sponsors}

National Institutes of Health-RO1-EY11289-23, R01-EY13178-07, P30-EY008098

Air Force Office of Scientific Research-FA9550-07-1-0101 and FA9550-07-1-0014

German Research Foundation-DFG-GSC80-SAOT

\section{Project Staff}

Prof. James G. Fujimoto, Dr. Bernhard Baumann, Dr. Ben Potsaid, Jonathan J. Liu (MIT)

Prof. Joachim Hornegger, Martin Kraus (University of Erlangen, Germany)

Recently, the development of high speed OCT [1] has made it possible to capture densely sampled 3D volume data in a matter of seconds. These volumetric data sets are in principle able to show a more complete view of tissue morphology and pathology, compared to 2D cross-sectional images that are traditionally used in ophthalmology and other areas. However, OCT image acquisition still requires a finite time and a $3 \mathrm{D}$ volumetric data set is composed from a 2D grid of many sequentially acquired axial scan profiles. For example, capturing a volumetric data set composed of $300 \times 300$ axial scans on an OCT device running at about $50 \mathrm{kHz}$ axial scan rate requires about two seconds. During acquisition, relative motion between the OCT device and the object produces motion artifacts. The unknown motion causes the OCT beam not to be sampling at the expected area, leading to areas of the object that are scanned multiple times while others are not covered at all. In ophthalmologic OCT [2], several sources of patient motion such as respiration and changes in fixation are unavoidable and cause artifacts. These artifacts degrade image quality and limit the usability of 3D OCT scans for quantitative measurements [3]. Most previous methods to correct motion require a motion-free reference, attempt to measure the motion [4] or make simplifying assumptions on the motion artifact problem [5]. Many approaches lack the ability to correct motion in all three dimensions.
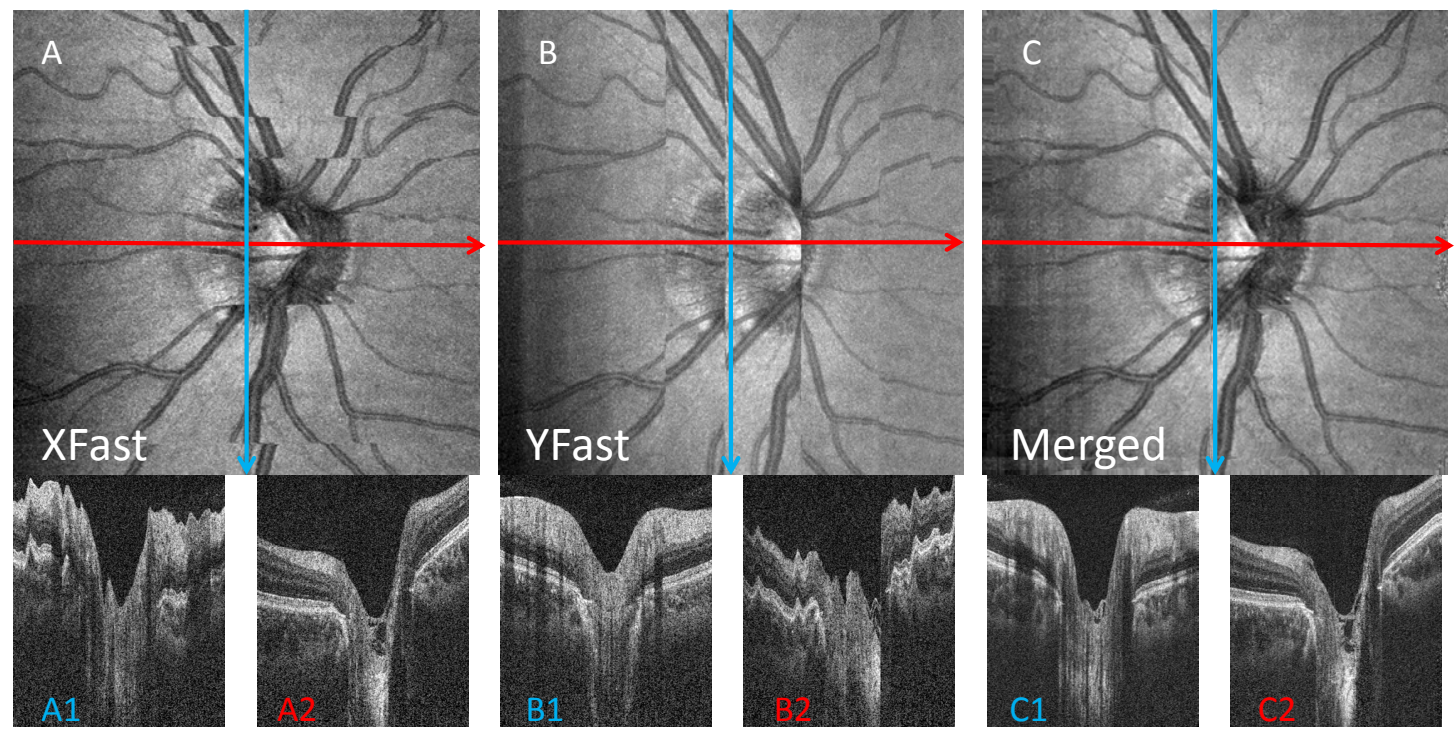

Figure 3.1. A,B: En face projections of input OCT volumes of the optic nerve head of a healthy subject with orthogonal fast scan axes captured with a $1050 \mathrm{~nm}$ spectral/Fourier domain system at $47 \mathrm{kHz}$. 400x400 A-Scans were acquired in $\sim 4$ seconds each. Significant motion artifacts because of saccadic changes of the subject's fixation can be seen which completely destroy the topology of the object. C: En face projection of registered and merged volume generated from $A$ and $B$. Lower row: Cross-sectional slices of respective volumes at positions marked by the red and blue arrows in the en face views. No motion artifacts can be seen in C, as opposed to A and B. Significant signal to noise ratio improvements are achieved by merging volumes. 
Working in cooperation with the Pattern Recognition Lab at the University of Erlangen, Germany, we have developed a purely software based motion correction algorithm that utilizes two or more input OCT with different scanning patterns. The scan patterns differ by which direction of the imaging volume is scanned first. Depending on the scan pattern, the data is always comparatively unaffected by motion in this fast scan direction, while the orthogonal direction suffers from much more severe motion artifacts. This is because in the fast direction, motion is effectively almost absent because of the rapid acquisition time. When two volumes with orthogonal fast scan directions are acquired, the fast direction in one volume is the slow one in the other and vice versa. This complementary dependence can be used to correct motion artifacts in both volumes at the same time. No motion free reference image such as a fundus photograph is needed.

A global objective function is optimized to estimate a dense displacement field for each volume, modeling object motion during the scans. In the objective function we try to find the unknown motion so that after correction the similarity between the volumes is maximized. In addition, prior knowledge about the nature of the motion is incorporated. After correction, a final combined volume is constructed as a sum of the individual motion corrected volumes. This method significantly enhances contrast and signal quality of the OCT images. The weighting also takes into account the possibility that certain areas of the tissue might not have been scanned in one or more of the input volumes.

Datasets of the macula and optic disc from healthy and pathological eyes were recorded on multiple OCT systems with different acquisition speeds. Results (see Figure 3.1 and Figure 3.2) show that the algorithm is able to correct motion in all three dimensions. Both slow drift as well as saccadic movement are corrected. Quantitative assessment of registration performance and visual inspection indicates very good registration results. The resulting volumes do not show visible motion artifacts. In addition, the signal to noise ratio of the final volume is visibly superior to the input volumes. Using a graphics card for acceleration, the process takes as little as 25 seconds for two $200 \times 200$ volumes.

This method is a robust, software based, motion artifact correction solution which can remove distortion introduced by movement in all three dimensions. These methods promise to facilitate the acquisition of large 3D-OCT data sets and improve measurement reproducibility without the need for active eye tracking or motion free reference images. In addition, subsequent processing image processing steps such as segmentation should profit from the increased signal quality.
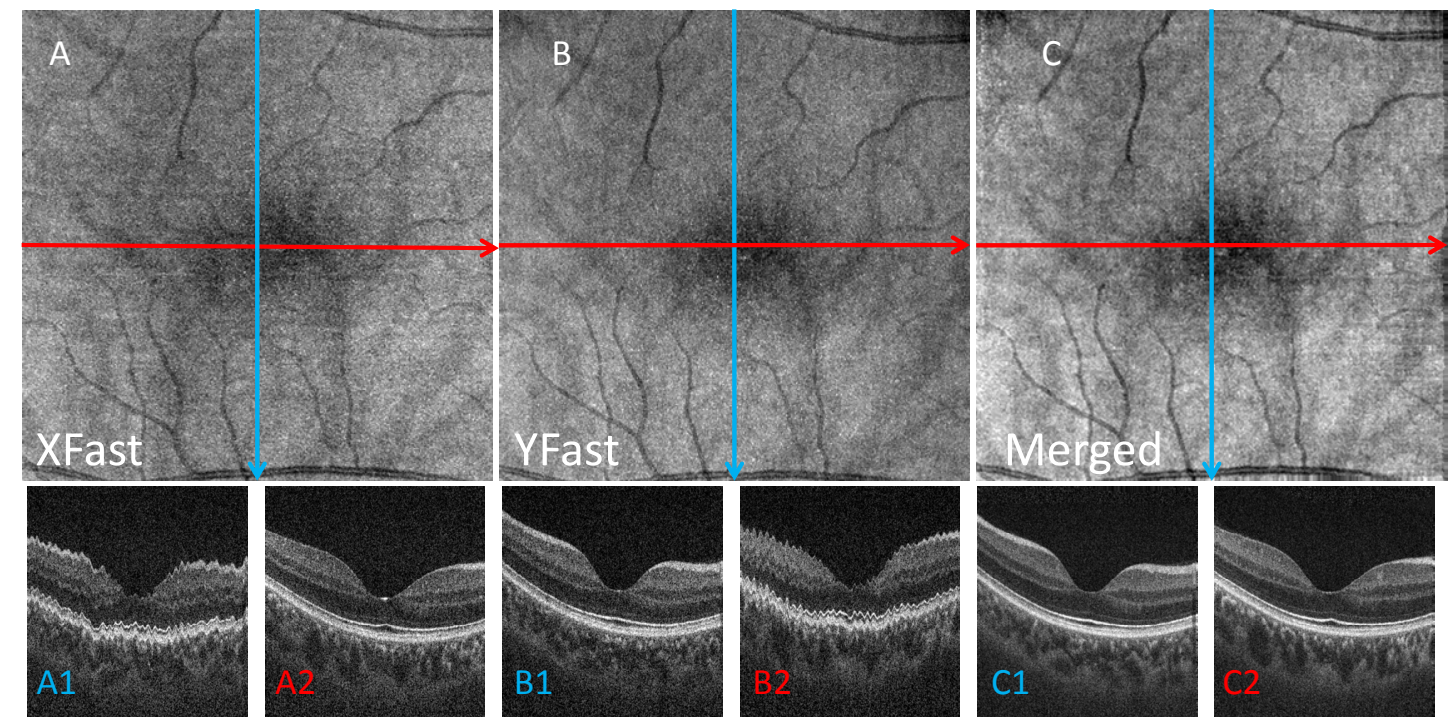

Figure 3.2. A,B: En face projections of input OCT volumes of macular region of a healthy subject with orthogonal fast scan axis. System and scan parameters are the same as in Fig. 1. As can be seen in A1 and B2, axial motion distorts the image in the slow scan direction. $\mathrm{C} 1$ and $\mathrm{C} 2$ show no motion artifacts and improved signal quality. Comparing $A$ and $B$ with $C$ the en face projection of the registered and merged result also improves definition and contrast. 


\section{References}

[1] D. Huang, E. A. Swanson, C. P. Lin, J. S. Schuman, W. G. Stinson, W. Chang, M. R. Hee, T. Flotte, K. Gregory, C. A. Puliafito, and J. G. Fujimoto, "Optical Coherence Tomography," Science, vol. 254, pp. 1178-1181, Nov 221991.

[2] J. S. Schuman, C. A. Puliafito, and J. G. Fujimoto, Optical coherence tomography of ocular diseases, 2nd edition. Thorofare, NJ: Slack Inc., 2004.

[3] L. A. Paunescu, J. S. Schuman, L. L. Price, P. C. Stark, S. Beaton, H. Ishikawa, G. Wollstein, and J. G. Fujimoto, "Reproducibility of nerve fiber thickness, macular thickness, and optic nerve head measurements using StratusOCT," Investigative Ophthalmology and Visual Science, vol. 45, pp. 1716-1724, Jun 2004.

[4] R. D. Ferguson, D. X. Hammer, L. A. Paunescu, S. Beaton, and J. S. Schuman, "Tracking optical coherence tomography," Optics Letters, vol. 29, pp. 2139-2141, SEP 152004.

[5] B. Potsaid, I. Gorczynska, V. J. Srinivasan, Y. L. Chen, J. Jiang, A. Cable, and J. G. Fujimoto, "Ultrahigh speed Spectral/Fourier domain OCT ophthalmic imaging at 70,000 to 312,500 axial scans per second," Optics Express, vol. 16, pp. 15149-15169, 2008. 


\section{High Speed Three-Dimensional OCT Endoscopic Imaging}

\section{Sponsors}

National Institutes of Health - R01-CA075289-13 and R01-EY11289-24

Air Force Office of Scientific Research - FA9550-07-1-0101, FA9550-040-07-1-0014

Center for Integration of Medicine and Innovation Technology (CIMIT)

\section{Project Staff}

Prof. James G. Fujimoto, Dr. Chao Zhou, Hsiang-Chieh Lee, Tsung-Han Tsai (MIT)

Dr. Hiroshi Mashimo, M.D., Ph.D. (VA Medical Center)

Dr. Joseph Schmitt, Dr. Desmond Adler (LightLab Imaging)

New gastrointestinal $(\mathrm{Gl})$ cancers will be found in more than 274,330 patients and will cause more than 139,580 deaths in the United States in 2010 [1]. When detected and treated early, the 5-year survival rate for colorectal cancer increases by a factor of 1.4 [1]. For esophageal cancer, the rate increases by a factor of 2 [1]. Unfortunately, many early-stage lesions are missed during standard endoscopic examination. Approximately $15-27 \%$ of small $(<5 \mathrm{~mm}$ ) lesions in the colon go undetected, with rates of flat lesions missed being $\sim 2 x$ worse than polypoid lesions [2]. The situation is worse in the esophagus, where $30-40 \%$ of relatively large, invasive cancers are missed [3]. An early cancer detection method for imaging the GI tract may reduce morbidity and mortality associated with the disease, thereby allowing physicians to intervene earlier using minimally invasive therapies.

Gastroenterology is an especially relevant application for OCT because of the high incidence rates of GI pathologies, the clinical benefits of early detection, and the need for pre- and post-treatment assessment of therapies. OCT imaging can be performed using fiber optics and miniaturized lens systems, enabling endoscopic OCT inside the human body in conjunction with conventional video endoscopy. An OCT probe can be inserted through the working channel of a standard endoscope, thus enabling two-dimensional (2D), depth-resolved imaging of tissue microstructure ranging over a linear dimension of several millimeters to several centimeters with micron-scale resolution [4].

The majority of $\mathrm{Gl}$ cancers begin as small ( $<100 \mathrm{um}$ ) lesions that are difficult to identify with conventional endoscopy. OCT is well-suited for detecting the changes in tissue microstructure associated with early $\mathrm{Gl}$ cancers. Since the lesions are not visually apparent, however, it is necessary to survey a relatively large area of the GI tract with high-resolution OCT in order to detect the lesions. Tissue motion is a limiting factor in the Gl tract, therefore in vivo imaging must be performed at extremely high speeds. Recent advances in ultrahigh speed OCT using Fourier domain detection techniques [5-7] have enabled in vivo 3D-OCT with imaging speeds of up to 370,000 axial lines per second [8]. By developing miniaturized probes that scan in two spatial dimensions, endoscopic 3D-OCT can be performed. 3D-OCT overcomes the limitations of 2D analysis by enabling comprehensive visualization of tissue microstructure over larger fields of view. Volumetric data sets enable the generation of en face images, thus providing precise registration of cross-sectional data with en face features. Projection techniques can be used to selectively display specific depths within the tissue, thereby enhancing image contrast for specific architectural features. Cross-sectional images with arbitration orientation can also be generated from the 3D data.

Our group has developed an endoscopic imaging system that uses a Fourier domain modelocked (FDML) laser, a state-of-the-art data acquisition system, and a rapid, spiral-scanning fiberoptic endoscope probe [9-12]. This "3D endomicroscopy" system is capable of acquiring 3D-OCT data at unprecedented speeds and at 3D resolutions, thus enabling the detection of small $\mathrm{GI}$ structures that have been linked to Barrett's esophagus and colon cancer. Three-dimensional acquisition also enables a variety of powerful visualization techniques, such as frame averaging for speckle reduction, en face image generation for comparison to microscopy or endoscopy, and the assessment of ablation therapies in the GI tract.

Figure 4.1 and Figure 4.2 shows a schematic of this system and the fiberoptic probe. The system was developed in collaboration with LightLab Imaging and uses swept source / Fourier domain OCT detection to achieve fast imaging speeds and high sensitivity. An FDML laser with a center wavelength of $1310 \mathrm{~nm}$, a total tuning range of $180 \mathrm{~nm}$, a full-width-half-maximum bandwidth of $122 \mathrm{~nm}$, and an average output 
power of $42 \mathrm{~mW}$ at a sweep rate of $60 \mathrm{kHz}$ is used as the swept light source. Another version of this endoscopic OCT imaging system with a sweep rate of $100 \mathrm{kHz}$ was reported in Nature Photonics and demonstrated for in vivo animal imaging [9]. The current clinical system uses a lower imaging speed in order to improve the sensitivity and imaging range for human tissue. The laser supports an axial resolution of $6.5 \mathrm{um}$ in air ( $\sim$ um in tissue). The maximum imaging depth is $1.5 \mathrm{~mm}$ in tissue. System sensitivity with $13 \mathrm{~mW}$ of power incident on the sample is $105 \mathrm{~dB}$ at short delays, and it drops by $7.8 \mathrm{~dB}$ at a ranging depth of $2 \mathrm{~mm}$ in air. The light exposure level is within the guidelines established in ANSI standards and is consistent with optical power levels used in commercial endoscopic and cardiovascular OCT instruments.

Volumetric data is acquired at 60,000 axial lines per second and 70 frames per second by using a rotary fiberoptic probe. The probe is a spiral-scanning device that combines rapid rotary motion (up to $80 \mathrm{~Hz}$ rotation) with a linear pullback $(0.5-5 \mathrm{~mm} / \mathrm{sec})$ to image $3 \mathrm{D}$ volumes. The optical fiber is placed inside a flexible polymer tube, which is necessary for mechanical stability. The tube can be water flushed using a syringe located outside the body in order to reduce specular reflection from the tissue surface and to wash debris from the imaging site. The proximal end of the fiber is attached to a patient interface unit (PIU) containing the rotary and push/pull actuators. The distal end of the fiber is attached to an angle-polished microlens and provides a 9 um spot diameter. The total probe diameter is $2.5 \mathrm{~mm}$, which allows it to pass through the working channel of a wide variety of commercial endoscopes.

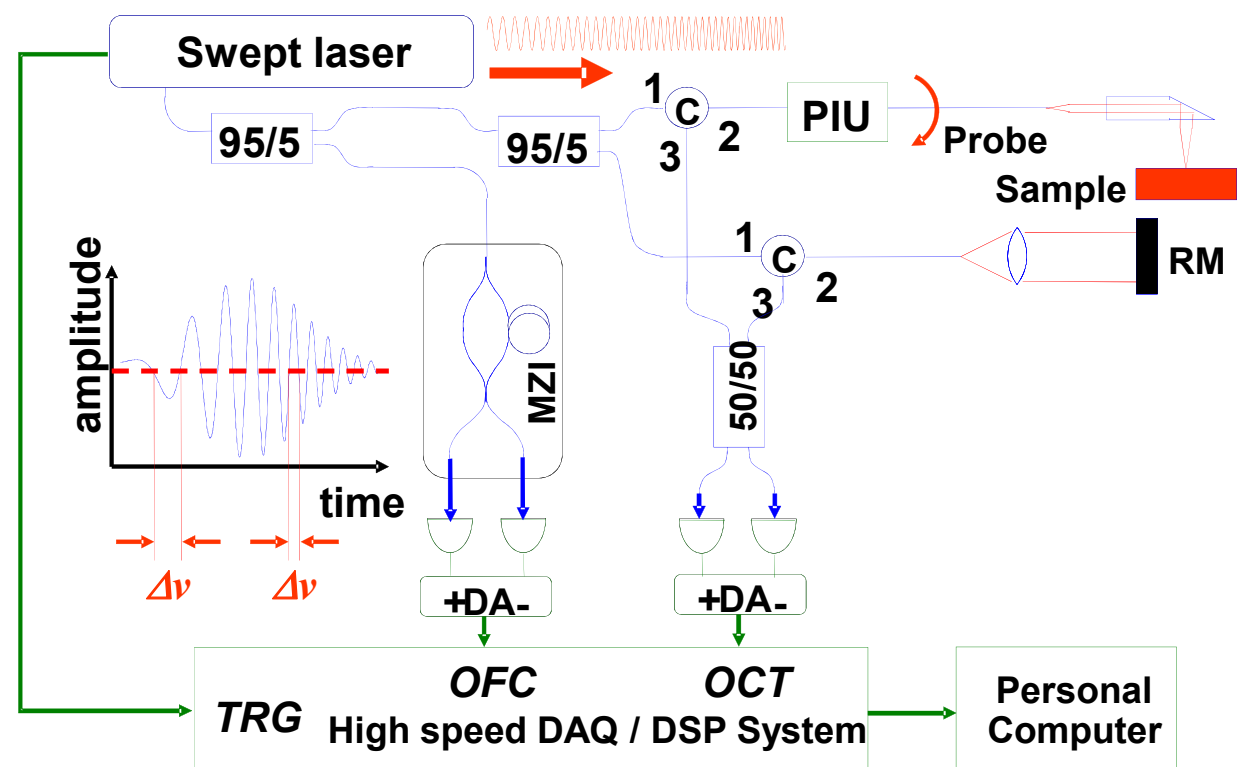

Figure 4.1. OCT system for ultrahigh speed endoscopic 3D-OCT imaging. The system uses swept source / Fourier domain OCT with a high speed Fourier domain modelocked (FDML) laser. Volumetric data is using a rotary fiberoptic endoscope probe. The probe can be used in the working channel of a standard endoscope.

\subsection{OCT Imaging of the GI Tract}

Endoscopic OCT has been applied extensively for GI studies [13-15] with a particular emphasis on imaging in Barrett's esophagus patients [16-21]. Barrett's esophagus is a condition associated with gastroesophageal reflux disease (GERD) where chronic exposure of the esophagus to stomach acid causes pre-cancerous changes of the esophagus. Conventional endoscopic imaging lacks sensitivity for detecting high grade dysplasia, the immediate precursor to esophageal cancer. Therefore, there is considerable interest in developing new imaging techniques which can detect these changes. Our group works in collaboration with Dr. Hiroshi Mashimo, M.D., Ph.D. and clinicians at the VA Boston Medical Center to perform clinical studies of endoscopic OCT applications. 

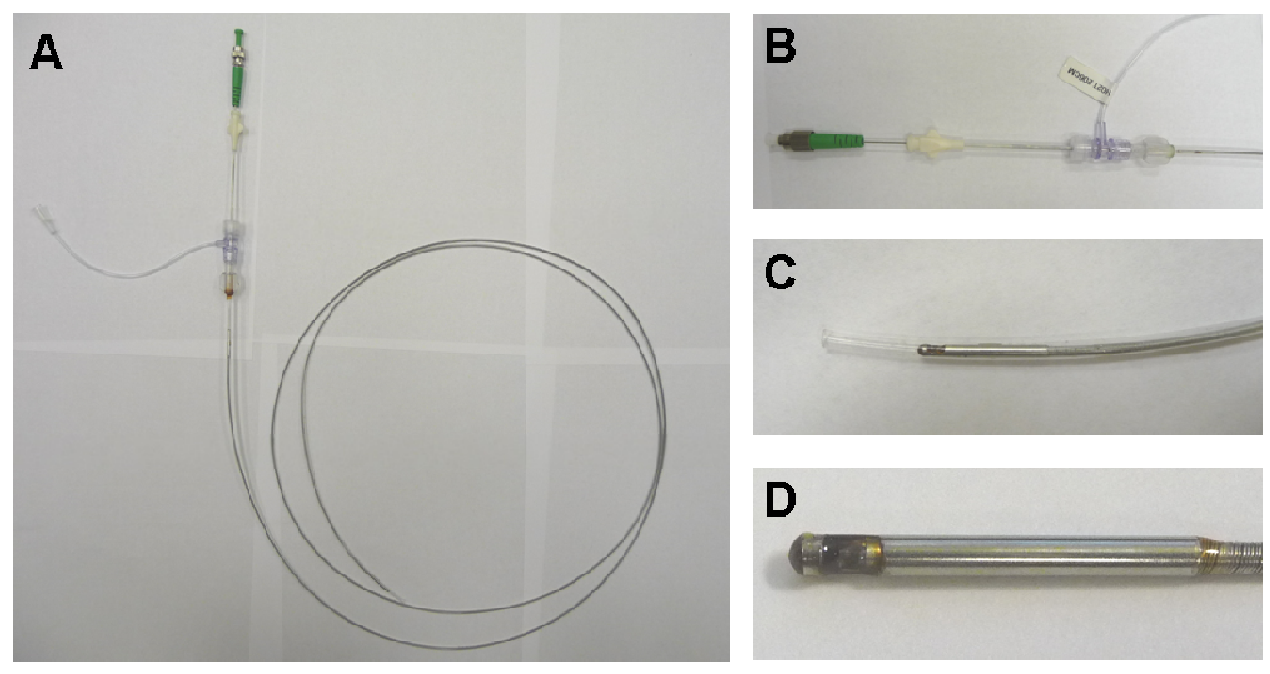

Figure 4.2. (A) Endoscopic OCT imaging probe. (B) Proximal end of the imaging probe. (C) Distal end of the imaging probe. (D) OCT imaging head is sealed by a stainless steel hypotube and epoxy.

Figure 4.3 shows examples of OCT images from a volumetric data set in a patient with Barrett's esophagus. Volumetric data sets enable the generation of en face images, thereby enabling precise registration of cross-sectional data with en face features. Projection techniques can be used to display specific depths within the tissue selectively, thus enhancing image contrast for specific architectural features. Cross-sectional images with arbitration orientation can also be generated from the 3D data. The en face view (Fig. 4.3a) and cross-sectional views (Figs. 4.3b and c) illustrate the characteristic glandular structures associated with Barrett's esophagus, which can be easily distinguished from the normal esophagus which has fine layered structure. The zoomed view of glandular structures (Fig. 4.3d) correlates well with the histology of Barrett's esophagus shown in Fig 4.3e. 3D-OCT endomicroscopy is capable of differentiating glandular and squamous epithelium in vivo. This ability could be important for applications such as the assessment of endoscopic therapies at multiple time points, since it provides an inherent positional registration that is difficult to obtain with cross sectional OCT imaging alone.

Based on previous results from OCT imaging of the human gastrointestinal tract, including histology correlation studies, it is possible to distinguish BE glands from normal glands found near the GEJ or in the esophagus. Figure 4.4 shows OCT images and corresponding histology from an ex vivo endoscopic mucosal resection (EMR) nodule and an in vivo region near the GEJ. Normal esophageal glands in a well circumscribed lobular form are large $(1 \mathrm{~mm})$ with a heterogeneous moderately-scattering center, as shown in Figs. 4.4A and B. BE glands are irregular, smaller (100-300 um), have sharp borders with OCT, and are devoid of signal in their centers, as shown in Figs. 4.4C and D. Gastric glands are found underneath gastric pits and may have slightly more diffuse borders as shown in Figs. 4.4E and $F$.

Our group and collaborators are also performing clinical studies using endoscopic 3D-OCT in the lower gastrointestinal tract. Ulcerative colitis (UC) is a chronic inflammatory condition of the $\mathrm{GI}$ tract that produces abscesses, ulcerations, and bleeding. UC affects up to 780,000 individuals in the United States and Canada and is newly diagnosed in 7,000-46,000 individuals per year [1]. 3D-OCT endomicroscopy images acquired in the rectum near the anal verge are shown in Figure 4.5. The volume is $8 \times 20 \times 1.6 \mathrm{~mm}^{3}$ in dimension and was acquired in 20 seconds. Fig. 4.5a shows an en face image formed by axial summation of a 20 um-thick section centered 350 um beneath the luminal surface. When compared to normal squamous and columnar mucosa, UC tissue appears highly irregular. Large subsurface voids and bands of hyperscattering tissue, possibly fibrotic, are apparent. A wedge of comparatively normal tissue is visible at the right of the image. Fig. $\mathbf{4 . 5 b}$ shows an $X Z$ cross section through the region that reveals a regular, layered architecture consistent with anal squamous mucosa. 


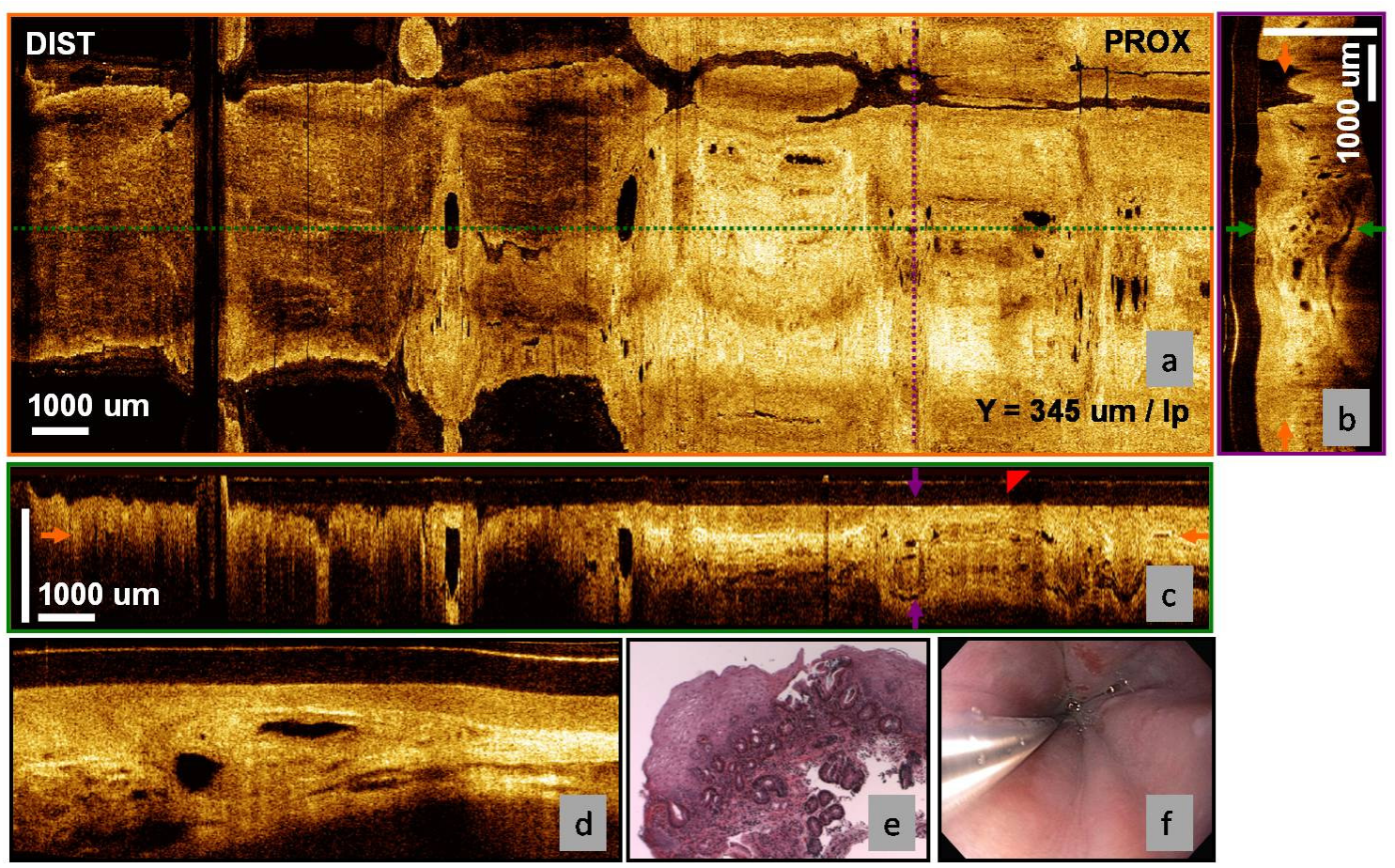

Figure 3.3. 3D-OCT images in Barrett's esophagus. (a) En face image constructed by axial summation of a thin slice ( $20 \mathrm{um}$ ) in the 3D data. (b) $X Z$ cross section shows the characteristic glandular structures associated with Barrett's esophagus. (c) $Y Z$ cross section shows the glandular structures. (d) Close-up view of YZ cross-section. (e) Representative histology of Barrett's esophagus. (f) White-light video endoscopy image of region analyzed with 3D-OCT.

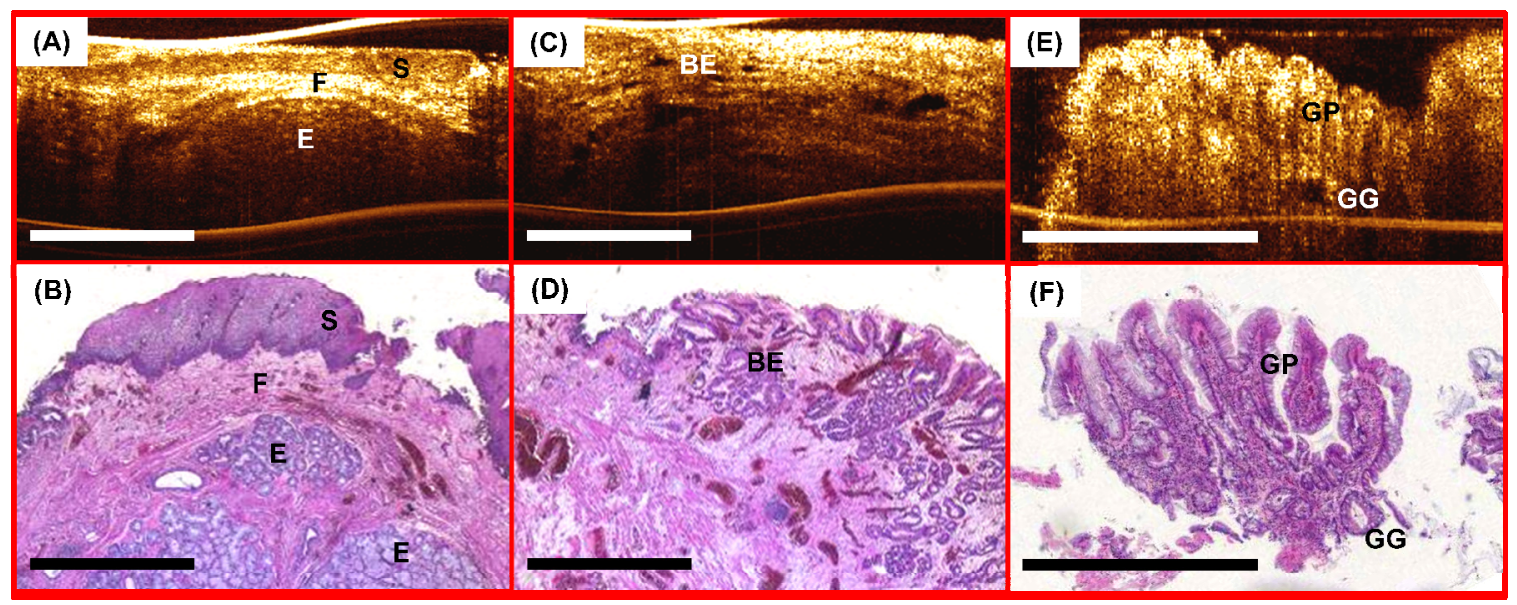

Figure 3.4. OCT images and corresponding histology of esophageal, BE, and gastric glands obtained from an ex vivo esophageal EMR nodule (A-D) and an in vivo image near the GEJ (E-F). $(A, B)$ OCT and corresponding histology demonstrate representative esophageal glands (E) with a diameter of about $1 \mathrm{~mm}$ underneath squamous epithelium (S) and fibrous tissue (F). (C, D) BE glands (BE) were also observed from the same nodule. (E, F) A gastric gland (GG) was observed underneath hyperplastic gastric pits (GP). Scale bars, $1 \mathrm{~mm}$.

The epithelium is thicker when compared to healthy individuals, possibly as a result of healing in response to prior treatment with anti-inflammatory medications. The ulcerated region exhibits a loss of layered or columnar architecture, superficial edema, and a large subsurface abnormality consistent with 
submucosal fibrosis. Fig. 4.5c shows a $Y Z$ cross section with $U C$ and normal squamous tissue visible on the left and right sides of the image, respectively. Fig. 4.5d shows enlarged regions of Fig. 4.5c. Representative histology of UC in glandular mucosa is shown in Fig. 4.5e. Lymphocytic mucosal infiltration is present along with submucosal fibrosis. Ulceration results in the formation of a pseudo-polyp as the epithelium is stripped away to expose the submucosa. Fig. 4.5f shows a white-light video endoscopy image of a patient with active UC. The mucosa appears red, inflamed, and ulcerative. The 3DOCT catheter is also shown in position prior to imaging.
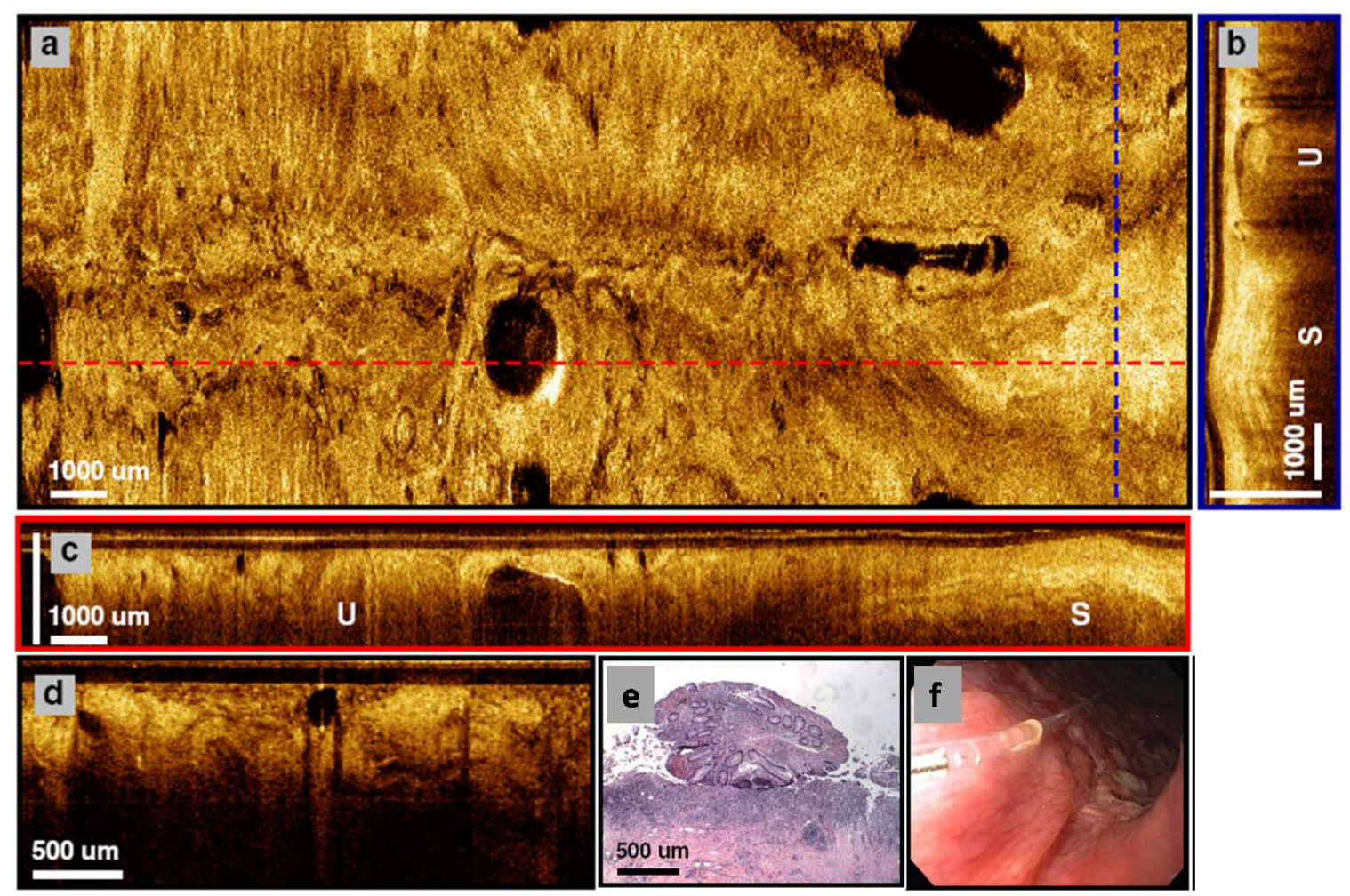

Figure 4.5. 3D-OCT images of ulcerative colitis. (a) En face image constructed by summing a 20 $\mathrm{m}$ axial section. Large, subsurface voids and ulcerations are present, while the regular crypt pattern is absent. Dashed lines show locations of cross-sectional images. (b) $X Z$ cross section contains normal squamous epithelium $S$ and ulcerative colitis $U$. (c) $Y Z$ cross section shows similar structure. (d) Close-up view of left portion of (c) shows disorganized structure and superficial voids. (e) Representative cross-sectional histology of UC shows an ulcerative pseudo-polyp. (f) Whitelight video endoscopy image of UC shows the 3D-OCT probe. The tissue surface is inflamed with ulcerations.

3D-OCT endomicroscopy enables visualization of microstructural differences between the UC and normal regions. Furthermore, the extended field of view allows the evaluation of macro-scale features, such as subsurface voids, as well as microstructural details such as superficial edema and the layered architecture of the normal region.

\subsection{Assessment of Ablation Therapies in the GI Tract}

Ablative therapies, including photodynamic therapy, argon plasma coagulation, radiofrequency ablation (RFA) and cryoablation (CSA) are increasingly performed for diseases in the gastrointestinal (GI) tract such as Barrett's esophagus (BE) [22-25]. Due to the difficulty of immediately taking biopsies from the treated sites, the treatment efficacy typically can be confirmed weeks to months after applying the ablation therapies [24, 26, 27]. Hence, it is important to have real-time evaluation for these endoscopic therapies to further increase the treatment efficacy. Endoscopic three-dimensional optical coherence 
tomography (3D-OCT) is a volumetric imaging technique that is uniquely suited for followup assessment of RFA treatment and was used in this study for evaluating the presence of neosquamous epithelium and subsurface structures over the RFA treated field in the GI tract [9, 10, 28, 29]. In this study, we sought to assess whether esophageal tissues present differences in architectural changes following these different therapies using endoscopic three-dimensional OCT (3D-OCT).

3D-OCT imaging was performed on patients following RFA $(n=10)$ and CSA $(n=2)$ treatments for BE with the Barrx HALO90 system and the CSA Medical systems, respectively. The patients in this study were recruited from volunteers aged from 30 to 80 undergoing diagnostic endoscopy and biopsy for Barrett's esophagus in Boston Veterans Affairs Health System based on a history of a biopsy positive for moderate grade dysplasia within the previous 18 month period.

For the RFA treatment, patients received two sets of ablations (two ablations at of 300 Watts at $12 \mathrm{~J} / \mathrm{cm}^{2}$ for each set), with vigorous scraping and washes to remove desquamated epithelium between the two sets of treatments. 3D-OCT imaging was performed before, and after each set of ablations. For the CSA treatment, liquid nitrogen was sprayed to freeze the tissue for 20 seconds. The tissue was allowed to thaw completely, followed by another 20 seconds freezing. 3D-OCT imaging was performed before and immediately after the completion of CSA. Imaging was conducted with a spiral-scanning catheter introduced through the biopsy channel of the esophagogastroduodenoscope enabling simultaneous video endoscopy.

A representative of 3D-OCT volumetric dataset of treated site from a 32 year old patient right after RFA treatment is shown in Figure 4.6. These three orthoplanes show 3D-OCT data spanning the RFA treatment site immediately after the first set of ablations, with coloered doted lines indicating the locations of the complementary planes. At a depth of $110 \mathrm{um}$, the en face $\mathrm{XZ}$ orthoplane shows clear delineation between regions with and without hyperscattering features, which are consistent with burned esophageal tissues. The burned tissues were also seen from conventional endoscopy examination as patches of white debris.

As reported in previous study, cross-sectional orthoplanes revealed clear differences in layered architecture in the esophagus [10]. In this study, the cross-sectional imaging capability was also used to characterize the tissue morphology changes after the ablation therapies. Superficial burned tissues show hyperscattering features. Among all the patients receiving RFA therapy, the depth of architectural changes were measured to be $\sim 250$ um (range $200-300$ um).

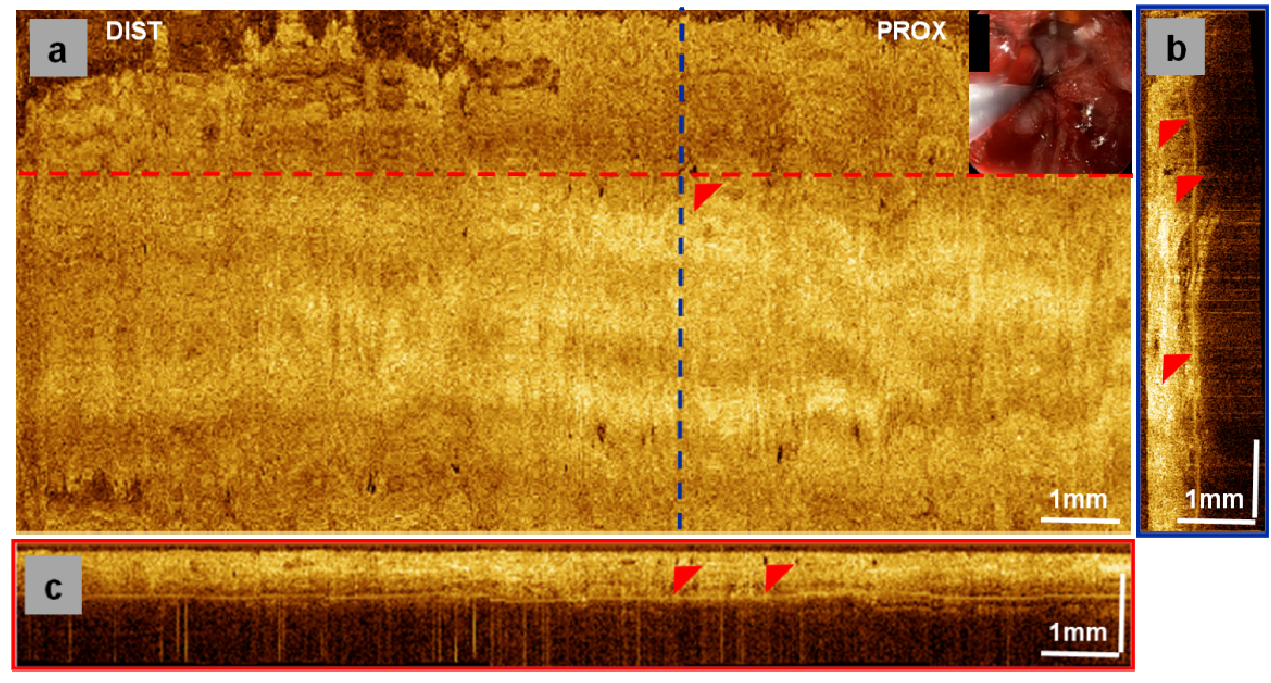

Figure 4.6. Volumetric rendering of $3 \mathrm{D}-\mathrm{OCT}$ data from the $\mathrm{BE}$ region right after the first RFA set. Orthoplanes are shown in purple, blue, and green. Inset shows a video endoscopy image with the 3D-OCT probe in position prior to image acquisition. 
A representative of 3D-OCT volumetric dataset of treated site from a 72 year old patient right after CSA treatment is shown in Figure 4.7. These three orthoplanes show 3D-OCT data spanning the CSA treatment site immediately after the procedure. At a depth of $170 \mathrm{um}$, the en face XZ OCT images illustrated clear delineation between regions with and without edema. Conventional endoscopy shows the distal esophagus after the tissue was thawed completely. Compared to RFA, there was minimal desquamation but edematous tissue changes creating a "spongy" appearance observed as hyposcattering structures reaching a depth of $\sim 650 \mathrm{um}$. These results suggest that 3D-OCT imaging can be a useful tool for providing immediate feedback about therapy induced architectural changes on esophageal tissues.

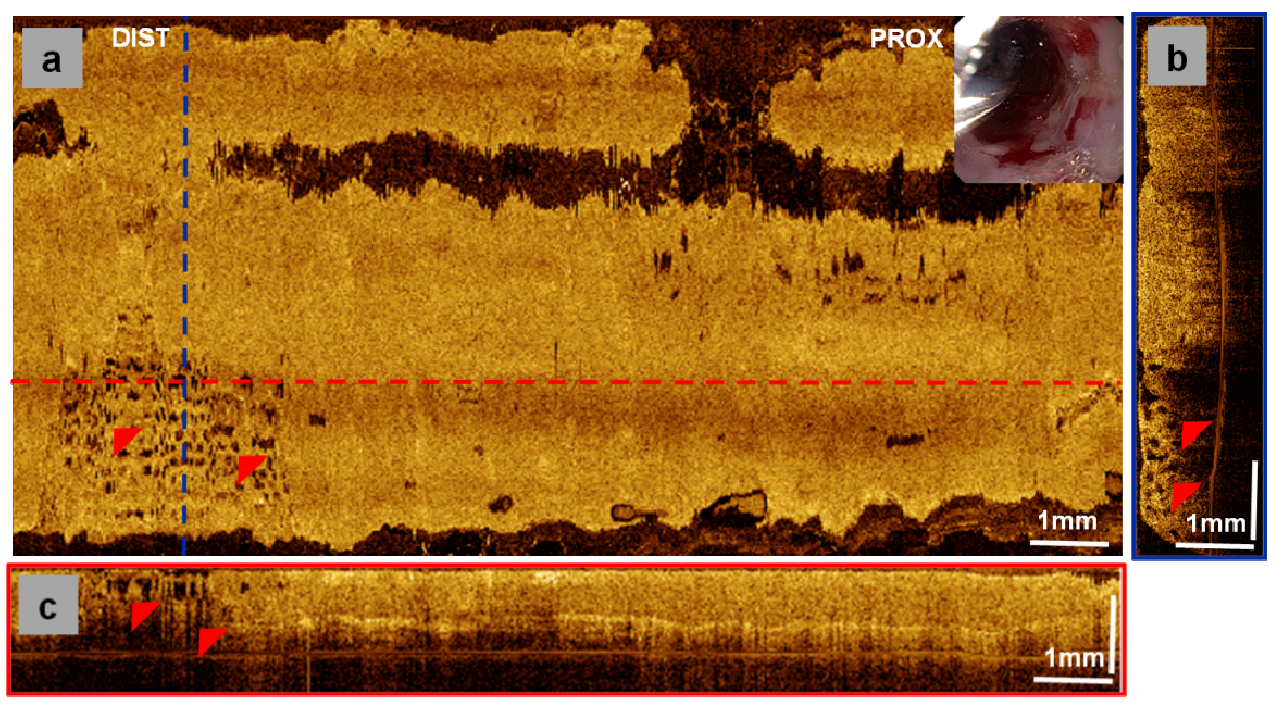

Figure 4.7. Volumetric rendering of $3 \mathrm{D}-\mathrm{OCT}$ data from the $\mathrm{BE}$ region right after the first CSA set. Orthoplanes are shown in purple, blue, and green. Inset shows a video endoscopy image with the 3D-OCT probe in position prior to image acquisition.

The comparison between RFA and CSA is summarized in Table 4.1. In all patients treated with RFA, burned and desquamated tissues appeared hyperscattering, measuring $\sim 250 \mathrm{um}$ in thickness. The second RFA set at the same site caused an additional $\sim 250$ um ablation, giving a combined $\sim 500$ um ablation for each treatment session. In patients in the CSA group, tissue architectural changes with edema and "spongy" appearance caused by freezing were observed up to $\sim 650$ um in depth. Compared to CSA, RFA caused more visible architectural changes observed on 3D-OCT imaging (including sloughing mucosa and more visible debris). However, architectural changes caused by CSA are deeper.

\begin{tabular}{|c|c|c|}
\hline Ablation Method & RFA & CSA \\
\hline \# of ROls & 37 & 7 \\
\hline Characteristics & $\begin{array}{c}\text { Burned tissues with } \\
\text { hyperscattering } \\
\text { desquamation and debris }\end{array}$ & $\begin{array}{c}\text { Edema and "spongy" } \\
\text { appearance }\end{array}$ \\
\hline $\begin{array}{c}\text { Maximum Depth of } \\
\text { Architectural Changes } \\
\text { (mean } \pm \text { SD) }\end{array}$ & $\begin{array}{l}\cdot 251 \text { um } \pm 34 \text { um for each } \\
\text { set of ablations } \\
\text {-502um } \pm \text { 48um for the } \\
\text { whole treatment }\end{array}$ & $\begin{array}{l}\text { 660um } \pm 14 \text { um for the whole } \\
\text { treatment }\end{array}$ \\
\hline
\end{tabular}

Table 4.1. Summary of the comparison between RFA and CSA 
While endoscopic 3D-OCT does not possess the same magnification or contrast as conventional histopathologic analysis, it is capable of visualizing larger and deeper tissue volumes in vivo. RFA produces greater tissue architectural changes and CSA shows changes in deeper tissue structures. The ability to detect tissue structural changes following different ablation therapies makes 3D-OCT an ideal tool to assess the treated regions, which might help identifying areas for retreatment and guide refinements in treatment dosing.

\section{References}

[1] "Cancer Facts and Figures," American Cancer Society 20102010.

[2] D. K. Rex, "Maximizing detection of adenomas and cancers during colonoscopy," American Journal of Gastroenterology, vol. 101, pp. 2866-2877, 2006.

[3] S. J. Spechler, "Dysplasia in Barrett's esophagus: limitations of current management strategies," Am J Gastroenterol, vol. 100, pp. 927-35, 2005.

[4] G. J. Tearney, M. E. Brezinski, J. F. Southern, B. E. Bouma, S. A. Boppart, and J. G. Fujimoto, "Optical biopsy in human gastrointestinal tissue using optical coherence tomography," The American journal of gastroenterology, vol. 92, pp. 1800-4, 1997.

[5] M. A. Choma, M. V. Sarunic, C. H. Yang, and J. A. Izatt, "Sensitivity advantage of swept source and Fourier domain optical coherence tomography," Optics Express, vol. 11, pp. 2183-2189, 2003.

[6] J. F. de Boer, B. Cense, B. H. Park, M. C. Pierce, G. J. Tearney, and B. E. Bouma, "Improved signal-to-noise ratio in spectral-domain compared with time-domain optical coherence tomography," Optics Letters, vol. 28, pp. 2067-2069, 2003.

[7] R. Leitgeb, C. K. Hitzenberger, and A. F. Fercher, "Performance of Fourier domain vs. time domain optical coherence tomography," Optics Express, vol. 11, pp. 889-894, 2003/04/21 2003.

[8] R. Huber, D. C. Adler, and J. G. Fujimoto, "Buffered Fourier domain mode locking: unidirectional swept laser sources for optical coherence tomography imaging at 370,000 lines/s," Optics Letters, vol. 31, pp. 2975-2977, 2006.

[9] D. C. Adler, Y. Chen, R. Huber, J. Schmitt, J. Connolly, and J. G. Fujimoto, "Three-dimensional endomicroscopy using optical coherence tomography," Nature Photonics, vol. 1, pp. 709-716, 2007.

[10] D. C. Adler, C. Zhou, T. H. Tsai, H. C. Lee, L. Becker, J. M. Schmitt, Q. Huang, J. G. Fujimoto, and $\mathrm{H}$. Mashimo, "Three-dimensional optical coherence tomography of Barrett's esophagus and buried glands beneath neosquamous epithelium following radiofrequency ablation," Endoscopy, vol. 41, pp. 773-776, 2009.

[11] D. C. Adler, C. Zhou, T. H. Tsai, J. Schmitt, Q. Huang, H. Mashimo, and J. G. Fujimoto, "Threedimensional endomicroscopy of the human colon using optical coherence tomography," Optics Express, vol. 17, pp. 784-796, 2009.

[12] S. Zhou, Y. Li, A. T. H. Lu, P. F. Liu, M. L. Tang, S. C. Yiu, and D. Huang, "Reproducibility of Tear Meniscus Measurement by Fourier-Domain Optical Coherence Tomography: A Pilot Study," Ophthalmic Surgery Lasers \& Imaging, vol. 40, pp. 442-447, 2009.

[13] B. E. Bouma, G. J. Tearney, C. C. Compton, and N. S. Nishioka, "High-resolution imaging of the human esophagus and stomach in vivo using optical coherence tomography," Gastrointestinal endoscopy, vol. 51(4) Pt 1, pp. 467-74, 2000.

[14] S. Jäckle, N. Gladkova, F. Feldchtein, A. Terentieva, B. Brand, G. Gelikonov, V. Gelikonov, A. Sergeev, A. Fritscher-Ravens, J. Freund, U. Seitz, S. Schröder, and N. Soehendra, "In vivo endoscopic optical coherence tomography of esophagitis, Barrett's esophagus, and adenocarcinoma of the esophagus," Endoscopy, vol. 32, pp. 750-5, 2000.

[15] M. V. Sivak, Jr., K. Kobayashi, J. A. Izatt, A. M. Rollins, R. Ung-Runyawee, A. Chak, R. C. Wong, G. A. Isenberg, and J. Willis, "High-resolution endoscopic imaging of the GI tract using optical coherence tomography," Gastrointestinal endoscopy, vol. 51(4) Pt 1, pp. 474-9, 2000.

[16] X. D. Li, S. A. Boppart, J. Van Dam, H. Mashimo, M. Mutinga, W. Drexler, M. Klein, C. Pitris, M. L. Krinsky, M. E. Brezinski, and J. G. Fujimoto, "Optical coherence tomography: advanced technology for the endoscopic imaging of Barrett's esophagus," Endoscopy, vol. 32, pp. 921-30, 2000. 
[17] J. M. Poneros, S. Brand, B. E. Bouma, G. J. Tearney, C. C. Compton, and N. S. Nishioka, "Diagnosis of specialized intestinal metaplasia by optical coherence tomography," Gastroenterology, vol. 120, pp. 7-12, 2001.

[18] G. Isenberg, M. V. Sivak, A. Chak, R. C. K. Wong, J. E. Willis, B. Wolf, D. Y. Rowland, A. Das, and A. Rollins, "Accuracy of endoscopic optical coherence tomography in the detection of dysplasia in Barrett's esophagus: a prospective, double-blinded study," Gastrointestinal Endoscopy, vol. 62, pp. 825-831, 2005.

[19] J. A. Evans, J. M. Poneros, B. E. Bouma, J. Bressner, E. F. Halpern, M. Shishkov, G. Y. Lauwers, M. Mino-Kenudson, N. S. Nishioka, and G. J. Tearney, "Optical coherence tomography to identify intramucosal carcinoma and high-grade dysplasia in Barrett's esophagus," Clinical Gastroenterology and Hepatology, vol. 4, pp. 38-43, 2006.

[20] X. Qi, M. V. Sivak, G. Isenberg, J. E. Willis, and A. M. Rollins, "Computer-aided diagnosis of dysplasia in Barrett's esophagus using endoscopic optical coherence tomography," Journal of Biomedical Optics, vol. 11, p. 10, 2006.

[21] Y. Chen, A. D. Aguirre, P. L. Hsiung, S. Desai, P. R. Herz, M. Pedrosa, Q. Huang, M. Figueiredo, S. W. Huang, A. Koski, J. M. Schmitt, J. G. Fujimoto, and H. Mashimo, "Ultrahigh resolution optical coherence tomography of Barrett's esophagus: preliminary descriptive clinical study correlating images with histology," Endoscopy, vol. 39, pp. 599-605, 2007.

[22] R. D. Odze and G. Y. Lauwers, "Histopathology of Barrett? esophagus after ablation and endoscopic mucosal resection therapy," Endoscopy, vol. 40, pp. 1008-1015, 2008.

[23] V. K. Sharma, H. J. Kim, A. Das, P. Dean, G. DePetris, and D. E. Fleischer, "A prospective pilot trial of ablation of Barrett? esophagus with low-grade dysplasia using stepwise circumferential and focal ablation (HALO system)," Endoscopy, vol. 40, pp. 380-387, 2008.

[24] M. H. Johnston, "Cryotherapy and other newer techniques," Gastrointest Endosc Clin N Am, vol. 13, pp. 491-504, 2003.

[25] M. H. Johnston, J. A. Eastone, J. D. Horwhat, J. Cartledge, J. S. Mathews, and J. R. Foggy, "Cryoablation of Barrett's esophagus: a pilot study," Gastrointestinal Endoscopy, vol. 62, pp. 842848, 2005.

[26] H. Barr, N. Krasner, P. B. Boulos, P. Chatlani, and S. G. Bown, "PHOTODYNAMIC THERAPY FOR COLORECTAL-CANCER - A QUANTITATIVE PILOT-STUDY," British Journal of Surgery, vol. 77, pp. 93-96, 1990.

[27] H. Barr, N. Stone, and B. Rembacken, "Endoscopic therapy for Barrett's oesophagus," Gut, vol. 54, pp. 875-884, 2005.

[28] D. Huang, E. A. Swanson, C. P. Lin, J. S. Schuman, W. G. Stinson, W. Chang, M. R. Hee, T. Flotte, K. Gregory, C. A. Puliafito, and J. G. Fujimoto, "Optical Coherence Tomography," Science, vol. 254, pp. 1178-1181, 1991.

[29] C. Zhou, D. C. Adler, L. Becker, Y. Chen, T.-H. Tsai, M. Figueiredo, J. M. Schmitt, J. G. Fujimoto, and $\mathrm{H}$. Mashimo, "Effective treatment of chronic radiation proctitis using radiofrequency ablation " Therapeutic Advances in Gastroenterology, vol. 2, pp. 149-156, 2009. 


\section{Optical Coherence Tomography and Microscopy for Pathology Laboratory Imaging}

\section{Sponsors}

National Institutes of Health - R01-CA75289-13, R01-NS057476-02, R01HL095717-01

National Science Foundation - BES-0522845

Air Force Office of Scientific Research - FA9550-07-1-0101, FA9550-07-1-0014

\section{Project Staff}

Prof. James G. Fujimoto Dr. Chao Zhou, Dr. Aaron D. Aguirre, M.D., Ph.D., Hsiang-Chieh Lee, Tsung-Han Tsai (MIT)

Dr. David W. Cohen, M.D., Dr. Amy Mondelblatt, M.D., Dr. Yihong Wang, M.D., Dr. James L. Connolly, M.D. (BIDMC)

Excisional biopsy is the current gold standard for disease diagnosis; however, it requires a relatively long processing time and it may also suffer from unacceptable rates due to sampling errors. OCT can provide two- and three-dimensional structural information of the tissue specimen [1]. Utilizing broadband light source, ultrahigh resolution OCT enables imaging of the specimens with axial resolution of 1-2 um in tissue [2, 3]. However, in order to generate cross-sectional OCT images with sufficient depth of field, the transverse resolution of OCT is limited due to the lower numerical aperture (NA) focusing that is required. Generally, the lower NA focusing ustilized for OCT is insufficient to resolve cellular features in human tissue specimens. In order to improve the imaging performance of OCT to higher transverse resolution, our group is developing optical coherence microscopy (OCM), which combines OCT coherent detection with conventional confocal microscopy. Figure 5.1 compares the imaging performance of various modalities including ultrasound, OCT, OCM, and standard confocal microscopy. OCM enables imaging with enhanced penetration depth compared to standard confocal microscopy, while improving the transverse image resolution compared to OCT.

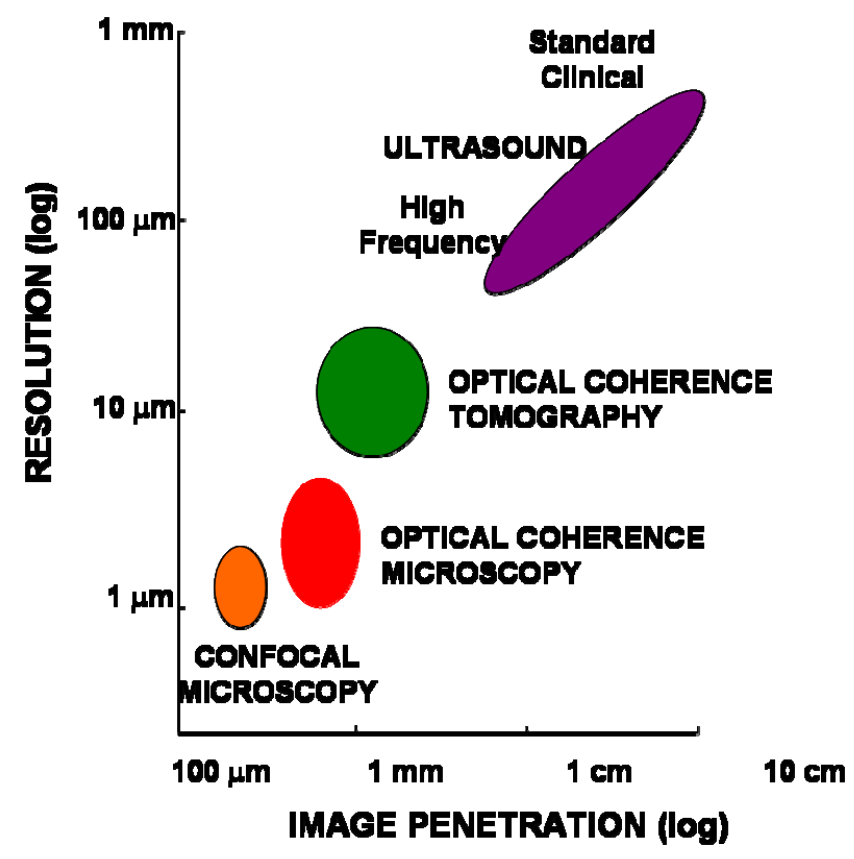

Figure 5.1. Transverse image resolution and image penetration in optical coherence microscopy (OCM). OCM can enhance image penetration when compared to confocal microscopy alone, while significantly improving transverse resolution in OCT to enable cellular-level imaging. 
OCM overcomes the depth-of-field limitation in conventional OCT by imaging the specimen in the en face plane rather than the cross-sectional plane. En face imaging requires the optical path length of the reference arm to be exactly matched to the sample arm focus, while raster scanning the focused spot over the specimen. En face imaging not only eliminates the need of path length scanning in the axial direction, but also allows the use of tight focusing optics to improve transverse resolution. However, due to the high NA objective used, the field of view of OCM is smaller than that of OCT. OCT provides architectural features over a filed of 3-6 mm field, while OCM enables cellular level imaging but with reduced field of view of $100-500$ um field.

OCM combines features of two distinct optical sectioning techniques - confocal gating and coherence gating. The overall sectioning power is determined by the multiplicative effect of both the coherence gate width and confocal gate width. The coherence gate width is determined by the bandwidth of the light source, while the degree of confocal rejection of unfocused scattered light can be varied by changing the NA of the focusing optics. Our group and others have demonstrated that OCM can provide images with improved imaging depth when compared to standard confocal microscopy alone [4-6]. The implementation of coherence gating enables high-sensitivity, shot-noise limited detection while reducing scattered light and increases the imaging depth by a factor of 2-3 over standard confocal microscopy. Figure 5.2 compares the axial resolution and transverse resolution as a function of the NA of the focusing optics to demonstrate the operating limit of OCM. The axial resolution degrades more quickly with decreasing NA of the objective. However, there exist regions where the NA is sufficient to resolve cellular features in the transverse direction but not in the axial direction. The addition of a short coherence gate helps improving the axial resolution and thus enables the cellular-level imaging with lower NA objectives. The implementation of lower NA focusing optics can enable cellular imaging with small diameter probes that are compatible with standard endoscopic or laparoscopic procedures.

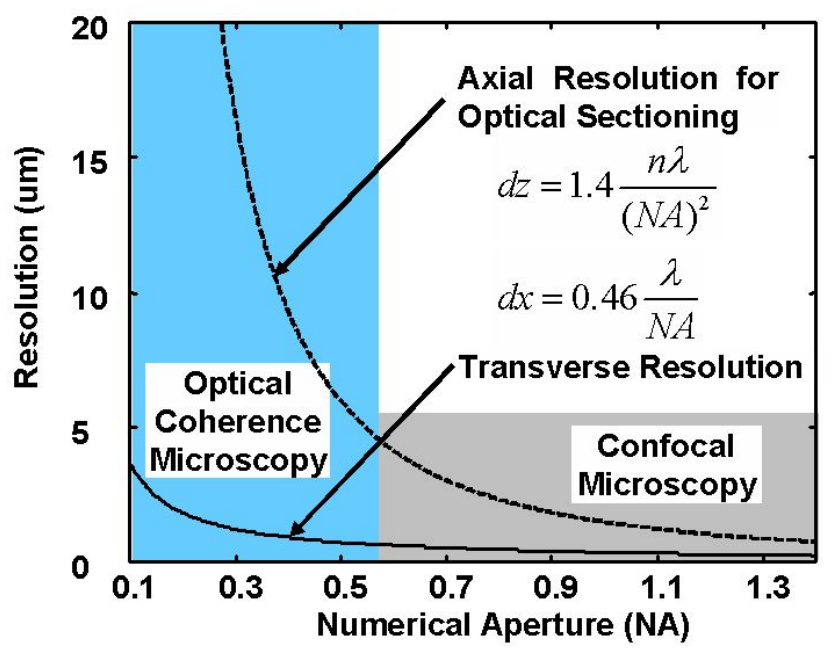

Figure 5.2. Numerical aperture requirement for OCM compared to confocal microscopy. OCM can image with a high transverse resolution at a much lower numerical aperture than confocal microscopy because it does not depend on high axial resolution for optical sectioning.

Ex vivo imaging in the pathologic laboratory provides access to a wide range of pathological specimens which would not be available outside the clinic. It also enables a more controlled comparison to histology in order to validate new imaging modalities. The integrated 3D-OCT and OCM system enables multi-scale imaging capability. Integrated OCT/OCM can provide OCT architectural information from the specimen over a large field of view, while OCM can achieve cellular resolution, but with a reduced field of view.

Our group has an ongoing collaboration with Dr. James Connolly, MD at the Beth Israel Deaconess Medical Center and Harvard Medical School to investigate OCT and OCM for imaging pathology. A wide range of human pathologic specimens, including colon, thyroid, breast, kidney, lymph nodes, female 
reproductive tract, and others are being investigated. Studies are performed on freshly excised pathologic specimens in the pathologic laboratory. A more detail description of the integrated OCT/OCM system can be found in the reference [7]. The OCT/OCM images are generated using a compact femtosecond $\mathrm{Nd}$ :Glass laser, spectrally broadened utilizing supercontinuum generation technique to provide an output spectrum >200 nm in bandwidth centered at $1060 \mathrm{~nm}[8,9]$. This light source enables OCT/OCM images with an axial resolution <4 um. A fiber-optic scanning confocal microscope is implemented in the sample arm shared by both the OCT and OCM system. Objectives with different magnifications were mounted on a turret, which allows rapid switching between OCT and OCM imaging modes. The transverse resolution of the OCT and en face OCM images are $14 \mathrm{um}$ and $2 \mathrm{um}$, respectively. Each cross-sectional OCT image $(1.5 \mathrm{~mm} \times 3 \mathrm{~mm}, \mathrm{Z} \times \mathrm{X})$ is acquired at 1 frame/second. OCM images covering $400 \times 400 \mathrm{um}$ are generated at 2 frames/second. 3D OCM datasets can be generated by translating the sample stage in the $z$ direction.

\subsection{Integrated OCT and OCM Imaging of Human Thyroid Pathology}

Thyroid cancer is the most common malignancy of the endocrine system [10]. Approximately 37,200 new cases and 1,630 thyroid cancer deaths are expected in the United States in 2010 [11]. Various methods are used for the detection of and screening of thyroid nodules for malignancy. These include clinical examination, various imaging methods, and ultrasound guided fine needle aspiration (FNA). Thyroid cancer commonly presents as a cold (inactive) nodule on radio-isotope scanning. Up to $40 \%$ of adults have a thyroid nodule detected by either palpation or ultrasound [12-15]. Imaging techniques that can aid in the differentiation between benign and malignant thyroid nodules, which may require surgery, are of great interest.

In the present study [16], we employ an integrated OCT and OCM system to assess benign and malignant thyroid tissue ex vivo based on intrinsic optical contrast in freshly excised human thyroid specimens. Images of normal and pathologic tissue were compared with histological sections in order to recognize which histomorphologic features could be visualized using integrated OCT and OCM imaging. The results provide a basis for interpretation of future OCT and OCM images of the thyroid tissues and suggest the possibility of future in vivo evaluation of thyroid pathology.

As shown in Figure 5.3, surfaces of 640 2D-OCT cross-sectional images were detected and flattened in post-processing to allow en face image planes to be viewed at constant depth. En face slices of OCT images $(3 \times 1.5 \mathrm{~mm})$ were reconstructed from the $3 \mathrm{D}$ data sets by averaging over 10 um intervals in the axial direction to reduce speckle noise.

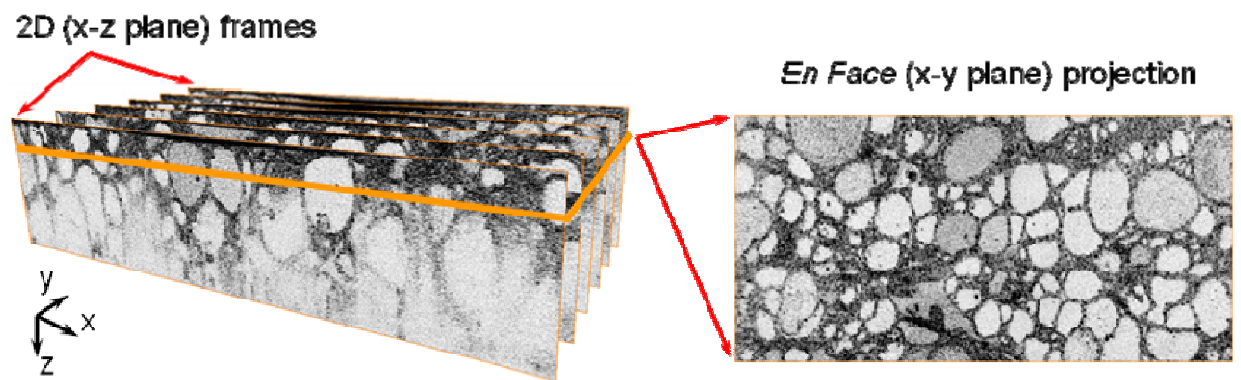

Figure 5.3. En face OCT image (right) was constructed from the $3 \mathrm{D}$ volumetric data set (left), consisting 640 2D cross-sectional OCT images.

Figure 5.4 shows an example of Hashimoto's thyroiditis, which is an autoimmune disease characterized by extensive lymphocytic infiltration with germinal center formation and Hürthle cell change. In addition to the observation of enlarged and distorted follicles, from the en face OCT and OCM images, we observe marked increased interfollicular cellular density with nodule configuration and central palor, which corresponds well with interstitial lymphocytic infiltrate and germinal center formation. The corresponding histology (Figs. 5.4C and D) matches well with the OCT and OCM images. 
Figure $\mathbf{5 . 5}$ shows an example of the follicular variant of papillary carcinoma. The nodular thyroid shows a homogenous micro-follicullar pattern (Figs. 5.5A and C). Details of the micro-follicles can be seen in the OCM image. The size of the micro-follicles is $\sim 50$ um, consistent with histology. Figure $\mathbf{5 . 6}$ shows en face OCM images from various depths. Micro-follicles can be clearly identified about 180 um below the tissue surface.

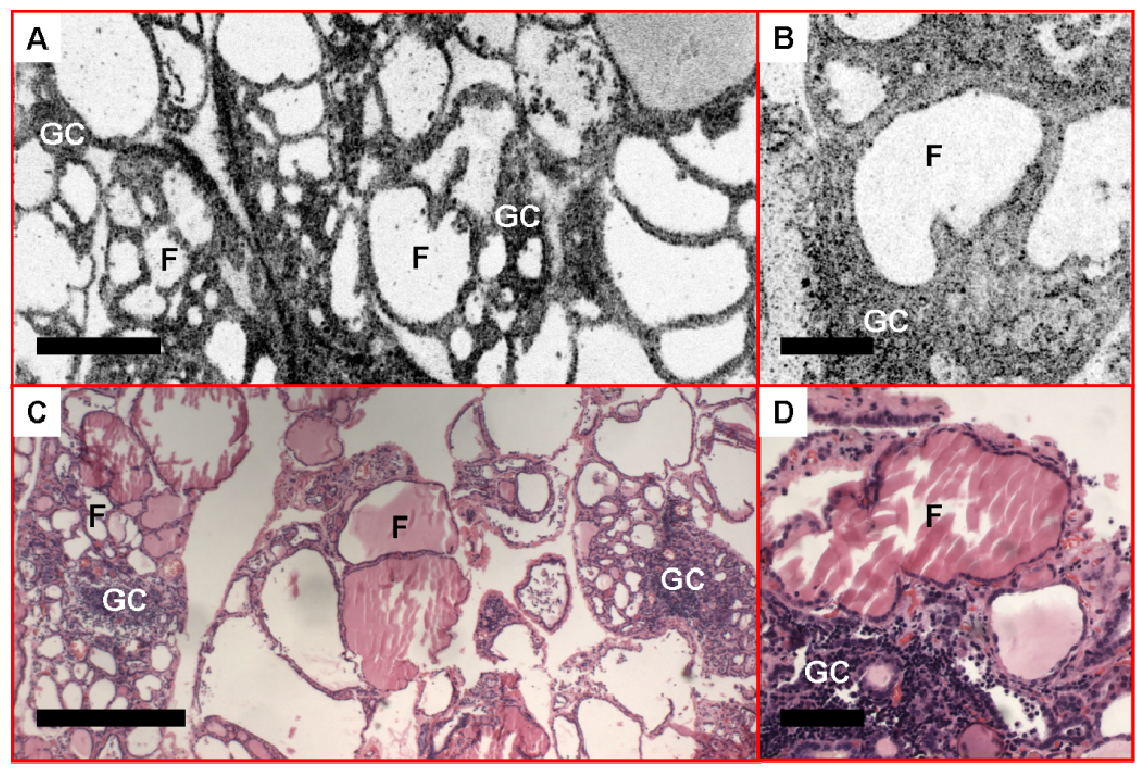

Figure 5.4. Example of Hashimoto's thyroiditis. En face OCT (A) and OCM (B), obtained about 230 um and 50 um below the tissue surface respectively, demonstrate enlarged and distorted follicles $(F)$, increased interfollicular cellular density, interstitial lymphocytic infiltration and germinal center (GC) formation. (C) and (D), corresponding H\&E slides (4x and 20x respectively). Scale bars, 500 um in $(A, C)$ and $100 \mathrm{um}$ in $(B, D)$.

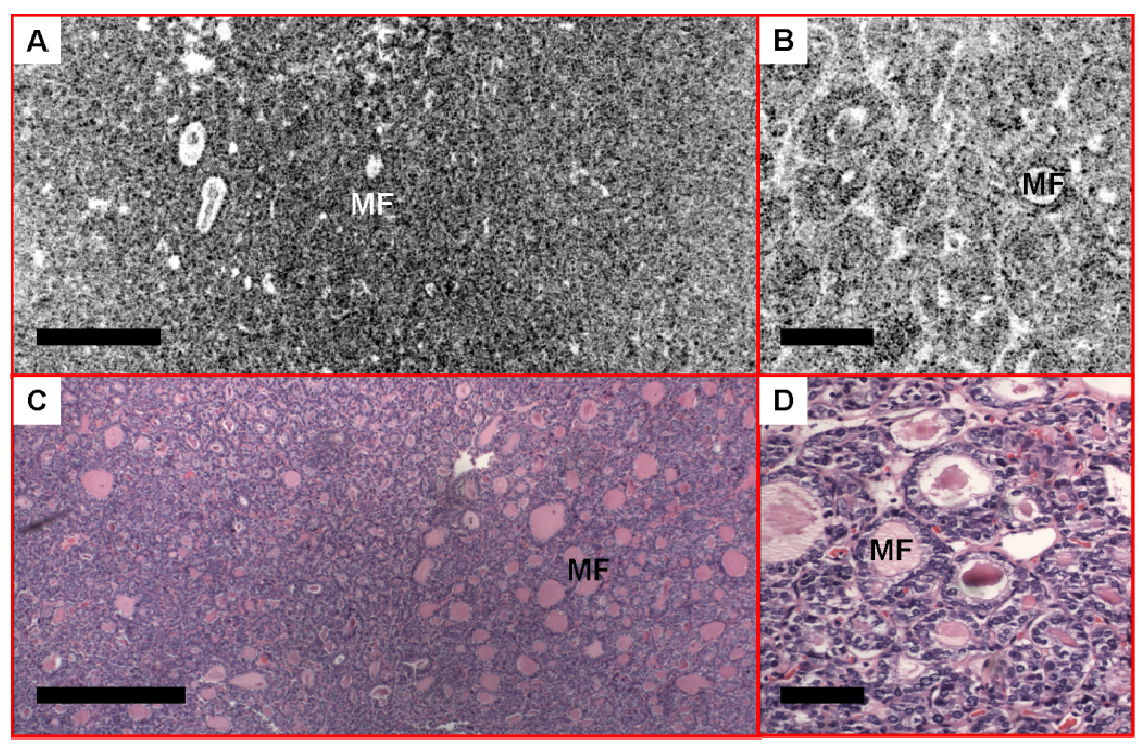

Figure 5.5. A representative case of the follicular variant of papillary carcinoma. En face OCT (A) showed a homogeneous micro-follicular (MF) pattern, where the details can be seen under OCM (B). The en face OCT and OCM images were obtained about 200 um and 50 um below the tissue surface respectively. The size of the micro-follicles is approximately $50 \mathrm{um}$, consistent with the H\&E histology in (C, D, 4x and 20x respectively). Scale bars, 500 um in (A, C) and 100 um in (B, D). 


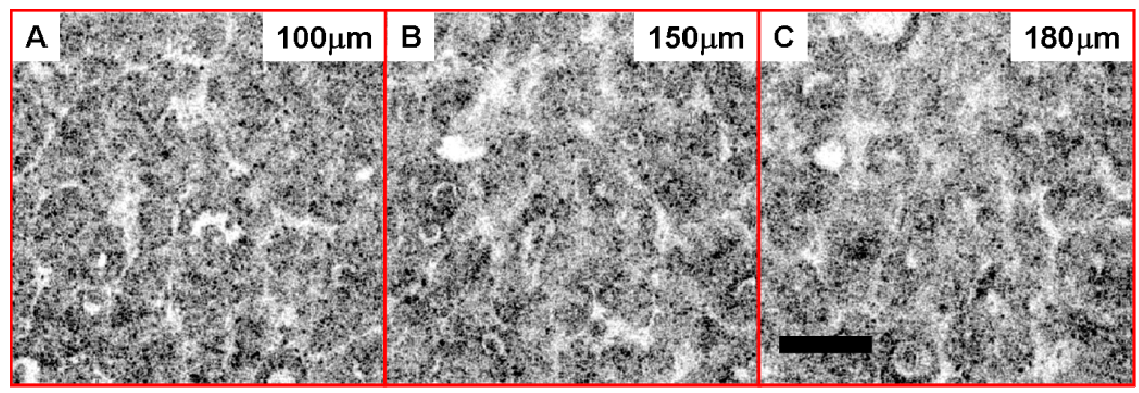

Figure 5.6. En face OCM images of follicular variant of papillary carcinoma from various depths. The papillae structure can be visible from 180um below the tissue surface. Scale bar, 100um.

The OCT and OCM technologies presented in the current study have the potential to be a useful complementary technique for the evaluation of thyroid nodules. The axial and transverse resolutions of OCT and OCM are 1-2 orders of magnitude finer than the state-of-the-art ultrasound technology. The integrated OCT and OCM system allows seamlessly switching between low and high magnifications, in a way similar to traditional microscopy. The ability to visualize tissue morphology at multiple scales is very important for pathologists to differentiate clinical relevant features. As a result, characteristic architectural and cellular features from normal thyroid and benign and malignant thyroid diseases were successfully visualized at multiple resolution scales in excised specimens, without exogenous contrast agents or histological processing.

\subsection{Integrated OCT and OCM Imaging of Human Breast Pathology}

Excluding skin cancers, breast cancer has the highest incidence in women, with an estimated 207,090 new cases of invasive breast carcinoma and 54,010 new cases of in situ carcinoma expected to occur in the United States (U.S.) in 2010 [17]. Even though breast cancer is the second leading cause of cancer death in women, with an estimated 39,840 occurring in 2010 , breast cancer mortality has declined in the last decade. This decrease in mortality is largely attributed to the increased use of screening mammography and improved effectiveness of treatments [17]. Image-guided core biopsy and surgical excisional biopsy are most often used in the diagnostic workup of breast lesions. Compared to open surgical biopsy, core biopsy is less invasive and is therefore considered a gold standard for the diagnosis of breast cancer [18]. However, core biopsy can have a disadvantage of false-negative rates that result from limitations in tissue sampling [19-22]. The false-negative rate for palpation-guided and stereotacticguided core biopsy ranges from $0-13 \%[21,23]$ and $0.2 \%-8.9 \%[21,24]$, respectively. Ultrasound-guided core biopsy has been shown to reduce the false negative rate to $0 \%-3.6 \%[21,22,25,26]$. Imaging techniques providing more accurate sampling capabilities in real-time can improve the diagnostic performance of core biopsies. The emergence of breast conserving surgeries, such as partial mastectomy, allows preservation of the breast without compromising survival. The surgical margin status is considered a strong predictor for local recurrence following partial mastectomy [27-29]. In clinical practice, the combination of intra-operative gross examination and post-operative histologic examination are employed to evaluate the surgical margins. However, as many as $\sim 40 \%$ of patients require a second surgical procedure due to positive or close margins [30,31]. In addition, evaluation of sentinel and axillary nodal involvement is critical for staging breast cancers [32,33]. Post-operative histologic examination remains the gold standard in assessing the status of lymph nodes. However, sentinel and axillary lymph nodes resections may lead to potential complications such as lymphedema [34]. Frozen section analysis has been used to evaluate surgical margins and nodes status intra-operatively [35, 36]. However, the analysis is time-consuming and the sampling area is limited. Therefore, a real-time, non-destructive imaging method that has a large sampling field, micron-scale resolution and depth-resolved imaging capability are desirable for intra-operative evaluation of breast cancer. 
OCT and OCM are promising techniques for real-time, high resolution imaging of tissue morphology and have the potential to be used for these important clinical applications. In the current study, we employed an integrated OCT and OCM system developed in our laboratory to study freshly excised human breast tissue with various pathologic diagnoses ex vivo based on intrinsic optical contrast. Few studies using integrated OCT and OCM imaging have been performed to date [37, 38], largely due to the lack of advanced OCM instrumentation. The objective of our study was to establish imaging features observed in OCT and OCM and compare these with histology to identify characteristic features of breast lesions that can be visualized with OCT and OCM. The results provide a basis for the interpretation of future OCT and OCM breast tissue images and lay the foundation for future in vivo optical evaluation of breast lesions.

Forty-four breast specimens were imaged from 22 patients (median age, 51 years; range 20-90). Thirtyfour benign breast specimens were imaged. The specimens with benign diagnosis include fibroadenoma $(n=4)$, benign fibrocystic disease $(n=13)$, fat necrosis $(n=3)$, usual ductal hyperplasia $(U D H, n=4)$ and normal breast parenchyma $(n=14)$. Ten specimens were diagnosed as breast carcinoma including invasive ductal carcinoma $(n=5)$, invasive lobular carcinoma $(n=4)$, mucinous (colloid) carcinoma $(n=$ 1 ), ductal carcinoma in situ (DCIS, $n=5$ ) and lobular carcinoma in situ (LCIS, $n=2$ ). Specimens were classified based upon histologic diagnosis by experienced pathologists.

Figure 5.7 shows characteristic features observed in normal breast tissue. Adipose tissue is apparent on the OCT image as round, hyposcattering (white) circular structures. Blood vessels are identified as long, hyposcattering tubes with hyperscattering (black) walls. Loose fibrous stroma appears as angulated black-and-white short linear reticulations with high contrast. Normal breast terminal duct lobular units (TDLUs) appear as pale regions that are relatively homogeneous in the en face OCT image. Under high magnification with OCM, individual TDLUs can be identified, with hyposcattering lumina clearly seen (Fig. 5.7B, arrows).

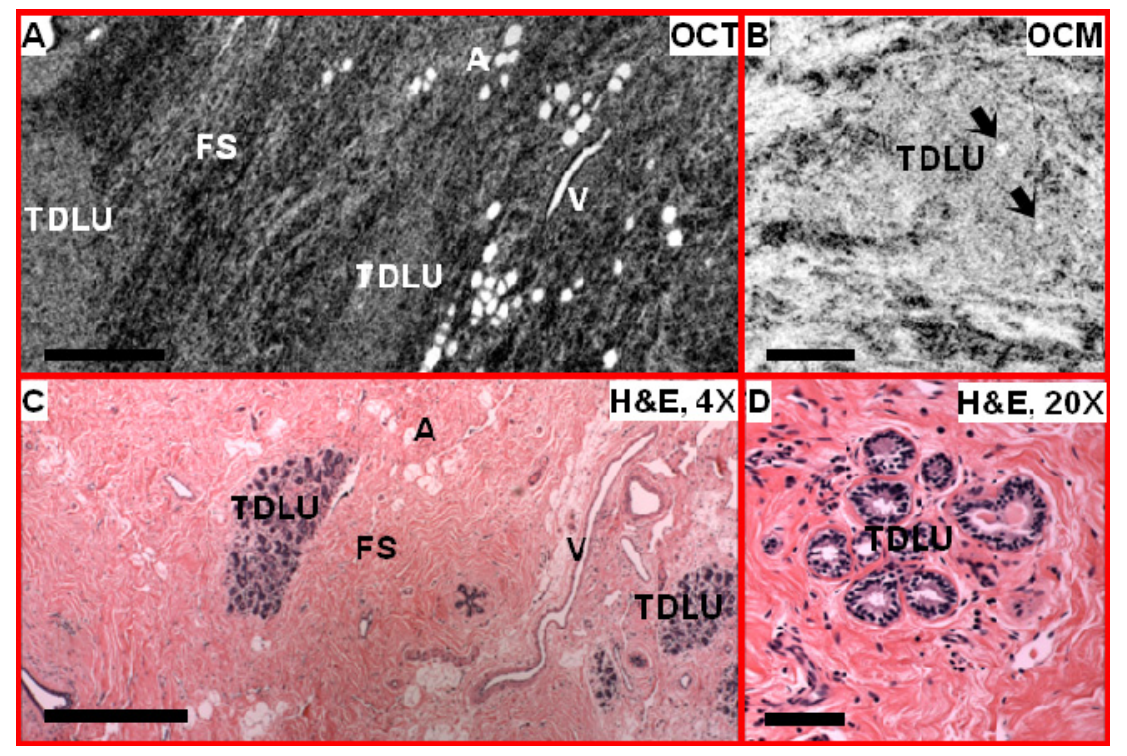

Figure 5.7. (A) En face OCT projection of normal breast tissue shows fiber stroma "FS", blood vessels "V", adipose tissue "A" and terminal duct lobular units "TDLU". (B) OCM reveals details of TDLU. Glandular lumina can be clearly seen as hyposcattering circles (arrow). (C, D) Corresponding histology. Scale bars, 500um in (A, C) and 100um in (B, D).

Figure 5.8 shows several representative features observed in benign breast lesions. Fig. $\mathbf{5 . 8 A}$ is an example of a fibroadenoma, in which breast ducts are compressed by surrounding stroma. The OCT image demonstrates the well-circumscribed nodule on low magnification; the interface consists of a smooth border separating hyperscattering dense fibrous tissue. Fig. 5.8B shows characteristic features of fat necrosis, in which necrotic (hyposcattering) adipocytes of different sizes can be clearly identified in the 
OCT image.

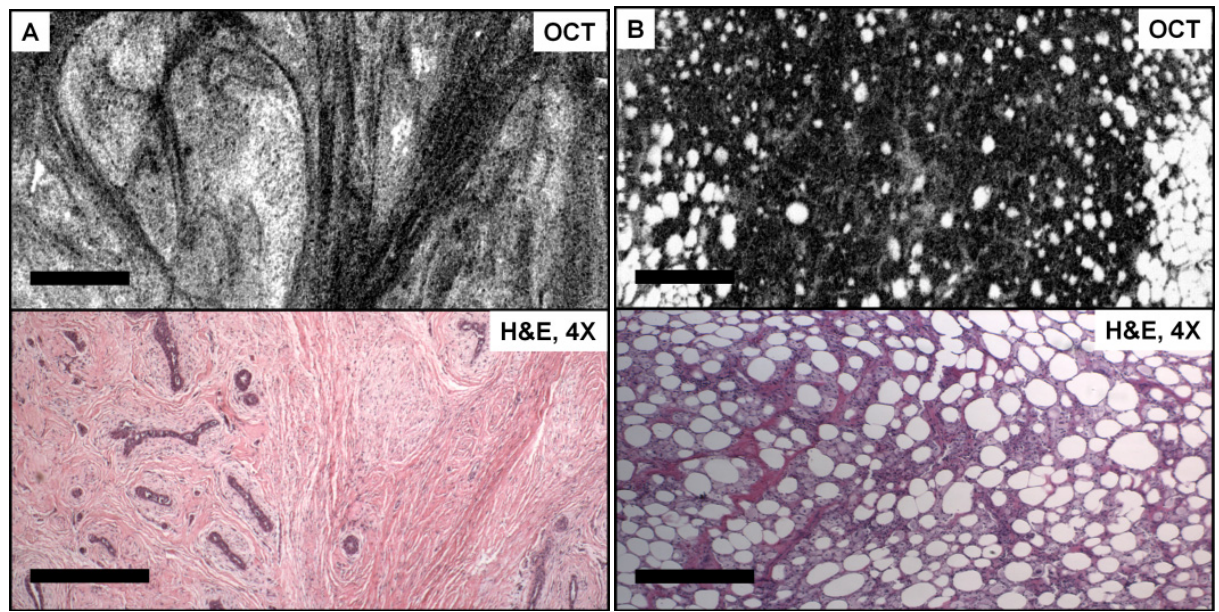

Figure 5.8. Benign breast lesions. (A) OCT and corresponding H\&E histologic section of a fibroadenoma; (B) OCT and corresponding H\&E histologic section of fat necrosis. Scale bars, 500 um.

Figure 5.9 shows an invasive ductal carcinoma with co-existing ductal carcinoma in situ (DCIS, arrows) observed in the en face OCT image. The DCIS regions are clearly delineated by a basement membrane within a background of hyperscattering stromal tissue. In addition, the OCM image in Fig. 5.9B demonstrates small clusters of infiltrating carcinoma. The corresponding histological slides (Figs. 5.9C and D) further confirm these findings. More detailed investigations are needed to establish diagnostic features in OCT and OCM when compared to histology, as well as to assess sensitivity and specificity in a blinded fashion. However, these results demonstrate that OCT and OCM can provide real-time imaging of tissue morphology at multiple resolution scales, and is a promising tool for detecting neoplastic changes.

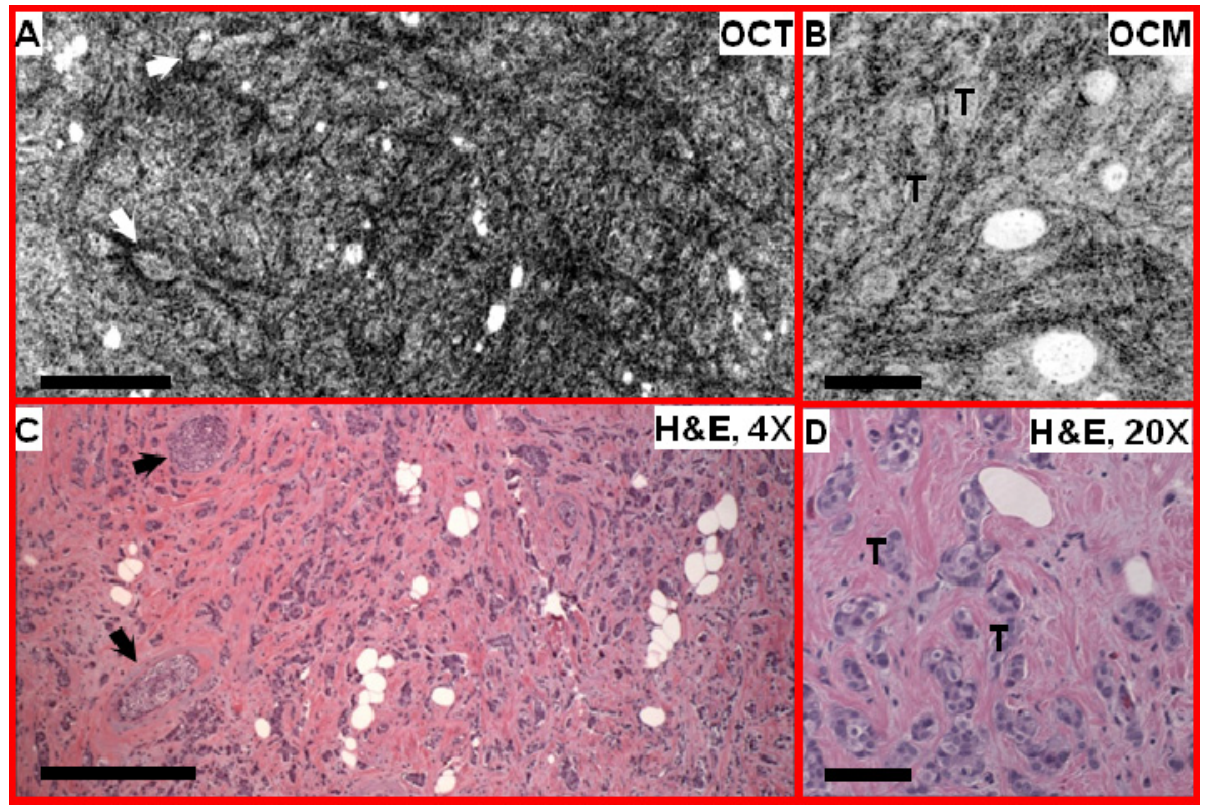

Figure 5.9. Invasive and in situ ductal carcinoma (DCIS) of the breast. Arrows in (A) point to the DCIS involvements in invasive carcinoma. En face OCM image in (B) shows clusters of invasive tumor cells "T". (C, D) Corresponding histology. Scale bars, 500 um in (A, C) and 100 um in (B, D). 


\subsection{Integrated OCT and OCM imaging of human kidney pathology}

Renal cell carcinoma (RCC), the most common type of kidney cancer accounts for approximately $85 \%$ of renal parenchyma malignancies. RCC ranks as one of the common malignancy among adults. There are approximately over 50,000 new cases of kidney cancer diagnosed and 13,000 people died each year [11]. Renal tumors usually start to develop asymptomatically as small masses, and become larger along with the progression. Currently, in most institutions, computed tomography (CT) is the major imaging technique used to evaluate the renal tumors. In addition to CT, magnetic resonance imaging (MRI) and ultrasound (sonography) are complimentary imaging tools for candidates not appropriate for CT scanning. The prognosis of RCC is strongly influenced by the stage of diagnosis. The overall five-year survival rate is larger than $50 \%$ for localized renal tumor while $10 \%$ for those with distant metastasis.

Our group and collaborators explored the feasibility of OCT and OCM imaging in the assessment of renal tumor mass. The integrated OCT/OCM system was deployed to image freshly excised both normal and malignant human kidney tissues. The OCT/OCM image features were correlated with different pathologies. The feasibility of using OCT/OCM images to guided fine needle aspiration (FNA) or core need biopsy procedures to minimize sampling errors was investigated in the study.

Figrue $\mathbf{5 . 1 0}$ shows representative images of normal human kidney using the integrated OCT/OCM system. Round spherical glomeruli as well as the surrounding convoluted tubules are observed in the en face OCT image as shown in Fig. 5.10A, consistent with normal renal cortex. The average size of the glomerulus identified is around 200 um in diameter, while the surrounding tubules measure $\sim 30 \mathrm{um}$. The branching vascular network within the glomerulus is demonstrated as round pale regions surrounded by a hyposcattering ring, which is the Bowman's space. In addition to en face OCT images, cross-sectional OCT image reveals the depth-resolved morphological information of the specimen. Fig. 5.10B shows a cross-section OCT image of the normal kidney cortex, corresponding to the scan position marked in the en face OCT image (Fig. 5.10A). Glomeruli $(G)$ as well as the convoluted tubule $(T)$ are observed from cross-sectional OCT image. Fig. 5.10C shows an OCM image of a glomerulus paired with the corresponding histologic section. Under high magnification, the glomerulus structure can be observed in more detail. The patterns of the glomerulus structures have been shown to correlate with various glomerular diseases. [39].
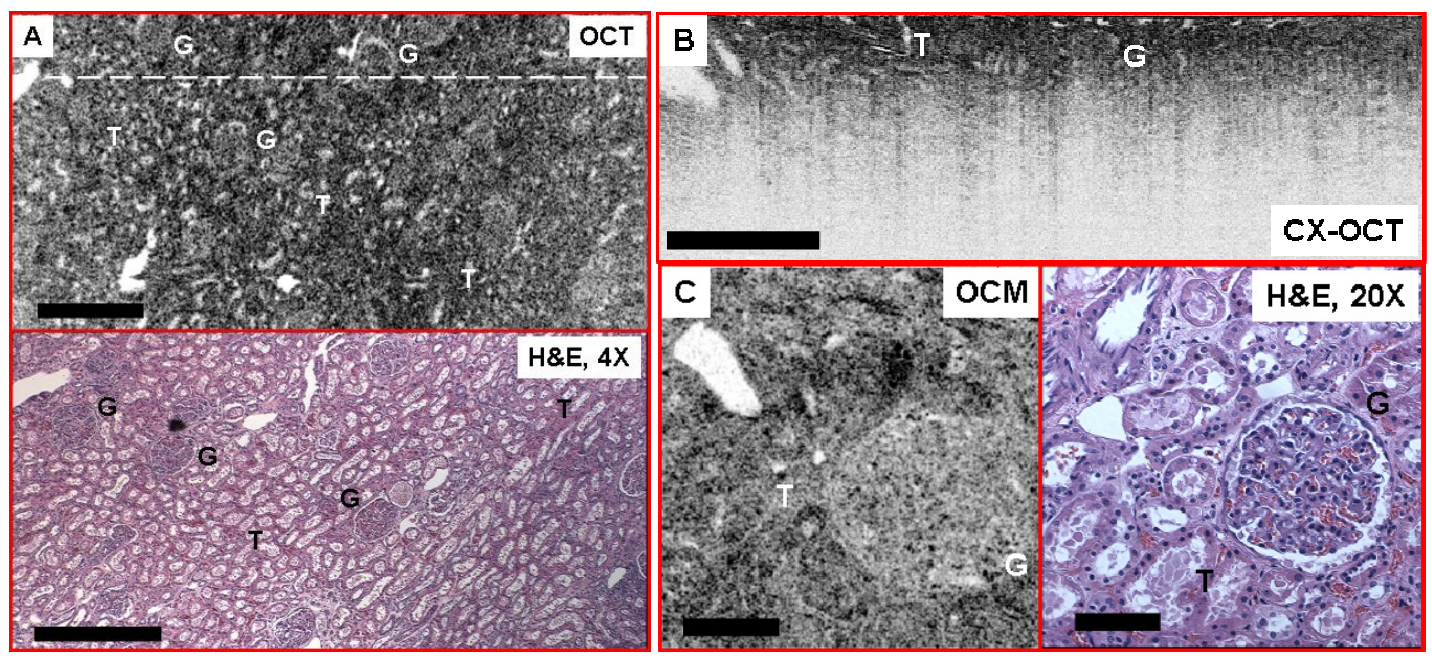

Figure 5.10. Normal human renal cortex. (A). En face OCT image and corresponding H\&E histologic section demonstrates architectural features of normal cortex, including glomeruli (G) and convoluted tubules (T). Scale bar: 500 um. (B) Cross-sectional OCT (CX-OCT) image corresponding to the scanning position delineated as a white line in (A). Scale bars: 500 um. (C) OCM image and corresponding H\&E histologic section demonstrates in more detail a glomerulus with surrounding convoluted tubules. The complex network of glomerular capillaries is represented by round pale regions in the OCM images. Scale bar $100 \mathrm{um}$. 
The normal kidney medulla is characterized by collecting tubules and loops of Henle, as shown in Figure 5.11. The en face OCT image in Fig. 5.11A shows a region rich of round hyposcattering tubules, correlating well with the histologic section. Fig. 5.11B shows the cross-section OCT image corresponding to the scanning position noted in Fig. 5.11A. Compared with en face OCT image, long and straight hyposcattering tubular structures are observed elongating into regions beneath the surface. The imaging depth of normal kidney is around $1 \mathrm{~mm}$ (Fig. 5.10B and Fig. 5.11B). This highlights the complimentary functions between en face and cross-sectional OCT images in assessing the specimen. Fig. 5.11C shows the OCM image of the medulla with corresponding histologic section. The distribution and morphology of these tublues can be observed more clearly under higher magnification. Tubular structures are characterized by hyposcattering regions surrounded by hyperscattering stroma. These preliminary results suggest OCT/OCM images can be used to identify the functioning units in both the cortex and medulla of normal human kidney.
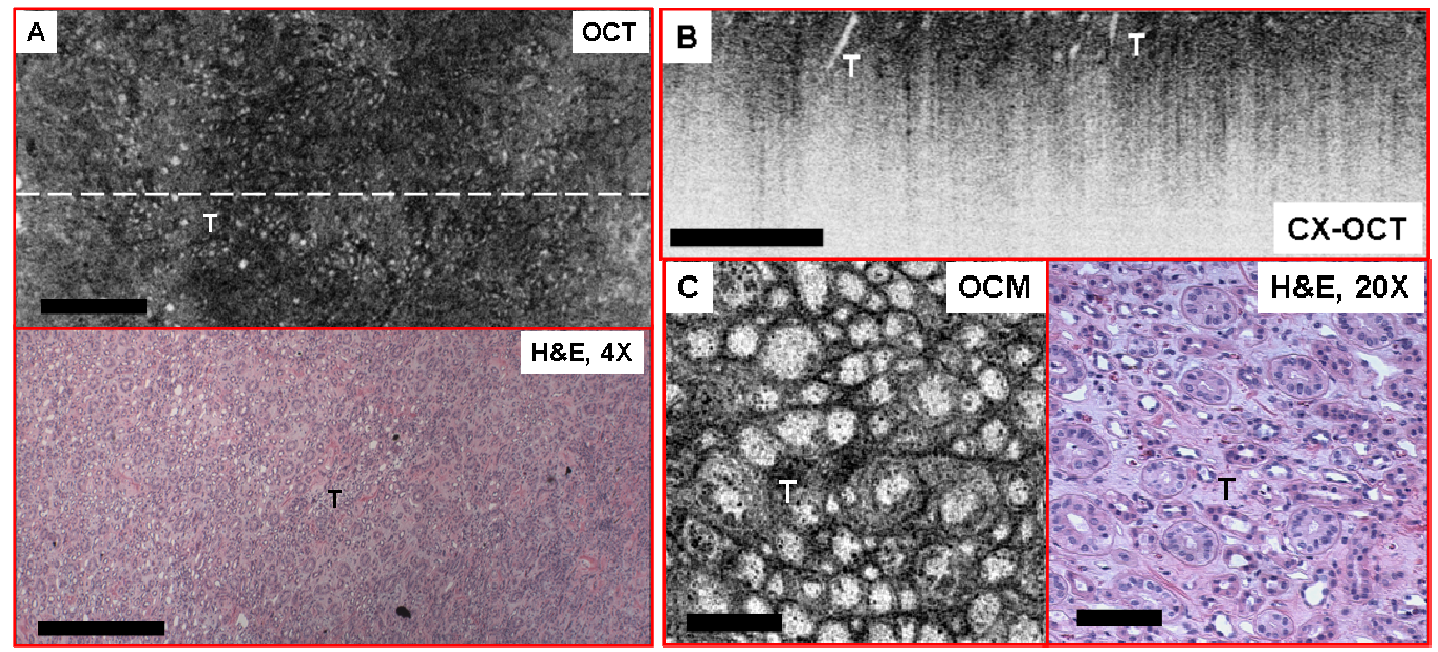

Figure 5.11. Normal human renal medulla. (A). En face OCT image and corresponding H\&E histologic section. (B) Cross-sectional OCT (CX-OCT) image, corresponding to the scanning position delineated as a white line in (A), demonstrates long, straight tubule structures $(T)$ elongating beneath the tissue surface. Scale bar: 500 um. (C) OCM image and corresponding H\&E histologic section shows architectural features of medullary structures (T) corresponding to collecting tubules and loops of Henle Sale bar: 100 um.

\section{References}

[1] D. Huang, E. A. Swanson, C. P. Lin, J. S. Schuman, W. G. Stinson, W. Chang, M. R. Hee, T. Flotte, K. Gregory, C. A. Puliafito, and J. G. Fujimoto, "Optical Coherence Tomography," Science, vol. 254, pp. 1178-1181, 1991.

[2] W. Drexler, U. Morgner, R. K. Ghanta, F. X. Kärtner, J. S. Schuman, and J. G. Fujimoto, "Ultrahigh-resolution ophthalmic optical coherence tomography," Nature Medicine, vol. 7, pp. 502507, 2001.

[3] J. G. Fujimoto, "Optical coherence tomography for ultrahigh resolution in vivo imaging," Nature Biotechnology, vol. 21, pp. 1361-1367, 2003.

[4] J. A. Izatt, M. R. Hee, G. M. Owen, E. A. Swanson, and J. G. Fujimoto, "Optical coherence microscopy in scattering media," Optics Letters, vol. 19, pp. 590-2, 1994/04/15 1994.

[5] J. A. Izatt, M. D. Kulkarni, H.-W. Wang, K. Kobayashi, and M. V. Sivak, Jr., "Optical coherence tomography and microscopy in gastrointestinal tissues," IEEE Journal of Selected Topics in Quantum Electronics, vol. 2, pp. 1017-28, 1996/12/ 1996.

[6] M. Kempe, W. Rudolph, and E. Welsch, "Comparative study of confocal and heterodyne microscopy for imaging through scattering media," Journal of the Optical Society of America aOptics Image Science and Vision, vol. 13, pp. 46-52, 1996. 
[7] A. D. Aguirre, Y. Chen, B. Bryan, H. Mashimo, Q. Huang, J. L. Connolly, and J. G. Fujimoto, "Cellular Resolution Ex Vivo Imaging of Gastrointestinal Tissues with Optical Coherence Microscopy," Endoscopy, vol. submitted, 2008.

[8] S. Bourquin, A. D. Aguirre, I. Hartl, P. Hsiung, T. H. Ko, J. G. Fujimoto, T. A. Birks, W. J. Wadsworth, U. Bunting, and D. Kopf, "Ultrahigh resolution real time OCT imaging using a compact femtosecond Nd : Glass laser and nonlinear fiber," Optics Express, vol. 11, pp. 32903297, 2003.

[9] A. D. Aguirre, N. Nishizawa, J. G. Fujimoto, W. Seitz, M. Lederer, and D. Kopf, "Continuum generation in a novel photonic crystal fiber for ultrahigh resolution optical coherence tomography at $800 \mathrm{~nm}$ and $1300 \mathrm{~nm}$," Optics Express, vol. 14, pp. 1145-1160, 2006.

[10] S. A. Hundahl, I. D. Fleming, A. M. Fremgen, and H. R. Menck, "A National Cancer Data Base Report on 53,856 Cases of Thyroid Carcinoma Treated in the US, 1985-1995," Cancer, vol. 83, pp. 2638-2648, 1998.

[11] "Cancer Facts and Figures," American Cancer Society 2009.

[12] P. W. Wiest, M. F. Hartshorne, P. D. Inskip, L. A. Crooks, B. S. Vela, R. J. Telepak, M. R. Williamson, R. Blumhardt, J. M. Bauman, and M. Tekkel, "Thyroid palpation versus highresolution thyroid ultrasonography in the detection of nodules," Journal of Ultrasound in Medicine, vol. 17, pp. 487-496, 1998.

[13] B. A. Carroll, "Asymptomatic Thyroid-Nodules - Incidental Sonographic Detection," American Journal of Roentgenology, vol. 138, pp. 499-501, 1982.

[14] A. Brander, P. Viikinkoski, J. Nickels, and L. Kivisaari, "Thyroid-Gland - Ultrasound Screening in a Random Adult-Population," Radiology, vol. 181, pp. 683-687, Dec 1991.

[15] J. N. Bruneton, C. Balumaestro, P. Y. Marcy, P. Melia, and M. Y. Mourou, "Very High-Frequency (13 Mhz) Ultrasonographic Examination of the Normal Neck - Detection of Normal Lymph-Nodes and Thyroid-Nodules," Journal of Ultrasound in Medicine, vol. 13, pp. 87-90, 1994.

[16] C. Zhou, Y. H. Wang, A. D. Aguirre, T.-H. Tsai, J. L. Connolly, and J. G. Fujimoto, "Ex vivo Imaging of Human Thyroid Pathology Using Integrated Optical Coherence Tomography (OCT) and Optical Coherence Microscopy (OCM)," vol. In review, 2009.

[17] "Cancer Facts and Figures," American Cancer Society 2010.

[18] M. J. Silverstein, A. Recht, M. D. Lagios, I. J. Bleiweiss, P. W. Blumencranz, T. Gizienski, S. E. Harms, J. Harness, R. J. Jackman, V. S. Klimberg, R. Kuske, G. M. Levine, M. N. Linver, E. A. Rafferty, H. Rugo, K. Schilling, D. Tripathy, F. A. Vicini, P. W. Whitworth, and S. C. Willey, "Imagedetected breast cancer: state-of-the-art diagnosis and treatment (vol 209, pg 504, 2009)," Journal of the American College of Surgeons, vol. 209, pp. 802-802, 2009.

[19] S. H. Parker, F. Burbank, R. J. Jackman, C. J. Aucreman, G. Cardenosa, T. M. Cink, J. L. Coscia, G. W. Eklund, W. P. Evans, P. R. Garver, H. F. Gramm, D. K. Haas, K. M. Jacob, K. M. Kelly, L. K. Killebrew, M. C. Lechner, S. J. Perlman, A. P. Smid, L. Tabar, F. E. Taber, and R. T. Wynn, "Percutaneous Large-Core Breast Biopsy - a Multiinstitutional Study," Radiology, vol. 193, pp. 359-364, 1994.

[20] J. P. Crowe, A. Rim, R. J. Patrick, L. A. Rybicki, S. F. Grundfest-Broniatowski, J. A. Kim, and K. B. Lee, "Does core needle breast biopsy accurately reflect breast pathology?," Surgery, vol. 134, pp. 523-526, 2003.

[21] M. F. Dillon, A. D. K. Hill, C. M. Quinn, A. O'Doherty, E. W. McDermott, and N. O'Higgins, "The accuracy of ultrasound, stereotactic, and clinical core biopsies in the diagnosis of breast cancer, with an analysis of false-negative cases," Annals of Surgery, vol. 242, pp. 701-707, 2005.

[22] V. I. Shah, U. Raju, D. Chitale, V. Deshpande, N. Gregory, and V. Strand, "False-negative core needle biopsies of the breast - An analysis of clinical, radiologic, and pathologic findings in 27 consecutive cases of missed breast cancer," Cancer, vol. 97, pp. 1824-1831, 152003.

[23] T. Agarwal, B. Patel, P. Rajan, D. A. Cunningham, A. Darzi, and D. J. Hadjiminas, "Core biopsy versus FNAC for palpable breast cancers. Is image guidance necessary?," European Journal of Cancer, vol. 39, pp. 52-56, 2003.

[24] R. J. Jackman, K. W. Nowels, J. Rodriguez-Soto, F. A. Marzoni, S. I. Finkelstein, and M. J. Shepard, "Stereotactic, automated, large-core needle biopsy of nonpalpable breast lesions: False-negative and histologic underestimation rates after long-term follow-up," Radiology, vol. 210, pp. 799-805, 1999. 
[25] S. H. Parker, W. E. Jobe, M. A. Dennis, A. T. Stavros, K. K. Johnson, W. F. Yakes, J. E. Truell, J. G. Price, A. B. Kortz, and D. G. Clark, "Us-Guided Automated Large-Core Breast Biopsy," Radiology, vol. 187, pp. 507-511, 1993.

[26] L. Liberman, T. L. Feng, D. D. Dershaw, E. A. Morris, and A. F. Abramson, "US-guided core breast biopsy: Use and cost-effectiveness," Radiology, vol. 208, pp. 717-723, 1998.

[27] J. L. Connolly, J. Boyages, A. J. Nixon, G. Peiro, I. Gage, B. Silver, A. Recht, J. R. Harris, and S. $J$. Schnitt, "Predictors of breast recurrence after conservative surgery and radiation therapy for invasive breast cancer," Modern Pathology, vol. 11, pp. 134-139, 1998.

[28] I. Gage, S. J. Schnitt, A. J. Nixon, B. Silver, A. Recht, S. L. Troyan, T. Eberlein, S. M. Love, R. Gelman, J. R. Harris, and J. L. Connolly, "Pathologic margin involvement and the risk of recurrence in patients treated with breast-conserving therapy," Cancer, vol. 78, pp. 1921-1928, 1996.

[29] C. C. Park, M. Mitsumori, A. Nixon, A. Recht, J. Connolly, R. Gelman, B. Silver, S. Hetelekidis, A. Abner, J. R. Harris, and S. J. Schnitt, "Outcome at 8 years after breast-conserving surgery and radiation therapy for invasive breast cancer: Influence of margin status and systemic therapy on local recurrence," Journal of Clinical Oncology, vol. 18, pp. 1668-1675, 2000.

[30] F. J. Fleming, A. D. K. Hill, E. W. Mc Dermott, A. O'Doherty, N. J. O'Higgins, and C. M. Quinn, "Intraoperative margin assessment and re-excision rate in breast conserving surgery," European Journal of Surgical Oncology, vol. 30, pp. 233-237, 2004.

[31] G. P. Swanson, K. Rynearson, and R. Symmonds, "Significance of margins of excision on breast cancer recurrence," American Journal of Clinical Oncology-Cancer Clinical Trials, vol. 25, pp. 438-441, Oct 2002.

[32] D. Krag, D. Weaver, T. Ashikaga, F. Moffat, V. S. Klimberg, C. Shriver, S. Feldman, R. Kusminsky, M. Gadd, J. Kuhn, S. Harlow, and P. Beitsch, "The sentinel node in breast cancer - A multicenter validation study," New England Journal of Medicine, vol. 339, pp. 941-946, 1998.

[33] R. E. Mansel, L. Fallowfield, M. Kissin, A. Goyal, R. G. Newcombe, J. M. Dixon, C. Yiangou, K. Horgan, N. Bundred, I. Monypenny, D. England, M. Sibbering, T. J. Abdullah, L. Barr, U. Chetty, D. H. Sinnett, A. Fleissig, D. Clarke, and P. J. Ell, "Randomized multicenter trial of sentinel node biopsy versus standard axillary treatment in operable breast cancer: The ALMANAC trial," Journal of the National Cancer Institute, vol. 98, pp. 599-609, 2006.

[34] P. Schrenk, R. Rieger, A. Shamiyeh, and W. Wayand, "Morbidity following sentinel lymph node biopsy versus axillary lymph node dissection for patients with breast carcinoma," Cancer, vol. 88, pp. 608-614, 2000.

[35] T. P. Olson, J. Harter, A. Munoz, D. M. Mahvi, and T. M. Breslin, "Frozen section analysis for intraoperative margin assessment during breast-conserving surgery results in low rates of reexcision and local recurrence," Annals of Surgical Oncology, vol. 14, pp. 2953-2960, 2007.

[36] S. A. McLaughlin, L. M. Ochoa-Frongia, S. M. Patil, H. S. Cody, and L. M. Sclafani, "Influence of frozen-section analysis of sentinel lymph node and lumpectomy margin status on reoperation rates in patients undergoing breast-conservation therapy," Journal of the American College of Surgeons, vol. 206, pp. 76-82, 2008.

[37] A. D. Aguirre, Y. Chen, B. Bryan, H. Mashimo, Q. Huang, J. L. Connolly, and J. G. Fujimoto, "Cellular resolution ex vivo imaging of gastrointestinal tissues with optical coherence microscopy," J Biomed Opt, vol. 15, p. 016025, 2010.

[38] C. Zhou, Y. Wang, A. D. Aguirre, T.-H. Tsai, D. W. Cohen, J. L. Connolly, and J. G. Fujimoto, "Ex vivo imaging of human thyroid pathology using integrated optical coherence tomography and optical coherence microscopy," J Biomed Opt, vol. 15, p. 016001, 2010.

[39] S. Daimon and I. Koni, "Glomerular enlargement in the progression of mesangial proliferative glomerulonephritis," Clinical Nephrology, vol. 49, pp. 145-152, 1998. 


\title{
6. Functional Brain Imaging with Optical Coherence Tomography Sponsors
}

\section{Sponsors}

Air Force Office of Scientific Research - FA9550-07-1-0101, FA9550-07-1-0014

National Institutes of Health - 5R01-NS057476-02, 5R01-CA75289-13, R01-EY11289-24

\author{
Project Staff \\ Prof. James G. Fujimoto, Dr. Chao Zhou (MIT) \\ Prof. David A. Boas, Dr. Vivek J. Srinivasan, Dr. Sava Sakadzic, Svetlana Ruvinskaya (MGH)
}

Quantitative and localized measurements of cerebral blood flow (CBF) are important for developing an understanding of major cerebrovascular diseases. For example, alterations in CBF may contribute to the pathogenesis of stroke and Alzheimer's disease [1]. Rat and mouse models are important in preclinical cerebrovascular research. However, there is no existing method for in vivo determination of absolute CBF values with high resolution in small animals. Magnetic resonance imaging (MRI) [2] and positron emission tomography (PET) [3] based methods of flow measurements provide spatial maps of CBF, but they are limited in their temporal and spatial resolution (resolution is $\sim 2-3 \mathrm{~mm}$ with MRI and $\sim 7-10 \mathrm{~mm}$ with PET) . Among optical methods, two-photon fluorescence microscopy can characterize both transverse velocity and linear density of red blood cells [4]; however, the determination of CBF requires knowledge of the flow profile as well as vessel orientation, which is difficult to obtain using this technique. Laser speckle imaging can provide high spatiotemporal resolution, but this method does not offer depth specificity [5]. The gold standard for determining regional blood flow is autoradiography which provides threedimensional spatial information, but contains no information about the temporal evolution of CBF changes [6]. Doppler OCT overcomes many of the limitations of the aforementioned techniques by using path delay of light to gate out multiply scattered light [7-10]. OCT enables measurement of average velocity over well-defined volumes, determined by the numerical aperture in the transverse direction and by the coherence length in the axial direction.

Working in collaboration with Dr. David Boas at the Martinos Center for Biomedical Imaging of the Massachusetts General Hospital and Harvard Medical School, we are investigating the use of Doppler OCT for CBF measurements. We have developed a quantitative statistical model for static tissue and validated this model in a scattering phantom. Using this model to guide the signal processing, we have performed 1) quantitative, absolute flow measurements in the rat cortex and 2) achieved comprehensive visualization of the cortical vasculature down the capillary level [11]. We have measured flow values comparable to those obtained in rats under similar anesthetic conditions using autoradiography.

A spectral domain OCT based microscope for in vivo imaging of the rat cerebral cortex was designed for this study. The system employs a superluminescent diode with a center wavelength of $856 \mathrm{~nm}$ and bandwidth of $54 \mathrm{~nm}$. The axial (depth) resolution in the tissue is $4.8 \mathrm{um}$ and the imaging speed is $22 \mathrm{kHz}$ axial scan rate. For flow measurements and angiography, transverse scanning speeds of $21 \mathrm{~mm} / \mathrm{s}$ and $0.5 \mathrm{~mm} / \mathrm{s}$ were chosen respectively due to the different requirements of these two imaging modes. Fig. 6.1A shows an OCT angiogram of the rat cortex. The transverse resolution is $12 \mathrm{um}$. Fig. 6.1B shows a zoomed view of an arterial anasthmosis. Fig. 6.1C shows an angiogram obtained with a 3.6um resolution. The results of absolute blood flow measurements in the rat cortex are shown in Figure 6.2. Venulus are marked and numbered on the OCT angiogram (Fig. 6.2A). Flow measurements at each location along with standard errors over 10 repeated measurements are shown in Fig. 6.2B.

Since flow measurements are performed by scanning an optical beam over the cortex, the most natural units for the Doppler OCT flow measurements are volume per unit cortical surface per unit time. The measurements yielded $1.07+/-0.16 \times 10^{-3} \mathrm{~mL} / \mathrm{mm}^{2} / \mathrm{min}$. This result is consistent with a recent iodo $\left[{ }^{14} \mathrm{C}\right]$ antipyrine autoradiographic study which measured resting $\mathrm{CBF}$ in the somatosensory cortex [12]. Moreover, due to the advanced signal processing methods used it in this study, it is possible to visualize capillaries with sizes at or below the optical resolution. 

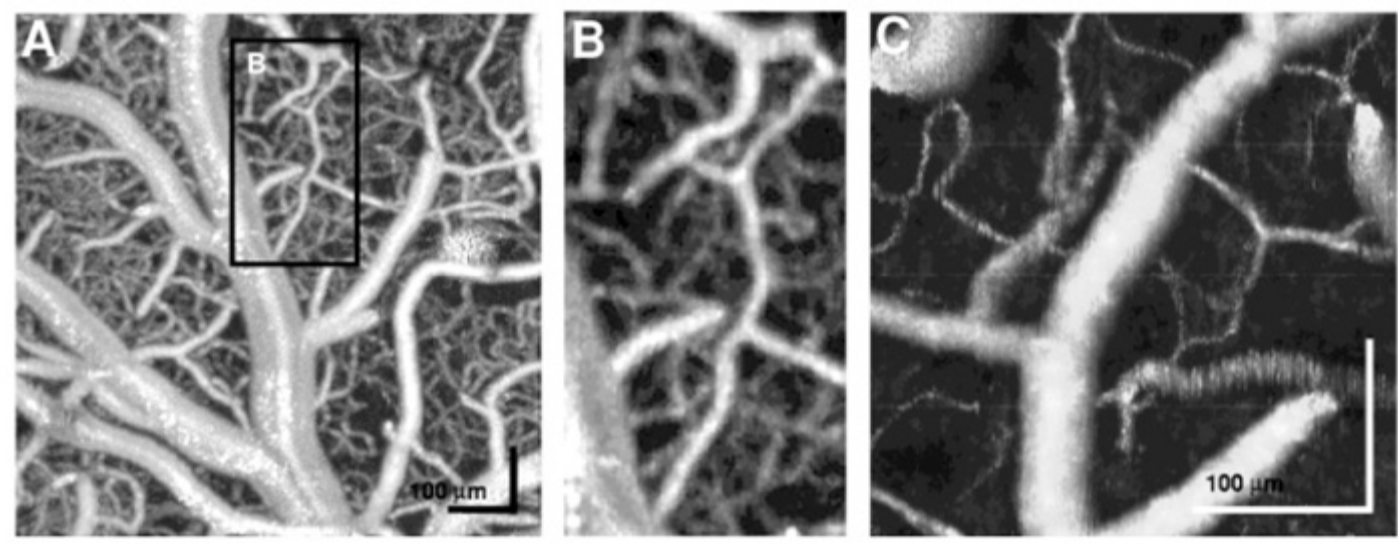

Figure 6.1. (A) OCT maximum intensity projection (MIP) angiogram of the rat somatosensory cortex showing comprehensive visualization of the vasculature at $12 \mathrm{um}$ transverse resolution. (B) zoom of arterial anasthmosis, showing visualization of surface vessels as well as capillaries below. (C) OCT MIP angiogram acquired with a 3.6 um transverse resolution in a different animal. The transverse scanning speed was chosen to yield identical temporal power spectral densities at both resolutions. The apparent diameter of capillaries is increased as the transverse resolution is increased. In addition, due to the smaller depth of field, fewer vessels are visualized in the high transverse resolution MIP angiogram (C).
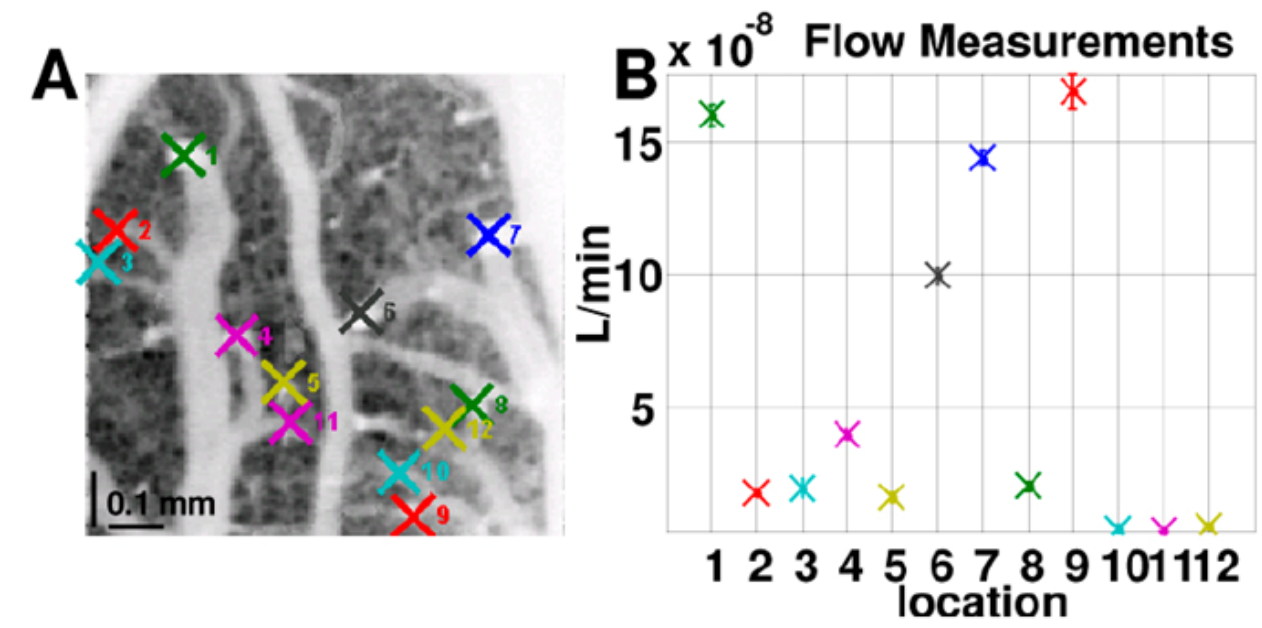

Figure 6.2. Absolute values of flow measurements in the rat cortex in ascending venules. $(A)$ Locations for flow measurements and $(B)$ absolute flow measurements at the designated locations. Standard errors over 10 repeated measurements are shown.

The measurements presented in the study have the advantage that they do not require exogenous contrast and can be directly compared longitudinally in a single animal over time or cross-sectionally across groups of animals. This capability should greatly aid studies of cerebral pathophysiology and aid in the testing of pharmacological agents in animal models.

\section{References}

[1] H. Girouard and C. ladecola, "Neurovascular coupling in the normal brain and in hypertension, stroke, and Alzheimer disease," J Appl Physiol, vol. 100, pp. 328-35, 2006.

[2] F. Calamante, D. L. Thomas, G. S. Pell, J. Wiersma, and R. Turner, "Measuring cerebral blood flow using magnetic resonance imaging techniques," J Cereb Blood Flow Metab, vol. 19, pp. 70135, 1999. 
[3] W. D. Heiss, R. Graf, K. Wienhard, J. Lottgen, R. Saito, T. Fujita, G. Rosner, and R. Wagner, "Dynamic penumbra demonstrated by sequential multitracer PET after middle cerebral artery occlusion in cats," J Cereb Blood Flow Metab, vol. 14, pp. 892-902, 1994.

[4] D. Kleinfeld, P. P. Mitra, F. Helmchen, and W. Denk, "Fluctuations and stimulus-induced changes in blood flow observed in individual capillaries in layers 2 through 4 of rat neocortex," Proc Natl Acad Sci U S A, vol. 95, pp. 15741-6, 1998.

[5] J. D. Briers and A. F. Fercher, "Retinal blood-flow visualization by means of laser speckle photography," Invest Ophthalmol Vis Sci, vol. 22, pp. 255-9, 1982.

[6] O. Sakurada, C. Kennedy, J. Jehle, J. D. Brown, G. L. Carbin, and L. Sokoloff, "Measurement of local cerebral blood flow with iodo [14C] antipyrine," Am J Physiol, vol. 234, pp. H59-66, 1978.

[7] Z. Chen, T. E. Milner, D. Dave, and J. S. Nelson, "Optical Doppler tomographic imaging of fluid flow velocity in highly scattering media," Opt Lett, vol. 22, pp. 64-6, 1997.

[8] J. A. Izatt, M. D. Kulkarni, S. Yazdanfar, J. K. Barton, and A. J. Welch, "In vivo bidirectional color Doppler flow imaging of picoliter blood volumes using optical coherence tomography," Opt Lett, vol. 22, pp. 1439-41, 1997.

[9] R. Leitgeb, L. Schmetterer, W. Drexler, A. Fercher, R. Zawadzki, and T. Bajraszewski, "Real-time assessment of retinal blood flow with ultrafast acquisition by color Doppler Fourier domain optical coherence tomography," Opt Express, vol. 11, pp. 3116-21, 2003.

[10] B. White, M. Pierce, N. Nassif, B. Cense, B. Park, G. Tearney, B. Bouma, T. Chen, and J. de Boer, "In vivo dynamic human retinal blood flow imaging using ultra-high-speed spectral domain optical coherence tomography," Opt Express, vol. 11, pp. 3490-7, 2003.

[11] V. J. Srinivasan, S. Sakadzic, I. Gorczynska, S. Ruvinskaya, W. Wu, J. G. Fujimoto, and D. A. Boas, "Quantitative cerebral blood flow with optical coherence tomography," Opt Express, vol. 18, pp. 2477-94, 2010.

[12] R. K. Wang and Z. Ma, "Real-time flow imaging by removing texture pattern artifacts in spectraldomain optical Doppler tomography," Opt Lett, vol. 31, pp. 3001-3, 2006. 


\section{Photothermal OCT for Molecular-Targeted Imaging}

\section{Sponsors}

National Institutes of Health - R01-CA75289-13

Air Force Office of Scientific Research FA9550-040-1-0011, FA9550-040-1-0046

\section{Project Staff}

Professor James G. Fujimoto, Dr. Chao Zhou, Hsiang-Chieh Lee, Tsung-Han Tsai (MIT)

Dr. Desmond Adler (LightLab Imaging)

Dr. David W. Cohen, M.D., Dr. Amy Mondelblatt, M.D., Dr. Yihong Wang, M.D., Dr. James L. Connolly, M.D. (BIDMC)

Optical coherence tomography (OCT) is a powerful tool for assessing tissue architectural morphology [1]. It enables three-dimensional imaging with resolutions approaching that of histopathology, but can be performed in vivo and in real-time without the need to remove and process specimens. Conventional OCT imaging is based on contrast from spatial variations in tissue scattering properties. The use of exogenous contrast agents would allow targeted imaging of specific cells, receptors or functional processes. OCT contrast enhancement has been demonstrated using scattering microspheres [2], iron oxide microparticles [3], and nanoparticles [4-8]. Gold nanoparticles are attractive for OCT due to their customizable absorption and scattering properties, biocompatibility, and ease of conjugation to antibodies and peptides that bind selectively with proteins associated with specific diseases [9]. Gold nanoparticles have also been used as effective photothermal therapy agents [10,11]. These characteristics offer by gold nanoparticles enable "three-in-one" [12] functionality as targeting probes, image contrast enhancers and therapeutic agents.

Our group has demonstrated phase-sensitive OCT to detect gold nanoshells as contrast agents in phantoms with high signal-to-noise ratio (SNR) [7]. The technique uses nanoshells with high absorption at $780 \mathrm{~nm}$, where tissue absorption is inherently low. An excitation laser at $808 \mathrm{~nm}$ induces small temperature gradients in sample regions that contain nanoparticles. These thermal variations modulate the sample's optical path length, which is detected using phase-sensitive OCT. Other groups working in parallel demonstrated photothermal OCT imaging in 3D cell constructs using immunotargeted gold nanospheres [8]. Photothermal OCT imaging is challenging in highly scattering tissues where multiple scattering plays an important role. In this study [13], we demonstrated the feasibility of photothermal OCT ex vivo in highly scattering human breast tissue.

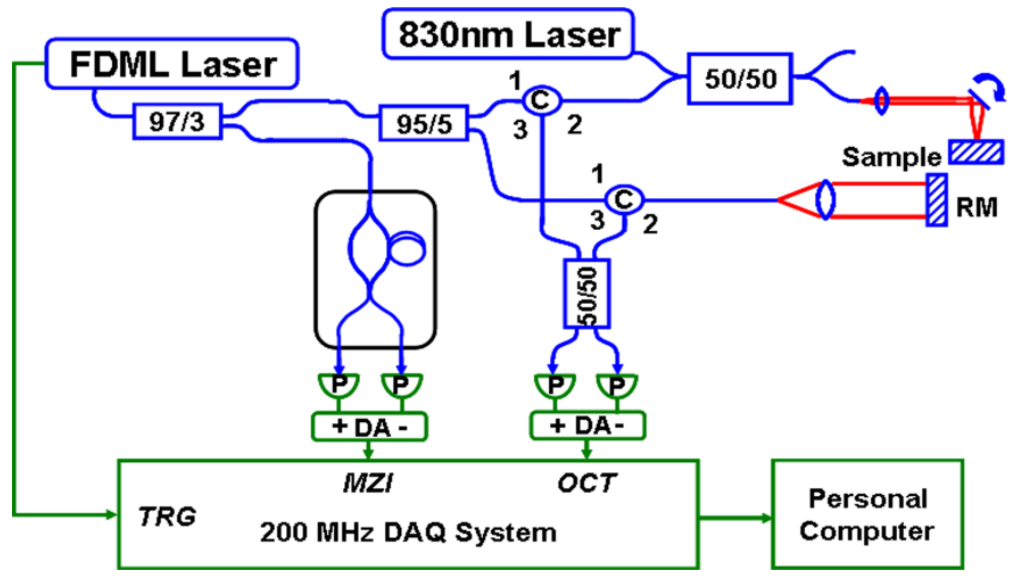

Figure 7.1. Schematic of photothermal OCT setup. A collinear photothermal excitation beam is coupled into a swept source OCT imaging system. Selective excitation occurs in volumes of the sample where gold nanoparticles are present. Phase modulation from temperature gradients is detected by the OCT. 


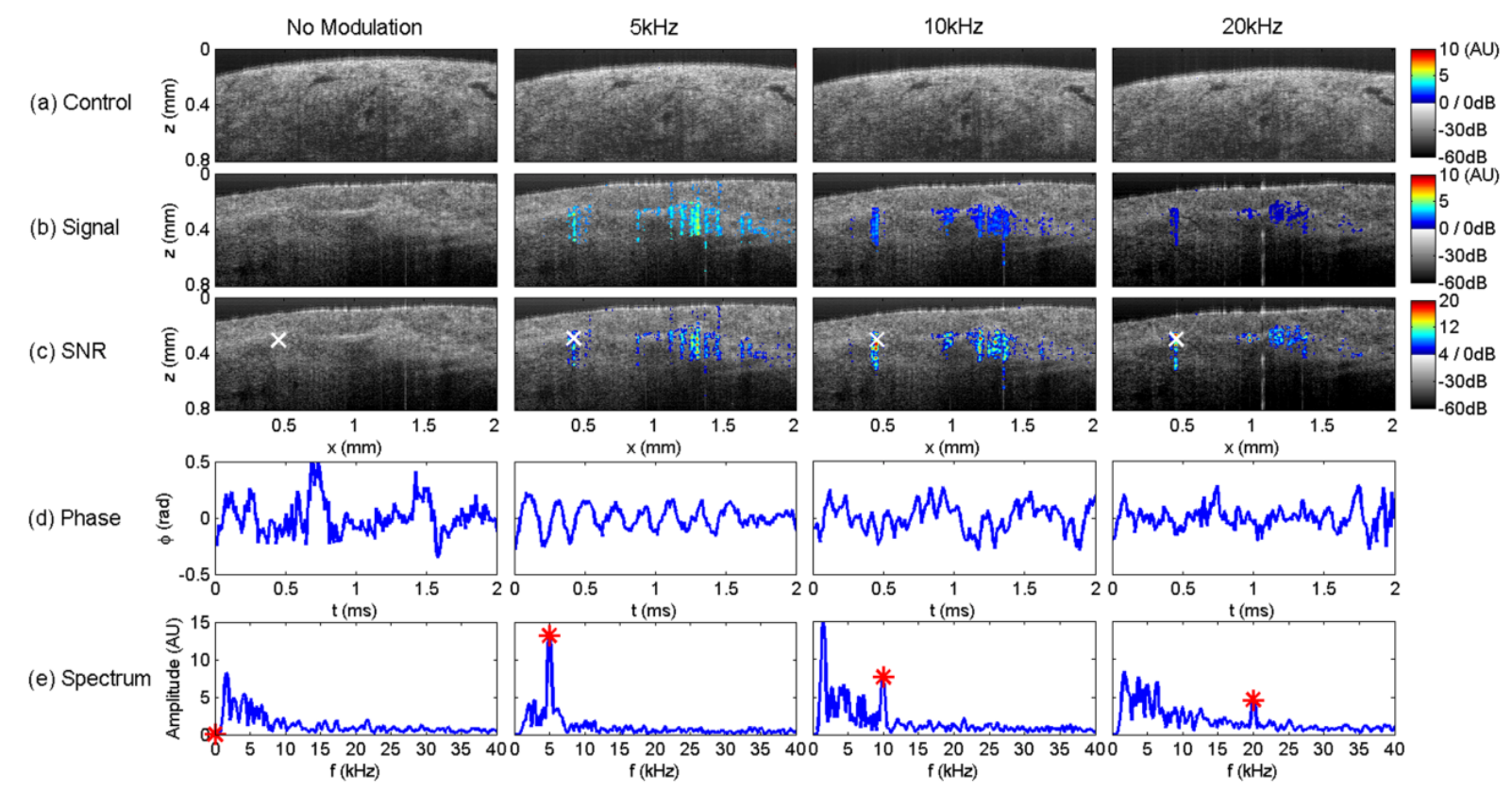

Figure 7.2. Photothermal OCT imaging in highly scattering human breast tissues ex vivo. (A) No photothermal signal is observed from control images in saline-injected specimen. Phase modulation signal (B) and SNR (C) images obtained from the nanoshell $\left(50 \mathrm{ul}, 5 \times 10^{9} \mathrm{ml}^{-1}\right)$ injected specimen at various photothermal modulation frequencies (no modulation, $5 \mathrm{kHz}, 10 \mathrm{kHz}$, and 20 $\mathrm{kHz}$ modulation) demonstrate localized photothermal signal. (D and E) Phase modulation time curves and frequency spectra corresponding to pixels marked in (C).

A $120 \mathrm{kHz}$ phase-sensitive SS-OCT system at $1300 \mathrm{~nm}$ was used to detect phase modulation induced by the $830 \mathrm{~nm}$ photothermal excitation of gold nanoshells (Figure 7.1). The photothermal modulation beam and the OCT imaging beam were coupled into the same optical fiber in order to maintain alignment during scanning. Localized phase modulations were successfully observed 300-600 um deep in scattering tissue by using an average excitation power of only $22 \mathrm{~mW}$ at modulation frequencies up to $20 \mathrm{kHz}$ (Figure 7.2). The peak-to-peak phase change is only $\sim 0.1 \mathrm{rad}$ for the $20 \mathrm{kHz}$ modulation (Fig. 7.2D), which corresponds to an optical path length change of $\sim 20 \mathrm{~nm}$. Assuming the signal was a result of accumulative effects from 50 um tissue above the detection point, the estimated peak-to-peak temperature change in tissue was only $\sim 1^{\circ} \mathrm{C}$. Signal to noise ratios SNR of 16, 24, and 23 were obtained for photothermal modulations at $5 \mathrm{kHz}, 10 \mathrm{kHz}$, and $20 \mathrm{kHz}$ (Fig. 7.2E), respectively. Localized phase modulation was also observed at a beam-scanning speed of $16 \mathrm{~mm} / \mathrm{s}$.

These results demonstrate that photothermal OCT is feasible in highly scattering tissues at low power levels consistent with those necessary for in vivo applications. In future studies, we will investigate antibody fragment or peptide marker conjugated nanoshells for molecularly targeted imaging.

\section{References}

[1] D. Huang, E. A. Swanson, C. P. Lin, J. S. Schuman, W. G. Stinson, W. Chang, M. R. Hee, T. Flotte, K. Gregory, C. A. Puliafito, and J. G. Fujimoto, "Optical Coherence Tomography," Science, vol. 254, pp. 1178-1181, 1991.

[2] T. M. Lee, A. L. Oldenburg, S. Sitafalwalla, D. L. Marks, W. Luo, F. J. J. Toublan, K. S. Suslick, and S. A. Boppart, "Engineered microsphere contrast agents for optical coherence tomography," Optics Letters, vol. 28, pp. 1546-1548, 2003.

[3] A. L. Oldenburg, J. R. Gunther, and S. A. Boppart, "Imaging magnetically labeled cells with magnetomotive optical coherence tomography," Optics Letters, vol. 30, pp. 747-9, 2005/04/01 2005. 
[4] H. Cang, T. Sun, Z. Y. Li, J. Y. Chen, B. J. Wiley, Y. N. Xia, and X. D. Li, "Gold nanocages as contrast agents for spectroscopic optical coherence tomography," Optics Letters, vol. 30, pp. 3048-3050, 2005.

[5] A. Agrawal, S. Huang, A. W. H. Lin, M. H. Lee, J. K. Barton, R. A. Drezek, and T. J. Pfefer, "Quantitative evaluation of optical coherence tomography signal enhancement with gold nanoshells," J Biomed Opt, vol. 11, p. 8, 2006.

[6] A. L. Oldenburg, M. N. Hansen, D. A. Zweifel, A. Wei, and S. A. Boppart, "Plasmon-resonant gold nanorods as low backscattering albedo contrast agents for optical coherence tomography," Optics Express, vol. 14, pp. 6724-6738, 2006.

[7] D. C. Adler, S. W. Huang, R. Huber, and J. G. Fujimoto, "Photothermal detection of gold nanoparticles using phase-sensitive optical coherence tomography," Optics Express, vol. 16, pp. 4376-4393, 2008.

[8] M. C. Skala, M. J. Crow, A. Wax, and J. A. Izatt, "Photothermal Optical Coherence Tomography of Epidermal Growth Factor Receptor in Live Cells Using Immunotargeted Gold Nanospheres," Nano Letters, vol. 8, pp. 3461-3467, 2008.

[9] K. Sokolov, M. Follen, J. Aaron, I. Pavlova, A. Malpica, R. Lotan, and R. Richards-Kortum, "Realtime vital optical imaging of precancer using anti-epidermal growth factor receptor antibodies conjugated to gold nanoparticles," Cancer research, vol. 63, pp. 1999-2004, 2003.

[10] D. P. O'Neal, L. R. Hirsch, N. J. Halas, J. D. Payne, and J. L. West, "Photo-thermal tumor ablation in mice using near infrared-absorbing nanoparticles," Cancer Letters, vol. 209, pp. 171-176, 25 2004.

[11] X. H. Huang, P. K. Jain, I. H. El-Sayed, and M. A. El-Sayed, "Gold nanoparticles: interesting optical properties and recent applications in cancer diagnostic and therapy," Nanomedicine, vol. 2, pp. 681-693, 2007.

[12] X. M. Yang, "Nano- and microparticle-based imaging of cardiovascular interventions: Overview," Radiology, vol. 243, pp. 340-347, 2007.

[13] C. Zhou, T. H. Tsai, D. C. Adler, H. C. Lee, D. W. Cohen, A. Mondelblatt, Y. H. Wang, J. L. Connolly, and J. G. Fujimoto, "Photothermal optical coherence tomography in ex vivo human breast tissues using gold nanoshells," Optics Letters, vol. 35, pp. 700-702, 2010. 


\section{Publication from 2009 to present}

1. D.C. Adler, C. Zhou, T.-H. Tsai, J. Schmitt, Q. Huang, H. Mashimo, and J.G. Fujimoto, "Threedimensional endomicroscopy of the human colon using optical coherence tomography," Opt. Exp. 17, 784-796, January 2009.

2. Y. Chen, A.D. Aguirre, L. Ruvinskaya, A. Devor, D.A. Boas, and J.G. Fujimoto, "Optical coherence tomography (OCT) reveals depth-resolved dynamics during functional brain activation," J. Neurosci. Methods, 178, 162-173, March 2009.

3. Y. Chen, L.N. Vuong, J. Liu, J. Ho, V.J. Srinivasan, I. Gorczynska, A.J. Witkin, J.S. Duker, J. Schuman, and J.G. Fujimoto, "Three-dimensional ultrahigh resolution optical coherence tomography imaging of age-related macular degeneration," Opt. Exp. 17, 4046-4060, March 2009.

4. H. Ishikawa, J. Kim, T.R. Friberg, G. Wollstein, L. Kagemann, M.L. Gabriele, K.A. Townsend, K.R. Sung, J.S. Duker, J.G. Fujimoto, and J.S. Schuman, "Three-dimensional optical coherence tomography (3D-OCT) image enhancement with segmentation-free contour modeling C-mode," Invest. Ophthalmol. and Vis. Sci. 50, 1344-1349, March 2009.

5. J.J. Kaluźny, M. Wojtkowski, B.L. Sikorski, M. Szkulmowski, A. Szkulmowska, T. Bajraszewski, J.G. Fujimoto, J.S. Duker, J.S. Schuman, and A. Kowalczyk, "Analysis of the outer retina reconstructed by high-resolution, three-dimensional spectral domain optical coherence tomography," Ophthalmic Surg. Lasers Imaging 40, 102-108 March/April 2009.

6. V.J. Srinivasan, Y. Chen, J.S. Duker, and J.G. Fujimoto, "In vivo functional imaging of intrinsic scattering changes in the human retina with high-speed ultrahigh resolution OCT," Opt. Exp. 17, 3861-3877, March 2009.

7. M. Wojtkowski, B.L. Sikorski, I. Gorczynska, M. Gora, M. Szkulmowski, D. Bukowska, J. Kaluzny, J.G. Fujimoto, and A. Kowalczyk, "Comparison of reflectivity maps and outer retinal topography in retinal disease by 3-D Fourier domain optical coherence tomography," Opt. Exp. 17, 4189-4207, March 2009.

8. I. Gorczynska, V.J. Srinivasan, L.N. Vuong, R.W.S. Chen, J.J. Liu, E. Reichel, M. Wojtkowski, J.S. Schuman, J.S. Duker, and J.G. Fujimoto, "Projection OCT fundus imaging for visualising outer retinal pathology in non-exudative age-related macular degeneration," Br. J. Ophthalmol. 93, 603-609, May 2009.

9. C. Zhou, D.C. Adler, L. Becker, Y. Chen, T.-H. Tsai, M. Figueirdo, J.M. Schmitt, J.G. Fujimoto, and H. Mashimo, "Effective treatment of chronic radiation proctitis using radiofrequency ablation," Ther. Adv. Gastroentrol. 2, 149-156, May 2009.

10. A.J. Witkin, L.N. Vuong, V.J. Srinivasan, I. Gorczynska, E. Reichel, C.R. Baumal, A.H. Rogers, J.S. Schuman, J.G. Fujimoto, and J.S. Duker, "High-speed ultrahigh resolution optical coherence tomography before and after ranibizumab for age-related macular degeneration," Ophthalmology 116, 956-963, May 2009.

11. K.R. Sung, G. Wollstein, R.A. Bilonick, K.A. Townsend, H. Ishikawa, L. Kagemann, R.J. Noecker, J.G. Fujimoto, and J.S. Schuman, "Effects of age on optical coherence tomography measurements of healthy retinal nerve fiber layer, macula, and optic nerve head," Ophthalmology 116, 1119-1124, June 2009.

12. J.S. Kim, H. Ishikawa, K.R. Sung, J. Xu, G. Wollstein, R.A. Bilonick, M.L. Gabriele, L. Kagemann, J.S. Duker, J.G. Fujimoto, and J.S. Schuman, "Retinal nerve fiber layer thickness measurement reproducibility improved with spectral domain optical coherence tomography," Br. J. Ophthalmol. 93, 1057-1063, August 2009.

13. D.C. Adler, C. Zhou, T.-H. Tsai, H.-C. Lee, L. Becker, J.M. Schmitt, Q. Huang, J.G. Fujimoto, and H. Mashimo, "Three-dimensional optical coherence tomography of Barrett's esophagus and buried glands beneath neo-squamous epithelium following radiofrequency ablation," Endoscopy 41, 773-776, September 2009.

14. J. Ho, A.C. Sull, L.N. Vuong, Y. Chen, J. Liu, J.G. Fujimoto, J.S. Schuman, and J.S. Duker, "Assessment of artifacts and reproducibility across spectral- and time-domain optical coherence tomography devices," Ophthalmology 116, 1960-1970, October 2009.

15. V.J. Srinivasan, S. Sakadzić, I. Gorczynska, S. Ruvinskaya, W. Wu, J.G. Fujimoto, and D. Boas, "Depth-resolved microscopy of cortical hemodynamics with optical coherence tomography," Opt. Lett. 34, 3086-3088, October 2009.

16. T.H. Tsai, C. Zhou, D.C. Adler, and J.G. Fujimoto, "Frequency comb swept lasers," Opt. Exp. 17, 21257-21270, November 2009. 
17. A.D. Aguirre, Y. Chen, B. Bryan, H. Mashimo, Q. Huang, J.L. Connolly, and J.G. Fujimoto, "Cellular resolution ex vivo imaging of gastrointestinal tissues with optical coherence microscopy," J. Biomed. Opt. 15, 016025-1-9, January/February 2010.

18. C. Zhou, Y. Wang, A.D. Aguirre, T.-H. Tsai, D.W. Cohen, J.L. Connolly, and J.G. Fujimoto, "Ex vivo imaging of human thyroid pathology using integrated optical coherence tomography and optical coherence microscopy," J. Biomed. Opt. 15, 016001-1—9, January/February 2010.

19. J.S. Kim, H. Ishikawa, M.L. Gabriele, G. Wollstein, R.A. Bilonick, L. Kagemann, J.G. Fujimoto, and J.S. Schuman, "Retinal nerve fiber layer thickness measurement comparability between time domain optical coherence tomography (OCT) and spectral domain OCT," Invest. Ophthalmol. and Vis. Sci., 51, 896-902, February 2010.

20. V.J. Srinivasan, S. Sakadzić, I. Gorczynska, S. Ruvinskaya, W. Wu, J.G. Fujimoto, and D. Boas, "Quantitative cerebral blood flow with optical coherence tomography," Opt. Exp. 18, 2477-2494, February 2010.

21. A.C. Sull, L.N. Vuong, L.L. Price, V.J. Srinivasan, I. Gorczynska, J.G. Fujimoto, J.S. Schuman, and J.S. Duker, "Comparison of spectral Fourier domain optical coherence tomography instruments for assessment of normal macular thickness," Retina 30, 235-245, February 2010.

22. C. Zhou, T.-H. Tsai, D.C. Adler, H.C. Lee, D.W. Cohen, A. Mondelblatt, Y. Wang, J.L. Connolly, and J.G. Fujimoto, "Photothermal optical coherence tomography in ex vivo human breast tissues using gold nanoshells," Opt. Lett. 35, 700-702, March 2010.

23. A.D. Aguirre, J. Sawinski, S.-W. Huang, C. Zhou, W. Denk, and J.G. Fujimoto, "High-speed optical coherence microscopy with autofocus adjustment and a miniaturized endoscopic imaging probe," Opt. Exp. 18, 4222-4239, March 2010.

24. D. Li, U. Demirbas, J.R. Birge, G.S. Petrich, L.A. Kolodziejski, A. Sennaroglu, F.X. Kärtner, and J.G. Fujimoto, "Diode-pumped passively mode-locked $\mathrm{GHz}$ femtosecond Cr:LiSAF laser with kW peak power," Opt. Lett. 35, 1446-1448, May 2010.

25. J. Ho, D.P. Castro, L.C. Castro, Y. Chen, J. Liu, C. Mattox, C. Krishnan, J.G. Fujimoto, J.S. Schuman, and J.S. Duker, "Clinical assessment of mirror artifacts in spectral domain optical coherence tomography," Invest. Ophthalmol. and Vis. Sci., forthcoming.

26. J. Ho, A.J. Witkin, Y. Chen, J. Liu, C. Baumal, A.H. Rogers, J.G. Fujimoto, J.S. Schuman, and J.S. Duker, "Volumetric analysis of subretinal and sub-retinal pigment epithelium pathologies via commercial spectral domain optical coherence tomography devices," Ophthalmology, forthcoming.

27. C.H. Judson, L.N. Vuong, I. Gorczynska, V. Srinivasan, J.G. Fujimoto, and J.S. Duker, "Intact retinal tissue and retinal pigment epithelium identified within a coloboma via high-speed, ultrahigh resolution optical coherence tomography," Retinal Cases Brief Rep., forthcoming.

28. A.D. Aguirre, D.C. Adler, and J.G. Fujimoto, "Practical guidelines for biological imaging with optical coherence tomography," submitted to Nat. Protocols.

29. B. Garita, M. Han, M.W. Jenkins, S.H. Seeholzer, C. Zhou, M. VanAuker, A.M. Rollins, M. Watanabe, J.G. Fujimoto, and K. Linask, "Optical coherence tomography (OCT) of the looping embryonic heart: II. Cardiac jelly composition and mechanosensing mechanisms," submitted to Circ. Res.

30. J. Ho, A.J. Witkin, J. Liu, Y. Chen, J.G. Fujimoto, and J.S. Schuman, "Documentation of intraretinal pigment epithelium migration via high speed ultrahigh resolution optical coherence tomography," submitted to Ophthalmology.

31. H. Ishikawa, G. Wollstein, L.A. Paunescu, S. Beaton, J.G. Fujimoto, and J.S. Schuman, "Detailed retinal layer segmentation with optical coherence tomography: A pilot study," submitted to Ophthalmology.

32. A. Li, C. Zhou, J. Moore, P. Zhang, T.-H. Tsai, H.-C. Lee, D. Romano, M. McKee, D. Schoenfeld, M. Serra, K. Raygor, H. Cantiello, J.G. Fujimoto, and R. Tanzi, "Changes in the expression of the Alzheimer's disease-associated presenilin gene in drosophilia heart leads to cardiac dysfunction," submitted to Hum. Mol. Genet.

33. B.K. Monson, V.J. Srinivasan, M.D. Wojtkowski, T.H. Ko, J.G. Fujimoto, J.S. Schuman, and J.S. Duker, "Ultrahigh resolution optical coherence tomography of an adult vitelliform macular dystrophy," submitted to Acta Ophthalmol. Scand.

34. K.R. Sung, G. Wollstein, H. Ishikawa, L. Kagemann, K.A. Townsend, J.G. Fujimoto, and J.S. Schuman, "Optical coherence tomography longitudinal assessment of retinal nerve fiber layer thickness in glaucoma using progression analysis," submitted to Ophthalmic Surg. Lasers Imaging. 
35. L.N. Vuong, V. Chaturredi, T.R. Hedges, III, E. Reichel, V.J. Srinivasan, I. Gorczynska, J.G. Fujimoto, and J.S. Duker, "Focal retinal outersegment gap defects identified by ultrahigh resolution optical coherence tomography," submitted to Ophthalmology.

36. G. Wollstein, L. Kagemann, R.A. Bilonick, H. Ishikawa, L.S. Folio, M.L. Gabriele, A.K. Ungar, J.S. Duker, J.G. Fujimoto, and J.S. Schuman, "Retinal nerve fiber layer and visual function loss in glaucoma: The tipping point," submitted to Am. J. Ophthalmol. 